\title{
Implicit Large Eddy Simulation of Weakly-Compressible Turbulent Channel Flow
}

\author{
I.W. Kokkinakis and D. Drikakis \\ Fluid Mechanics and Computational Science, Cranfield University, \\ Cranfield, MK43 0AL, United Kingdom
}

Corresponding author: Prof Dimitris Drikakis, Fluid Mechanics and Computational Science, Cranfield University, Cranfield, MK43 0AL, United Kingdom, d.drikakis@cranfield.ac.uk, Tel: +44-1234-754796 


\begin{abstract}
This paper concerns the accuracy of several high-resolution and high-order finite volume schemes in Implicit Large Eddy Simulation of weakly-compressible turbulent channel flow. The main objective is to investigate the properties of numerical schemes, originally designed for compressible flows, in low Mach compressible, near-wall turbulent flows. Variants of the Monotone Upstream-centred Scheme for Conservation Laws and Weighted Essentially NonOscillatory schemes for orders of accuracy ranging from second to ninth order, as well as with and without low Mach corrections, have been investigated. The performance of the schemes has been assessed against incompressible Direct Numerical Simulations. Detailed comparisons of the velocity profiles, turbulent shear stresses and higherorder turbulent statistics reveal that the low Mach correction can significantly reduce the numerical dissipation of the methods in low Mach boundary layer flows. The effects of the low Mach correction has more profound impact on second and third-order schemes, but they also improve the accuracy of fifth order schemes. The ninth-order Weighted Essentially Non-Oscillatory scheme is the least dissipative scheme and it is shown that the implementation of the low Mach correction in conjunction with this scheme has a significant anti-dissipative effect that adversely affects the accuracy. Finally, the computational cost required for obtaining the improved accuracy using increasingly higher order schemes is also discussed.
\end{abstract}

Keywords: Implicit Large Eddy Simulation (ILES), MUSCL, WENO, turbulent compressible channel flow, high-resolution, high-order, low Mach correction.

\title{
1. Introduction
}

Implicit Large-Eddy Simulations (ILES) originated from the observations made in [1] that the embedded dissipation of a certain class of numerical methods can be used in lieu of explicit sub-grid scale (SGS) models in classical Large-Eddy Simulation (LES) of turbulent flows. Modified equation analysis (MEA) was developed [2] in an effort to determine the stability of a difference equation by examining the truncation errors. The process begins from reducing a differential equation to a discretised equation by expanding each of its terms in a Taylor series. Such an analysis has been performed for the truncation error of certain schemes (e.g. [3-9]) leading to a better understanding of the implicit sub-grid dissipation.

In ILES, the Navier-Stokes equations (NSE) are discretised using high-resolution/high-order non-oscillatory methods without involving a low-pass filtering operation which gives rise to sub-grid scale (SGS) terms that require additional modelling. Instead, only the (implicit) de facto filtering introduced through the finite volume integration of the NSE over the grid cells is utilised in conjunction with non-linear numerical schemes that adhere to a number of principles; see [10, 11], and reviews $[8,9,12,13]$. It has been shown [3] that ILES methods need to be carefully designed, optimised, and validated for the particular differential equation to be solved. Direct MEA of high-resolution schemes for the Navier-Stokes equations is extremely difficult to be performed, thus understanding of the numerical properties of these methods to date still relies on performing computational experiments.

Several experimental studies [14-18] performed in the past to investigate the physics of turbulent boundary layers, established the turbulent channel flow test-case as one of the major 'canonical' flow problems to be used to perform detailed validation of numerical/computational methods [19-21]. A recent overview of the progress made regarding Direct Numerical Simulation (DNS) of wall-bounded turbulent flows with particular emphasis on channel and pipe flow geometries is given in [22-25] and references therein. Most of the DNS studies have used finite differences, Legendre polynomials and/or spectral methods based on Fourier representation or Chebychev-tau formulation. More recently, Discontinuous Galerkin (DG) methods have also been applied to DNS of turbulent channel flow [26, 27].

Incompressible DNS of fully developed channel flow has been published in [28-35]. These studies shed light on the turbulent flow physics, as well as provide data for the validation of numerical methods and turbulence models. A recent study [36] compared two fundamentally different DNS codes to assess the accuracy and reproducibility of standard and non-standard turbulence statistics, showing that the maximum relative deviations were below $0.2 \%$ for the mean flow, below $1 \%$ for the root-mean-square velocity, and pressure fluctuations, and below $2 \%$ for the three 
components of the turbulent dissipation. In comparison to incompressible DNS, there is only a limited number of compressible DNS studies and those have primarily been conducted for supersonic flows [37-40].

In $[41,42]$ the obtained DNS data were compared against experimental results, and then used to further probe and shed light on the turbulent flow physics. Other studies tried to ascertain the differences between channel and pipe turbulent flows through numerical computations [43, 44] and experiments [45, 46]. In [45] a comparison of experimental data with well-documented high Reynolds number $\left(R e_{\tau}=934\right)$ DNS [31] was presented. An excellent agreement for the streamwise velocity statistics between the two data sets was reported. Although the energy spectra were very similar, the DNS predicted a lower energy value in the logarithmic region, possibly due to the (shorter) dimension of the DNS box. The high computational cost required to successfully resolve all turbulent length-scales limits the applicability of DNS to relatively low Reynolds numbers and the incompressible Navier-Stokes equations. Note that DNS should be used (cautiously) as a benchmark rather than validation data. As a simulation result must ideally contain some assessment of the numerical errors and an error bar; however, this is not the case in the literature.

There are several research studies concerning classical LES of turbulent channel flow. Previous studies [47-50] (and references therein) have dealt with the development of SGS models; error contributions from SGS modelling and numerical schemes [19, 51-54]; error control through explicit filtering [53, 55, 56]; and the effects of different filtering procedures $[20,57,58]$.

Recent developments of explicit SGS models include the approximate deconvolution model (ADM) [47] which is an approximation of the non-filtered field by means of a truncated series expansion of the inverse filter operator. For an incompressible channel flow, ADM compared well against DNS data and showed a significant improvement [48] over the results obtained from typical SGS models such as the classical and dynamic Smagorinsky model. An evolution of the ADM is the adaptive local deconvolution model (ALDM) [49]. The ALDM is based on a non-linear discretization scheme, which contains several free deconvolution parameters that allow control of the truncation error. The free parameters are constrained such that the numerical viscosity optimally matches the theoretical eddy viscosity predicted by the analytical theories of turbulence and is therefore regarded as an ILES approach (in the broader context) compared to the ADM. The ALDM was applied to incompressible, turbulent channel flow to analyze its implicit SGS modelling capability in wall-bounded turbulence [50]. The simulations showed that the ALDM gives better results than the dynamic Smagorinsky model at the same grid resolution.

In the framework of classical LES, the accuracy of the SGS model is strongly influenced by the numerical contamination of the smallest resolved turbulent structures near the filter cut-off length [51, 52, 59]. Furthermore, it was found that the numerical error and SGS model interact with each other [19, 52-54]. It was reported [19] that for low-order finite-difference schemes, the truncation errors can exceed in magnitude the contribution of the SGS term. High-order numerical schemes are thus important in resolving the large energy-containing scales more accurately. However, they can also lead to contamination of the smallest resolved scales by truncation errors, in particular when using non-spectral methods. It was shown [56] that these errors can be controlled using an explicit filter. Nonetheless, mesh refinement still improved the results at a faster rate than the explicit filter size. Furthermore, previous studies [53] have shown that a minimum ratio of explicit filter-width to cell-size is necessary to be defined in order to prevent numerical errors from becoming larger than the contribution of the SGS turbulence closure terms and consequently saturating the solution. It was demonstrated that when the numerical simulations are carried out by a fourth-order finite-difference scheme, a filter width of at least twice the cell size should be used, whereas for a second-order scheme the filter width should be at least four times the cell size.

The influence of the numerical errors and SGS models in LES of channel flows, with and without explicit filtering were studied in [60]. When comparing to LES without explicit filtering, the difference in the mean velocity profiles was not large; however, the turbulence intensities were improved when explicit filtering was used. In [61], various dynamic SGS models were investigated to obtain the true filtered LES solution for an incompressible turbulent channel flow. It was hypothesized that the true LES solution should depend only on the filter width, regardless of the grid resolution. On the other hand, in ILES the solution converges towards DNS as the grid is refined because the filter width is implicitly and directly connected to the grid spacing. The effect of the different filtering methods was also examined in a subsequent study [57] showing that three-dimensional filtering gives better results than two-dimensional filtering. In [58], it was reported that the effect of filtering can be significant, with smooth filters increasing the total simulation error. Recently, [20] investigated the use of explicit filtering in LES for obtaining grid independent numerical solutions similar to the work of [61]. The convergence of the simulations was analysed for a turbulent channel flow at various friction Reynolds numbers $\left(R e_{\tau}=180,395\right.$, and 640), and it was shown that by using an 
explicit filter, the turbulent statistics and energy spectra became independent of mesh resolution. In [62], an accurate spectral LES approach was used to solve the incompressible, isothermal, Navier-Stokes equations. This allowed for simple, constant-coefficient Smagorinsky-type eddy viscosity SGS models without any wall damping functions to be used for modelling the decay of small scales. The approach termed "variational multiscale residual-based turbulence modelling" for LES has been further developed in $[63,64]$ showing very promising results.

Although LES is computationally less demanding than DNS, it still requires significant computational resources for simulating near wall turbulence at high Reynolds numbers. An alternative to LES is to make use of wall-layer models near the wall and use LES to resolve the outer region of the boundary layer, thus "relaxing" the grid resolution requirements near the wall. The wall-layer models can be broadly classified as: (i) equilibrium laws based on the logarithmic law, or some other assumed velocity profile (wall functions); (ii) zonal models, in which the turbulent boundary-layer equations (TBLE) are solved, weakly coupled to the outer-layer LES; and (iii) hybrid methods employing a Reynolds-Averaged Navier-Stokes (RANS)-based turbulence model near the wall and LES in the outer layer. A thorough review of the above is provided by Piomelli [65].

The best-known realisation of the hybrid framework is the Detached Eddy Simulation (DES) method by Spalart et al. [66]. In DES the interface location is dictated by the grid parameters through a switching condition. In [67] DES was used in the simulation of a turbulent channel flow. The results showed a non-physical buffer layer developing near the RANS/LES interface caused by the misalignment of the log layers between the RANS and LES regions. Due to the log-layer mismatch, the skin-friction coefficient was under-predicted by approximately $15 \%$. In the most commonly used DES implementation, the entire boundary layer is modelled by RANS [68, 69]. Using the $K-\epsilon$ model, $[68,69]$ carried out hybrid simulations of channel flow and introduced additional filtering at the interface to reduce the log-layer mismatch. Although these methods are promising, the amplitude of the stochastic forcing and the width of the additional filtering need both to be determined empirically. In [70] a stochastic backscatter model was applied to the wall-modeled DES of a channel flow showing improvements in the prediction of the mean velocity profile.

Other DES studies [71, 72] also reported issues in coupling the modeled and LES resolved regions, especially when more complex geometries and flows were considered in comparison to a plane flat surface [73-76]. More recently, a dynamic slip wall boundary condition for wall-modelled LES [77] was proposed, which gave encouraging results for separated flows over aerofoils. In [78], both ILES and the immersed-interface treatment of the wall boundaries showed to provide high computational efficiency on very coarse meshes for backward-facing step and periodic hill flows. Another category of near-wall models has been proposed by [79], which has been used in RANS, but may also prove promising for DES.

Although there is an extensive body of published research regarding the solution of turbulent channel flows using DNS, classical LES and DES, ILES investigations are still limited in number [80-83]. Previous research [80-83] has indicated that ILES is capable of reproducing first and second order statistical moments of the velocity field. Reviews examining the accuracy of ILES in other canonical problems such as the turbulence decay in a Taylor-Green vortex have also been published [84, 85]. Despite the above literature, there has been no systematic attempt to investigate the behaviour of different high-order compressible ILES methods in compressible turbulent channel flows. The aim of this study is to present a detailed investigation of the accuracy of a number of popular numerical schemes, originally designed for shock-capturing, with respect to weakly-compressible, turbulent channel flow. The specific objectives are: (i) to investigate the accuracy of the Monotone Upstream-centred Scheme for Conservation Laws (MUSCL) $2^{\text {nd }}$ to $5^{\text {th }}$, and the Weighted Essentially Non-Oscillatory (WENO) $5^{\text {th }}$ to $9^{\text {th }}$-order accurate slope limiter schemes against DNS data; (ii) to examine the effects of the low Mach correction of Thornber et al. [86, 87] on the accuracy of the MUSCL and WENO schemes; and (iii) to compare the numerical schemes with respect to their computational cost. The numerical assessment has been made using the incompressible DNS data of Moser et al. [30], which correspond to a friction Reynolds number of $R e_{\tau}=395$ (based on friction velocity $u_{\tau}$ ).

The paper is organised as follows: The governing equations and numerical schemes employed are briefly presented in $\S 2$. A description of the numerical set-up used in the simulation of the turbulent plane channel flow is given in $\S 3$, detailing the initial and boundary conditions, the implementation of the forcing term, and the statistical quantities utilised in the analysis of the results. $\$ 4$ presents the results from a series of ILES computations examining the accuracy and efficiency of different numerical schemes. Finally, the conclusions of the present study are summarised in $\S 5$. 


\section{Computational model}

\subsection{Governing equations}

The turbulent channel flow is governed by the Navier-Stokes equations, which in integral form are written as:

- Conservation of mass:

$$
\frac{\partial}{\partial t} \iiint_{V} \rho d V+\iint_{A} \rho \vec{u} \cdot \mathbf{d} \mathbf{A}=0,
$$

where $\mathbf{d A}=\vec{n} d A$ is the vector normal surface, $t$ is time, $\rho$ is the density and $\vec{u}$ is the velocity vector such that $\vec{u}=\left[u_{x}, u_{y}, u_{z}\right]^{T}=[u, v, w]^{T} . V$ and $A$ denote the volume and surface area of the cell, respectively.

- Conservation of momentum:

$$
\frac{\partial}{\partial t} \iiint_{V} \rho \vec{u} d V+\iint_{A}(\rho \vec{u}) \vec{u} \cdot \mathbf{d} \mathbf{A}=-\iint_{A} p \mathbf{d} \mathbf{A}+\iint_{A} \overline{\bar{\tau}} \cdot \mathbf{d} \mathbf{A}+\iiint_{V} \rho \overrightarrow{F_{b}} d V
$$

where $p$ is the static pressure, $\overline{\bar{\tau}}$ is the stress tensor and $\overrightarrow{F_{b}}$ denotes (external) body forces such as gravity.

The components of the stress tensor are given by:

$$
\overline{\bar{\tau}}=\left[\begin{array}{ccc}
\tau_{x x} & \tau_{x y} & \tau_{x z} \\
\tau_{y x} & \tau_{y y} & \tau_{y z} \\
\tau_{x z} & \tau_{z y} & \tau_{z z}
\end{array}\right]
$$

and the local stresses are defined by assuming the fluid is Newtonian:

$$
\tau_{i j}=\mu\left(\frac{\partial u_{i}}{\partial x_{j}}+\frac{\partial u_{j}}{\partial x_{i}}\right)-\frac{2}{3} \mu \nabla \vec{u} \delta_{i j}
$$

where $\delta_{i j}$ is the Kronecker delta with indices $(i),(j)=(x, y, z)$ and $\tau_{i j}=\tau_{j i}$ for $i \neq j$.

- Finally, conservation of the total energy for the control volume yields:

$$
\begin{aligned}
\frac{\partial}{\partial t} \iiint_{V} \rho E d V+\iint_{A} \rho E \vec{u} \cdot \mathbf{d} \mathbf{A}= & -\iint_{A} p \vec{u} \cdot \mathbf{d} \mathbf{A}+\iint_{A} k \nabla T \cdot \mathbf{d A} \\
& +\iint_{A}(\overline{\bar{\tau}} \cdot \vec{u}) \cdot \mathbf{d} \mathbf{A}+\iiint_{V} \rho \overrightarrow{F_{b}} \cdot \vec{u} d V
\end{aligned}
$$

where $E$ is the total energy per unit mass, $k$ is the thermal conductivity coefficient, and $T$ is the static temperature.

The continuity, momentum and total energy equations can also be written in a matrix form as:

$$
\frac{\partial}{\partial t} \iiint_{V} \vec{W} d V+\iint_{A}\left(\vec{F}_{c}-\vec{F}_{v}\right) d A=0
$$




$$
\vec{W}=\left[\begin{array}{c}
\rho \\
\rho u \\
\rho v \\
\rho w \\
\rho E
\end{array}\right], \quad \overrightarrow{F_{c}}=\left[\begin{array}{c}
\rho \vee \\
\rho u \vee+n_{x} p \\
\rho v \vee+n_{y} p \\
\rho w \vee+n_{z} p \\
\rho E \vee
\end{array}\right], \quad \overrightarrow{F_{v}}=\left[\begin{array}{c}
0 \\
n_{x} \tau_{x x}+n_{y} \tau_{x y}+n_{z} \tau_{x z} \\
n_{x} \tau_{y x}+n_{y} \tau_{y y}+n_{z} \tau_{y z} \\
n_{x} \tau_{z x}+n_{y} \tau_{z y}+n_{z} \tau_{z z} \\
n_{x} \Theta_{x}+n_{y} \Theta_{y}+n_{z} \Theta_{z}
\end{array}\right]
$$

where external forces have been neglected, and $\vee$ is the contravariant velocity given by:

$$
\vee=\vec{n} \cdot \vec{u}=n_{x} u+n_{y} v+n_{z} w
$$

and

$$
\begin{aligned}
& \Theta_{x}=u \tau_{x x}+v \tau_{x y}+w \tau_{x z}+k \frac{\partial T}{\partial x} \\
& \Theta_{y}=u \tau_{y x}+v \tau_{y y}+w \tau_{y z}+k \frac{\partial T}{\partial y} \\
& \Theta_{z}=u \tau_{z x}+v \tau_{z y}+w \tau_{z z}+k \frac{\partial T}{\partial z}
\end{aligned}
$$

The ideal gas law has been employed:

$$
p=\rho R T,
$$

where $R$ is the specific gas constant equal to $287 \mathrm{~J} \cdot \mathrm{kg} \cdot \mathrm{K}^{-1}$. The total energy per unit mass $(E)$ is the sum of internal energy $(e)$ plus kinetic and is computed by:

$$
E=e+\frac{1}{2}\left(u^{2}+v^{2}+w^{2}\right)
$$

where assuming a (calorically) perfect gas the internal energy $e$ is:

$$
e=c_{v} T=\frac{p}{\rho(\gamma-1)}
$$

$c_{v}$ denotes the specific heat capacity at constant volume, and $\gamma=1.4$ is the heat capacity ratio $\left(\gamma=c_{p} / c_{v}\right)$. The viscosity is approximated by the Sutherland's Law:

$$
\mu(T)=\mu_{0}\left(\frac{T}{T_{0}}\right)^{3 / 2} \frac{T_{0}+S_{u}}{T+S_{u}},
$$

where $S_{u}$ is the Sutherland temperature $(110.4 \mathrm{~K})$, while the values of the reference temperature and viscosity are $T_{0}=288.15 \mathrm{~K}$ and $\mu_{0}=1.7894 \times 10^{-5} \mathrm{~kg} /(\mathrm{m} \cdot \mathrm{s})$ respectively. The heat conductivity coefficient is calculated by:

$$
k(T)=\frac{c_{p}}{P r} \mu(T)=\frac{\gamma c_{v}}{P r} \mu(T)
$$

and the Prandtl number $(P r)$ is 0.72 .

\subsection{Numerical methods and Simulation Code}

The present study has been carried out using a block-structured, finite-volume, high-order ILES code labeled as CNS3D. The code has been previously applied to a broad range of turbulent flow problems, including other canonical problems [84, 88], as well as more complex subsonic, transonic, and supersonic flows [13, 89-94]. CNS3D comprises several discretization (reconstruction) schemes for calculating the variables at the cell faces of the computational cell. In the present study, we have employed the Harten, Lax and van Leer Contact (HLLC) [95] approximate Riemann 
solver, an extension to the original Harten, Lax and van Leer (HLL) solver [96], to define the convective fluxes at the cell face.

The numerical schemes implemented in conjunction with the HLLC solver are:

- MUSCL piece-wise linear $2^{\text {nd }}$-order Monotonized Central (MC) limiter [97, 98];

- MUSCL $3^{\text {rd }}$ (M3) [99] and $5^{\text {th }}$ (M5) order limiters [100, 101];

- WENO $5^{\text {th }}$ (W5) and $9^{\text {th }}$ (W9) order schemes $[102,103]$, which are extensions of the original WENO scheme [104]. WENO schemes and their extensions have been previously investigated in connection with different flow problems both with structured and unstructured grids, see e.g. [90, 105-114] and references therein. Note that the relative smoothness limiter of [115] is employed instead.

The above schemes have been further combined with the LM correction proposed by Thornber et al. [86]; the theoretical development and justification of the LM correction can be found in [87]. It was demonstrated that the LM correction can significantly reduce the numerical dissipation of Godunov-type methods at low Mach numbers via a progressive central differencing of the velocity components in the post-reconstruction phase. An analysis [87] of the source of the turbulent kinetic energy dissipation in upwind schemes revealed that the absolute dissipation of fluctuating kinetic energy is proportional to the temperature multiplied by the change of entropy (assuming an approximately isothermal flow). This neglects the additional dissipation that occurs during isentropic transformation of kinetic energy to internal energy in the form of local compressions and expansions. Using MEA, the evolution of entropy was derived for various compressible numerical schemes and it was demonstrated that the overly dissipative behaviour observed in simulations of homogeneous decaying turbulence is ascribed to numerical dissipation that is proportional to the speed of sound. The LM correction provides a limiting procedure which recovers the accuracy of such schemes with an optimal dissipation in the limit of $M \rightarrow 0$ [86]. In this study, the LM correction is further investigated for low Mach turbulent boundary layers.

\section{Problem set-up}

\subsection{Initial and boundary conditions}

The turbulent channel flow employed in this paper corresponds to the incompressible DNS simulations of Moser et al. [30]. The Reynolds number based on the friction velocity and channel half-height is $R e_{\tau}=395$, which is equivalent to $R e \simeq 6887$ based on the bulk velocity [116]. Previous studies concerning compressible, turbulent channel flows have been conducted at high Mach numbers, however, to examine the effects of the numerical schemes with and without low-Mach correction, the LES studies in this paper are conducted at a Mach number of $M_{\infty}=0.2$.

The size of the non-dimensional domain $\left(L_{x} \times L_{y} \times L_{z}\right)$ is $(2 \pi \times 2 \times \pi)$ in the streamwise $(x)$, wall normal $(y)$ and spanwise (z)-directions, respectively (Figure 1). In the streamwise and spanwise directions, periodic boundary conditions are employed, while in the wall normal direction an adiabatic no-slip wall condition is applied.

In [39], it was shown that many of the scaling relations used to express adiabatic compressible boundary-layer statistics in terms of incompressible boundary layers hold for non-adiabatic cases too. Wall cooling slightly enhances compressibility effects and increases the coherency of turbulent structures, however, its effects remain insignificant even for a supersonic turbulent channel flow. In the compressible DNS channel flow simulations of [37, 38], it was shown that a decreasing wall temperature leads to higher skin friction. In the present study, the adiabatic wall condition was employed in order to examine the accuracy of the numerical methods unhindered by external heat transfer effects, thus obtaining a more meaningful comparison to the incompressible DNS.

The initial conditions of [117] are employed. The streamwise velocity profile is given by a laminar (Poiseuille) parabolic profile with a white noise perturbation $(s=10 \%)$ superimposed. The white noise random signal $(\epsilon)$ was generated using the intrinsic FORTRAN command RAND, which varies according to the index numbering $(i, j, k)$ of each cell along the corresponding spatial direction $[x, y, z]$ using for input the integer function:

$$
N_{i j k}=i+(j-1) \times N_{x}+(k-1) \times N_{y} \times N_{x}
$$




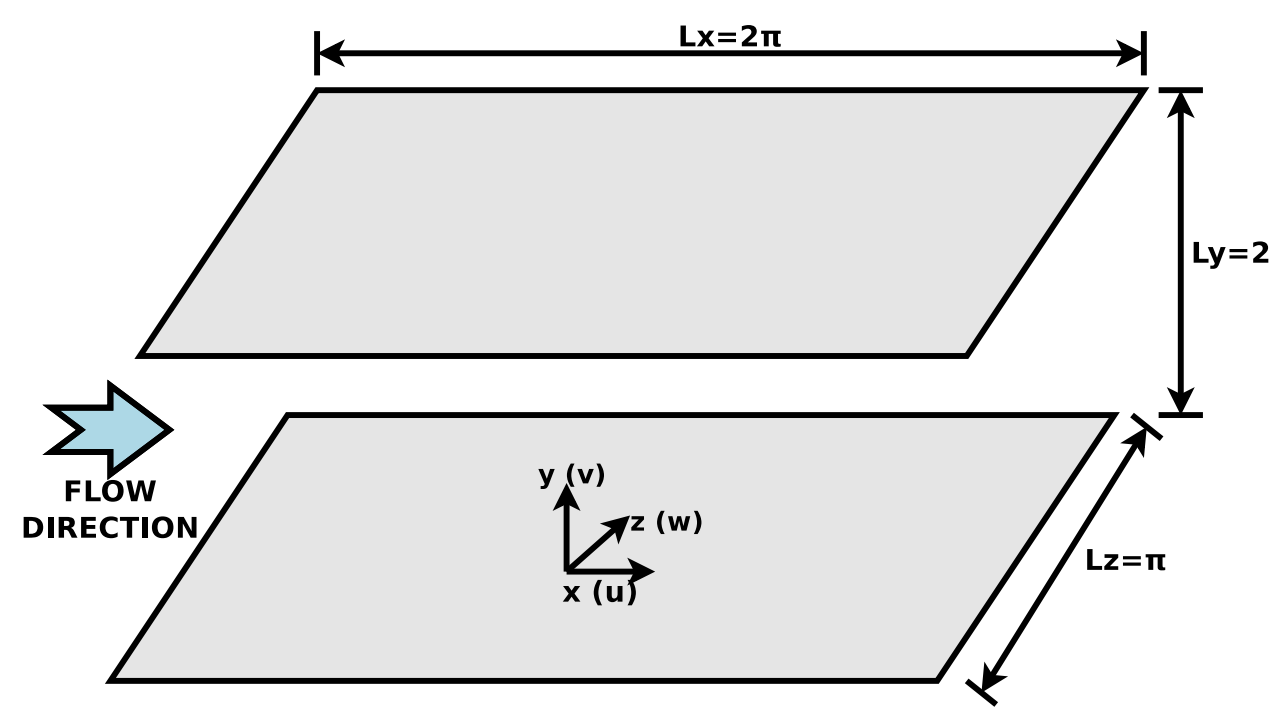

Figure 1: Illustration of channel geometry.

so that $\epsilon=\operatorname{RAND}\left(N_{i j k}\right)$ and $\epsilon \in[-1,1]$. The same velocity perturbation is also used for the spanwise and wall-normal velocity components, while the initial density and pressure are assumed constant throughout the domain. In summary, this leads to the following dimensional initialisation of the primitive variables (at $t=0)$ :

$$
\begin{array}{rlc}
\rho & = & \rho_{\infty} \\
u & = & u_{c}\left[1-\left(\frac{y}{\delta}-1\right)^{2}\right](1+s \epsilon) \\
v= & s \epsilon \times u \\
w= & s \epsilon \times u \\
E & = & \frac{p_{\infty}}{\rho_{\infty}(\gamma-1)}+\frac{1}{2}\left(u^{2}+v^{2}+w^{2}\right)
\end{array}
$$

Isentropic flow relations are used to estimate $p_{\infty}$ based on the free-stream Mach number $M_{\infty}=0.2$. The initial freestream density and pressure values are $\rho_{\infty} \approx 1.20 \mathrm{~kg} / \mathrm{m}^{3}$ and $p_{\infty} \approx 98540 \mathrm{~Pa}$, respectively. The centreline velocity used for obtaining the initial laminar profile is $u_{c}=(3 / 2) u_{\infty}$, where the bulk (free-stream) velocity is $u_{\infty} \approx 67.8 \mathrm{~m} / \mathrm{s}$.

To investigate the grid convergence properties of the schemes, three computational grids were employed containing $64^{3}, 96^{3}$ and $128^{3}$ grid points, respectively; see Table 1 for the details of the grids used in comparison to the DNS. The grid points near the wall were clustered using a two parameter hyperbolic tangent stretching function [118]. Note that the coarse grid $\left(64^{3}\right)$ used in the present ILES is approximately 34 times coarser than the DNS grid, while the medium $\left(96^{3}\right)$ and fine $\left(128^{3}\right)$ grids are 10.4 and 4.4 times coarser than the DNS, respectively. It is also worth mentioning that in the DNS the fine grid resolution is also combined with numerical schemes (spectral methods) that inherently contain little or no numerical dissipation, whereas the present non-oscillatory finite volume methods are inherently dissipative schemes.

\subsection{Forcing term}

Periodic conditions in both the streamwise and spanwise directions are employed for reducing the length of the computational domain required for a fully turbulent flow to develop. To ensure that the mass-flux remains constant throughout the simulation a forcing term is added to the Navier-Stokes equations that acts as an artificial pressure gradient. In [37, 119, 120], a forcing term was developed to augment the momentum and energy equations in order to obtain a constant mass-flux. Though the forcing term was initially developed as an artificial pressure gradient term, 


\begin{tabular}{|c|c|c|}
\hline Grid & $\left(N p_{x} \times N p_{y} \times N p_{z}\right)$ & $y^{+}$ \\
\hline ILES (Coarse) & $65 \times 65 \times 65$ & 1.52515 \\
ILES (Medium) & $97 \times 97 \times 97$ & 1.00016 \\
ILES (Fine) & $129 \times 129 \times 129$ & 0.74404 \\
DNS & $256 \times 193 \times 192$ & 0.03000 \\
\hline
\end{tabular}

Table 1: Number of points and $\mathrm{y}^{+}$value of first grid point from the wall for the present ILES and the DNS of Moser et al. [30].

in its final implementation it was reduced down to a simple body force. Interpretations of this term have been given by different authors $[38,121]$.

For compressible channel flows, forcing terms have been previously proposed by $[119,120]$ for the subsonic regime based on the extension of the incompressible condition derived in [122], as well as by [37] for supersonic channel flows. More recently, [40] proposed a new subgrid term based on the pressure gradient, which is added to the momentum and energy equations. In the compressible LES of [123], the description of the forcing term for compressible channel flow was revisited, so that the streamwise periodic simulation resembles as much as possible that of a spatially evolving fully developed turbulent channel flow. This requires, in addition to an artificial force term in the momentum equation, an artificial heat source term to be added to the internal energy equation.

In the present simulations, a forcing term $f_{t}$ is added to the right hand side (RHS) of the streamwise momentum Equation (2), with the total energy Equation (5) remaining unchanged to conserve the total energy. The forcing term $\left(f_{t}\right)$ is calculated at the beginning of each time step $(N)$ using the mass-flux dissipation from the previous time step. According to $[119,120]$, this yields:

$$
f_{t}^{N}=f_{t}^{N-1}+\frac{\Delta t}{L_{y} L_{z}}\left[\alpha\left(Q^{N+1}-Q_{0}\right)+\beta\left(Q^{N}-Q_{0}\right)\right]
$$

where $\alpha=2 / \Delta t$ and $\beta=0.2 / \Delta t$ are coefficients that calibrate the stability of the predictor step. $Q_{0}, Q^{N}$ and $Q^{N+1}$ are the target mass-flux $Q_{0}=\left(L_{y} L_{z}\right) \rho_{\infty} u_{\infty}$, the mass-flux at the current time-step $(N)$, and the first-order predictor of the mass flux at time-step $N+1$, respectively. $Q^{N+1}$ is given by:

$$
Q^{N+1}=Q^{N}-\Delta t^{N}
$$

where

$$
g^{N}=L_{y} L_{z} f^{N-1}+\left.2 \frac{L_{z}}{R e} \tau_{w}^{N}\right|_{y=0}
$$

In Equation (19) it is assumed that the values of the wall shear stress $\left(\tau_{w}\right)$ for the upper and lower plates are approximately equal at all time instants.

\subsection{Statistical quantities}

For completeness, the key definitions used in this paper are presented below. A space or ensemble average of a variable $\phi$ is denoted by $\bar{\phi}$. Since the streamwise $(x)$ and spanwise $(z)$-directions are homogeneous, the ensemble average is calculated by $\bar{\phi}=\langle\phi\rangle_{x z}$, where $<>_{x z}$ stands for spatial averaging in the $\mathrm{x}-\mathrm{z}$ plane. The Favre average of a variable, denoted by $\widetilde{\phi}$, at a time $t$ is then obtained by:

$$
\widetilde{\phi}=\frac{\overline{\rho \phi}}{\bar{\rho}}=\frac{\langle\rho \phi\rangle_{x z}}{\langle\rho\rangle_{x z}}
$$


The Favre averaged variable can also be further averaged in time as:

$$
\widetilde{\phi}^{t}=\frac{\int \widetilde{\phi}(t) d t}{\int d t}
$$

to obtain a statistically converged mean profile subject to adequate sampling.

The following statistics have been calculated for comparison with the DNS data: (i) streamwise velocity ( $u$ or $\left.u^{+}\right)$; (ii) Reynolds stresses $\left(\operatorname{RS}_{u_{i}^{\prime} u_{j}^{\prime}}\right)$; (iii) skewness $\left(S_{u_{i}^{\prime}}\right)$; and (iv) flatness $\left(F_{u_{i}^{\prime}}\right)$. The three fluctuating components $\left(u^{\prime}, v^{\prime}, w^{\prime}\right)$ are calculated by $u_{i}^{\prime}=u_{i}-\widetilde{u}_{i}^{t}$, where the 'dash' denotes the fluctuating part of the variable.

The Reynolds stresses are normalized by the resolved friction velocity in order to compare against the available DNS data of [30]:

$$
R S\left(u_{i}^{\prime} u_{j}^{\prime}\right)=\left.\frac{<u_{i}^{\prime} u_{j}^{\prime}>_{x z}}{\left(\tilde{u}_{\tau}^{t}\right)^{2}}\right|_{y=0} ^{L_{y}}
$$

where $\left.\right|_{y=0} ^{L_{y}}$ stands for the variable's profile in the $y$-axis.

The skewness and flatness are given, respectively, by

$$
S\left(u_{i}^{\prime}\right)=\left.\frac{<u_{i}^{\prime 3}>_{x z}}{\sqrt[3]{<u_{i}^{\prime 2}>_{x z}}}\right|_{y=0} ^{L_{y}}
$$

and

$$
F\left(u_{i}^{\prime}\right)=\left.\frac{<u_{i}^{\prime 4}>_{x z}}{<u_{i}^{\prime 2}>_{x z}^{2}}\right|_{y=0} ^{L_{y}}
$$

The statistical convergence of skewness and flatness is a good indicator of the fully developed status of the flow, as well as of the adequacy of the data sampling. Note that all statistical profiles obtained at a given instant are further averaged in time using Equation (21).

\section{Results}

To obtain a time window that ensures statistically stationary results, simulations have been carried out over a minimum of ten flow-through times after transition has occurred, using the numerical schemes MC (Monotonized Central, MUSCL $2^{\text {nd }}$-order), M3 (MUSCL $3^{\text {rd }}$-order), M5 (MUSCL $5^{\text {th }}$-order), W5 (WENO $5^{\text {th }}$-order) and W9 (WENO ${ }^{\text {th }}$ order) with and without LM corrections.

The three-dimensional turbulent structures obtained from different simulations are shown by plotting the isosurfaces of $Q$-criterion [124] in Figures 2 to 4 for the schemes without the LM correction and Figures 5 to 7 with the LM correction. $Q$-criterion is an indication of vorticity prevailing over strain and is useful in identifying vortex cores. The $Q$ iso-surfaces show, in a qualitative manner, the ability of the different schemes to resolve turbulent structures. Note that for the calculation of $Q$-criterion the velocity field is non-dimensionalized by the bulk velocity $\left(u_{\infty}\right)$. It is clearly evident that as the order of accuracy of the reconstruction increases, more turbulent structures are resolved. W5 resolves more turbulent structures than M5. The reason is that MUSCL schemes are designed to satisfy positivity-preserving criteria in the framework of the total variation diminishing (TVD) condition [11] that leads to more dissipative schemes.

The LM correction results in a remarkable improvement of the $2^{\text {nd }}, 3^{\text {rd }}$ and $5^{\text {th }}$-order schemes enabling much finer turbulent scales to be resolved, as it is evident when comparing Figures 2-4 with Figures 5-7. The lower the order of accuracy and grid resolution are, the more obvious the effects of the LM correction become. The most important advantage of the LM correction is that it can provide significantly better accuracy on coarser grids than the same numerical scheme might achieve without the correction on a substantially refined grid. The W9 scheme provides the most turbulent-like solutions without using the LM correction. This is because W9 is the least dissipative scheme 
employed, thus the addition of LM corrections does not offer any further improvement and could in fact amplify dispersive errors originating from the truncation error terms (odd order terms).

The results also reveal the mechanism by which the generated vorticity occurring in the viscous layer is 'ejected' due to low speed streaks into the outer boundary layer, thus making it turbulent. This mechanism is responsible for the production of hairpin vortices that get stretched by the ambient shear. These streamwise elongated vortices have also been reported by previous (incompressible) ILES studies [80].

Further analysis of the behaviour of the numerical schemes is now carried out and assessed through quantitative comparisons to the DNS data of [30].

Figure 8 shows the velocity profiles in wall units for different numerical schemes and grid resolutions. The LM correction provides significant improvements for the MC, M3 and M5 schemes and to a lesser degree for the W5 scheme. For example, the second order MC with LM correction on $64^{3}$ resolution, captures the streamwise velocity profile better than the M5 without the LM correction on the $128^{3}$ resolution. On the other hand, the LM correction has hardly any effect on W5, while it slightly deteriorates the profile of W9. In respect of the latter, it should be noted that the 9th -order WENO scheme is the least dissipative but more dispersive than the other methods and this may trigger locally entropy-violating solutions. The LM correction tends to reduce even further the numerical dissipation of the scheme, thus having adverse effects on the numerical error. For all grid resolutions employed, the W9 profile consistently gave results closer to DNS than any other scheme employed here. On the $128^{3}$ grid, the W9 solution is practically identical to the DNS data.

The results for the Reynolds stresses (RS) are shown in Figures 9-12 revealing that the most accurate solution is obtained by W9. The LM correction significantly improves the accuracy of all schemes apart from W9. It appears that the W9 scheme encompasses sufficient dissipation and the LM correction has a significant anti-dissipative effect that adversely affects the accuracy. The lower the order of accuracy of the scheme is, the greater the effect of the LM correction becomes. The W5 scheme gives better results than M5 for the $\operatorname{RS}\left(u^{\prime} u^{\prime}\right)$ and $\operatorname{RS}\left(u^{\prime} v^{\prime}\right)$ and similar results to M5 (with LM correction) for RS $\left(w^{\prime} w^{\prime}\right)$. Overall, the W5 and W9 performed better than any of the MUSCL schemes. The WENO schemes, particularly W9, give extremely accurate results that closely match the DNS profiles. The most noticeable result here is the significant over-prediction of the $\operatorname{RS}\left(u^{\prime} u^{\prime}\right)$ and under-prediction of $\operatorname{RS}\left(u^{\prime} v^{\prime}\right)$ by all schemes apart of W9. The W9 profile of $\mathrm{RS}\left(w^{\prime} w^{\prime}\right)$ on the $64^{3}$ grid appears to be odly shaped after the maxima point but increasing the grid resolution improves the shape of the profile. Additionally, the W9 was the only scheme capable of resolving the initial rise of $\operatorname{RS}\left(w^{\prime} w^{\prime}\right)$ up to $y=0.1$, while it begins to deviate from the DNS at about $y=0.15$ (similarly to other schemes).

Regarding the location of the maximum Reynolds stress, the streamwise and wall-normal components are the most challenging to resolve. As aforementioned, the W9 is the only scheme that gives satisfactory results. Decreasing the order of accuracy leads to a gradual shift of the peak location towards the midstream. The wall-normal velocity Reynolds stress $\operatorname{RS}\left(w^{\prime} w^{\prime}\right)$ is the least accurately captured due to the unresolved turbulent scales associated with the small near-wall fluctuations. These are masked by the increasing numerical dissipation produced as the order of accuracy of the numerical scheme is decreased and as the local Mach number reaches the zero limit near the wall. With regards to $\operatorname{RS}\left(u^{\prime} v^{\prime}\right)$, all schemes show an overall good agreement. All Reynolds stress terms gradually converge to the DNS peak values in the proximity of $y=0.1\left(y^{+} \approx 40\right)$, an indication of the prevalence of turbulent production located near the end of the buffer layer.

High-order turbulent statistics provide a stringent accuracy test for numerical schemes because they can reveal the extent of under-resolution and numerical errors. Flatness (kurtosis) is used here to identify locations where the maxima of the distribution of the velocity component fluctuations occur. High values of flatness indicate regions of the flow where the magnitude of the fluctuations can be relatively high to its mean, while non-zero values of skewness reveal if the velocity fluctuation has a direction of preference. The flatness and skewness of the velocity components are presented in Figures 13 to 18. Typically, the flatness is higher near the wall for all velocity components as shown in Figures 13, 15 and 17, a manifestation of the intermittent nature of the flow in the viscous sub-layer. The results show a gradual convergence to DNS as the order of accuracy or grid resolution is increased. However, it is clear that the order of accuracy of the numerical scheme has a much greater impact on the resulting profiles than the grid resolution. The implementation of the MUSCL schemes in conjunction with the LM correction results in DNS-like distributions on the $96^{3}$ grid something that is unattainable even on the $128^{3}$ grid without the LM correction.

At grid resolution $128^{3}$ the results for the streamwise flatness and skewness profiles (Figures 13, 14, 16 and 18) show improvement only for the W9 scheme. The schemes cannot capture the DNS spanwise skewness profile 


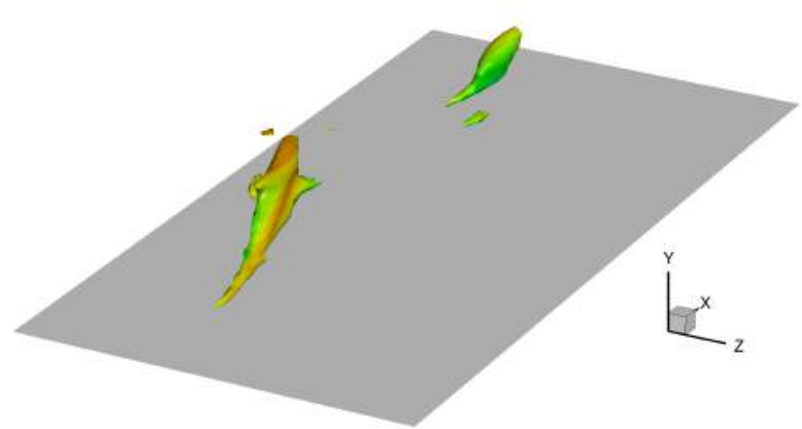

(a) $M C 2^{\text {nd }}$

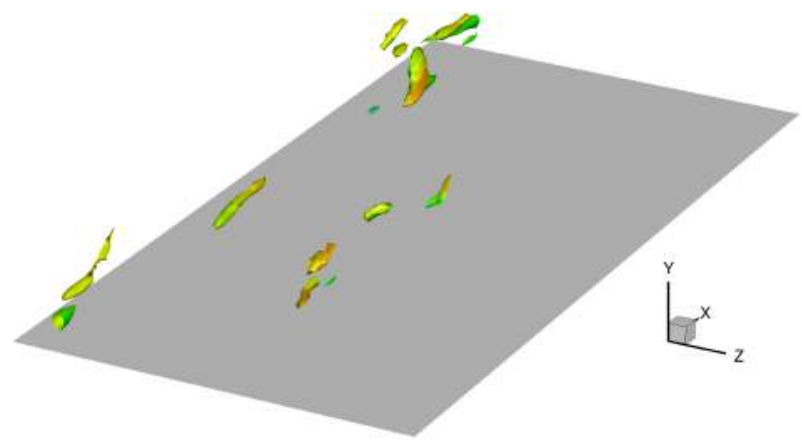

(c) MUSCL $5^{\text {th }}$

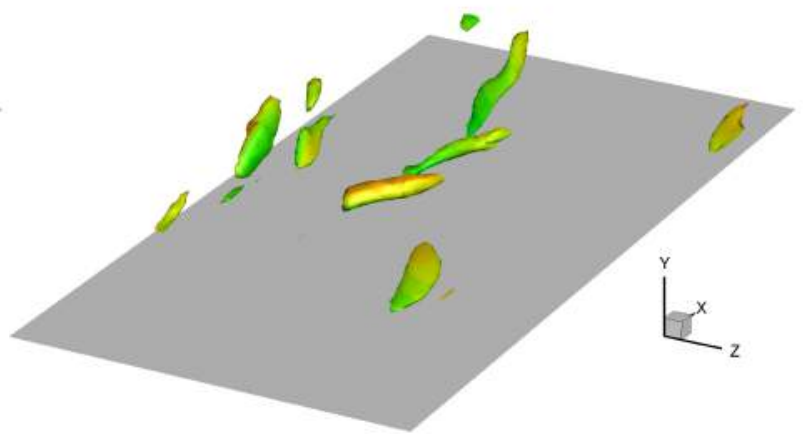

(b) MUSCL $3^{\text {rd }}$

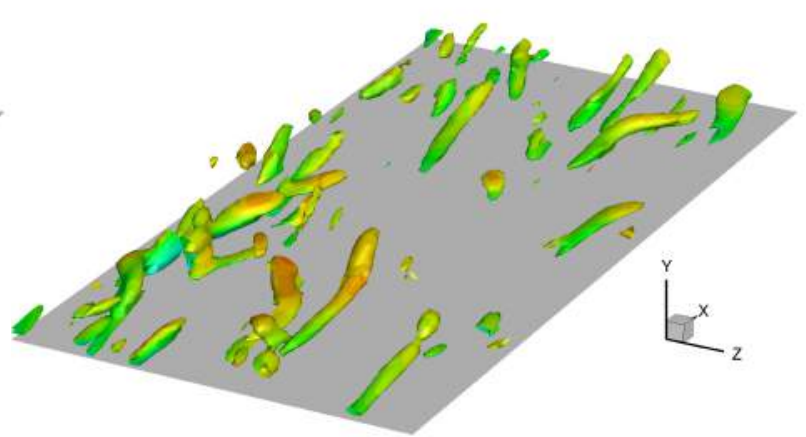

(d) WENO $5^{\text {th }}$

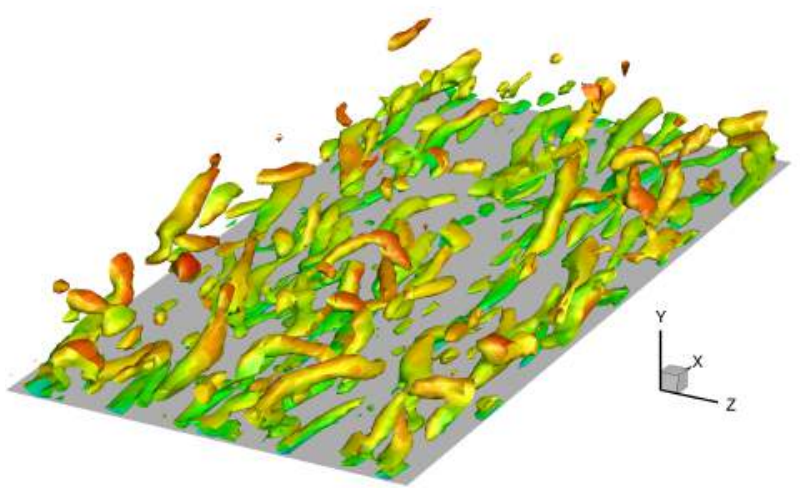

(e) WENO $9^{\text {th }}$

Figure 2: $Q$-criterion iso-surfaces on $64^{3}$ grid (iso-value $=0.5$ colored by streamwise velocity.) 


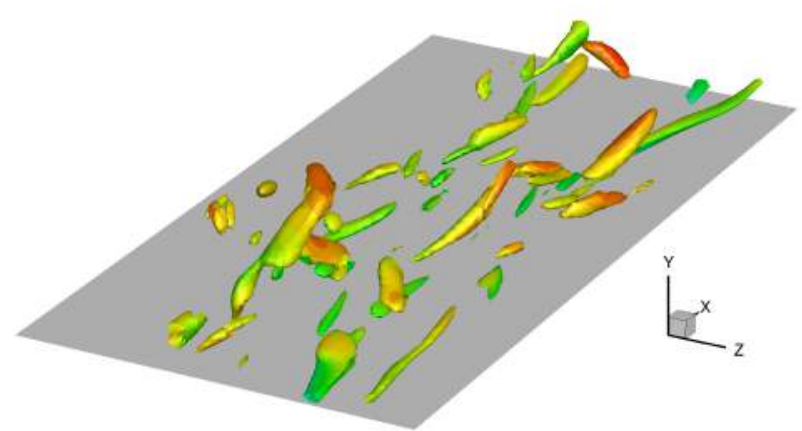

(a) MC $2^{\text {nd }}$

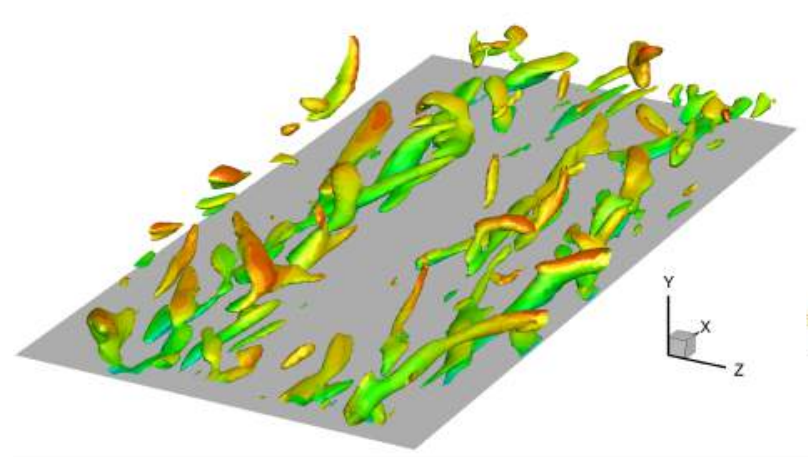

(c) MUSCL $5^{\text {th }}$

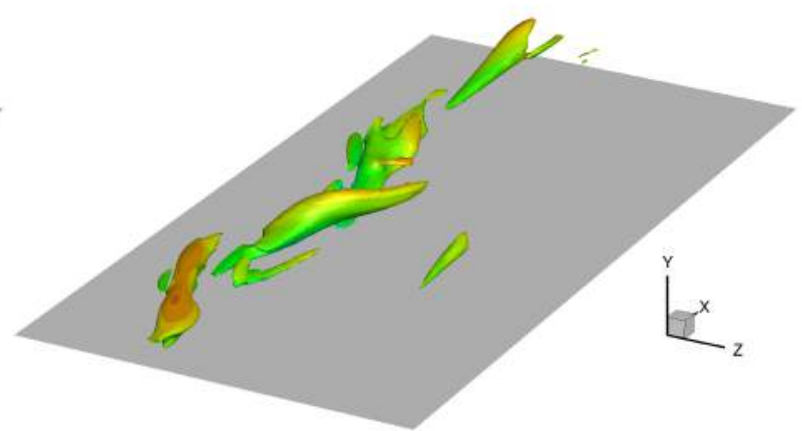

(b) MUSCL $3^{\text {rd }}$

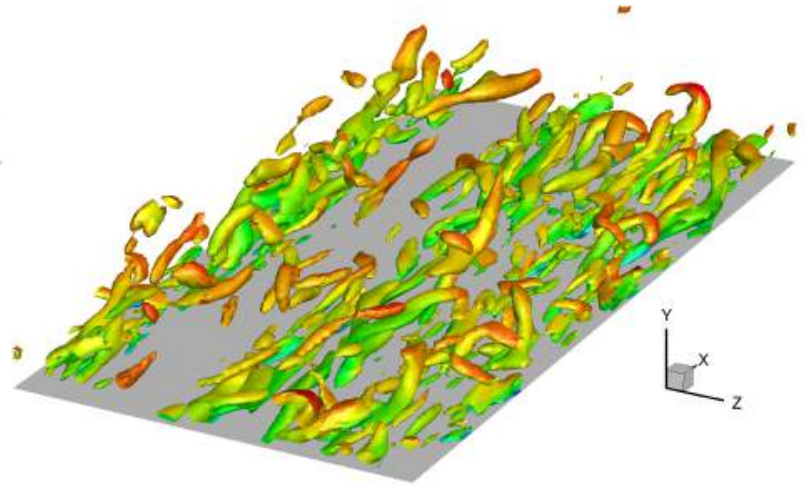

(d) WENO $5^{\text {th }}$

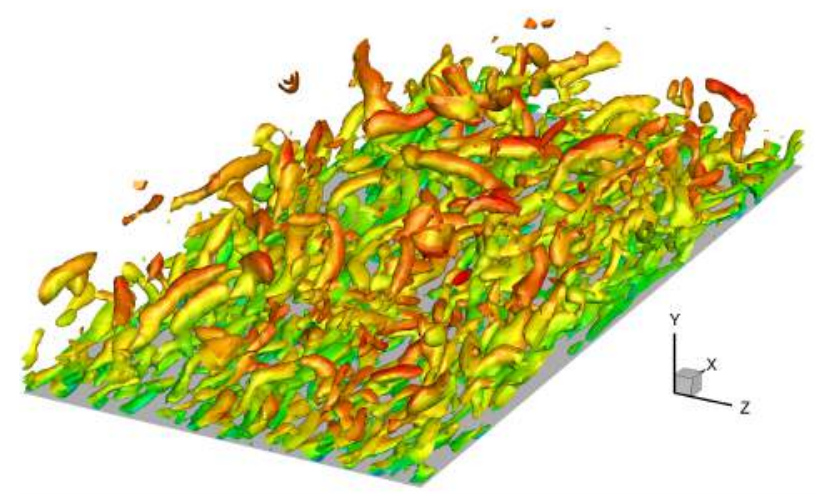

(e) WENO $9^{\text {th }}$

Figure 3: $Q$-criterion iso-surfaces on $96^{3}$ grid (iso-value $=0.5$ colored by streamwise velocity.) 


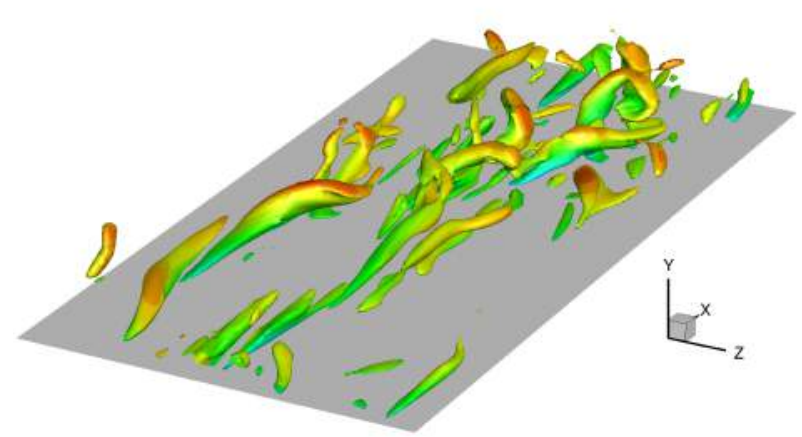

(a) MC $2^{\text {nd }}$

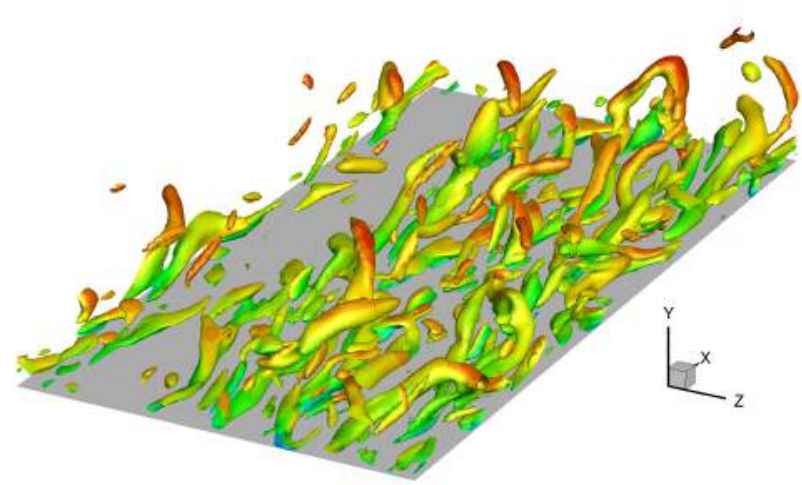

(c) MUSCL $5^{\text {th }}$

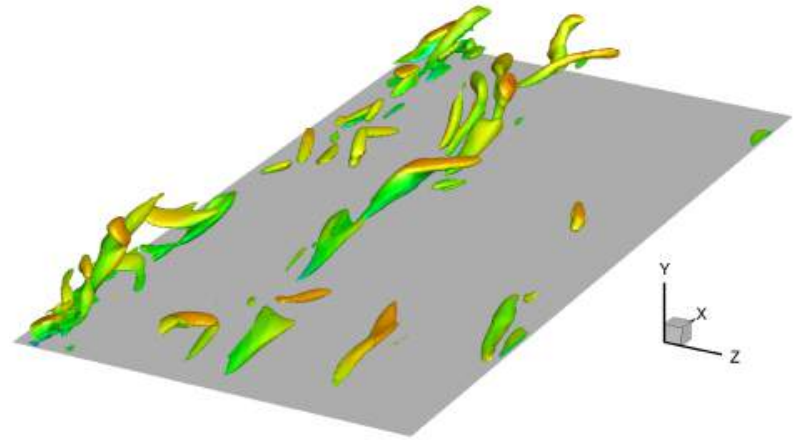

(b) MUSCL $3^{\text {rd }}$

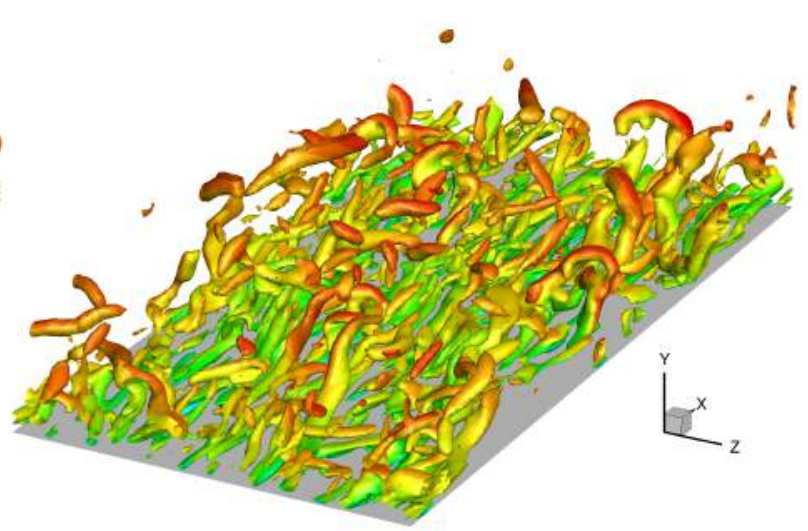

(d) WENO $5^{\text {th }}$

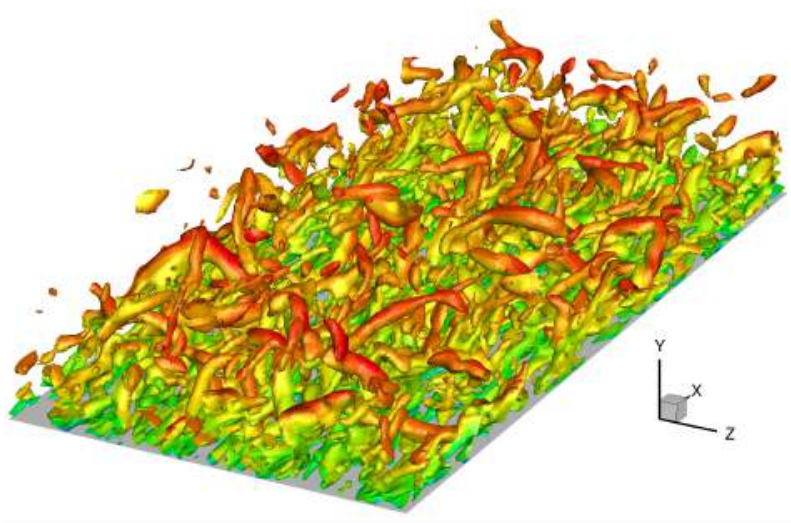

(e) WENO $9^{\text {th }}$

Figure 4: $Q$-criterion iso-surfaces on $128^{3}$ grid (iso-value $=0.5$ colored by streamwise velocity.) 


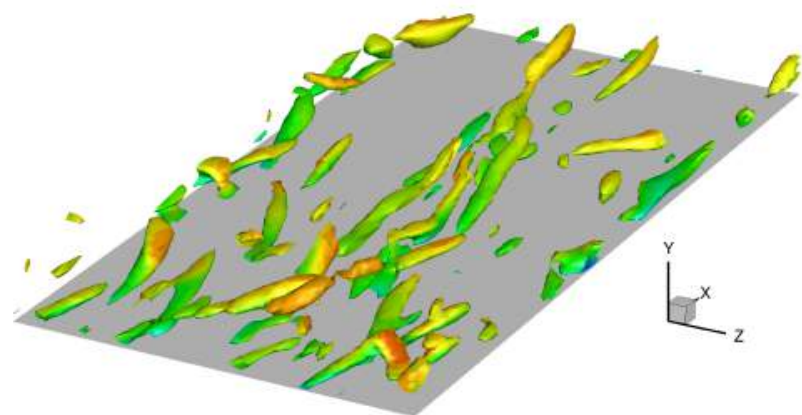

(a) $\mathrm{MC} 2^{\text {nd }}$

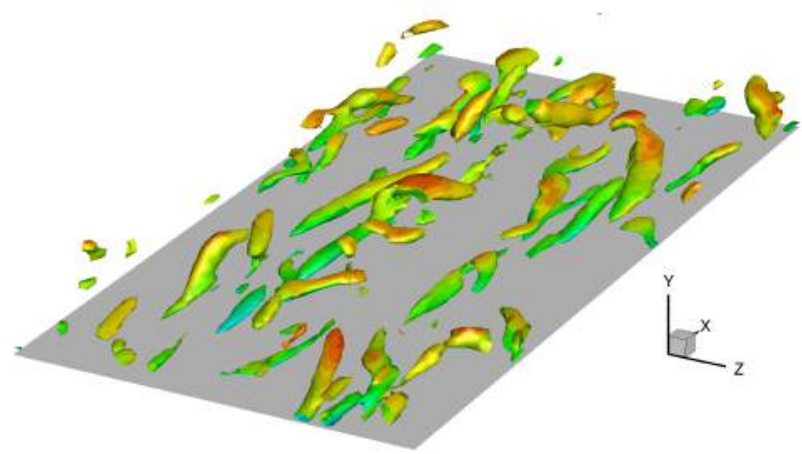

(c) MUSCL $5^{\text {th }}$

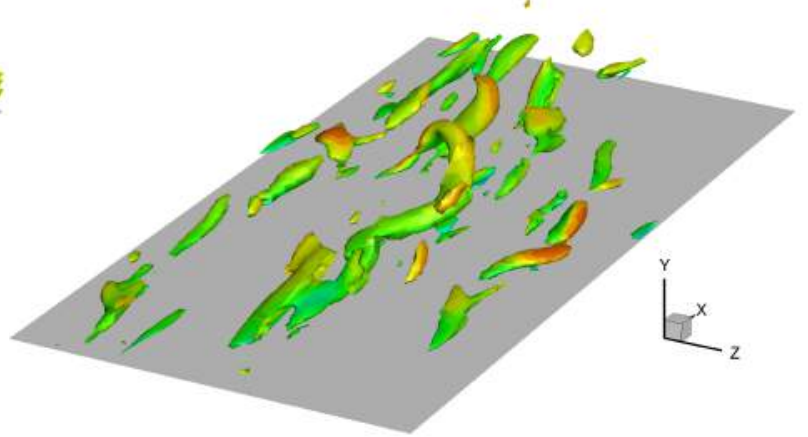

(b) MUSCL $3^{\text {rd }}$

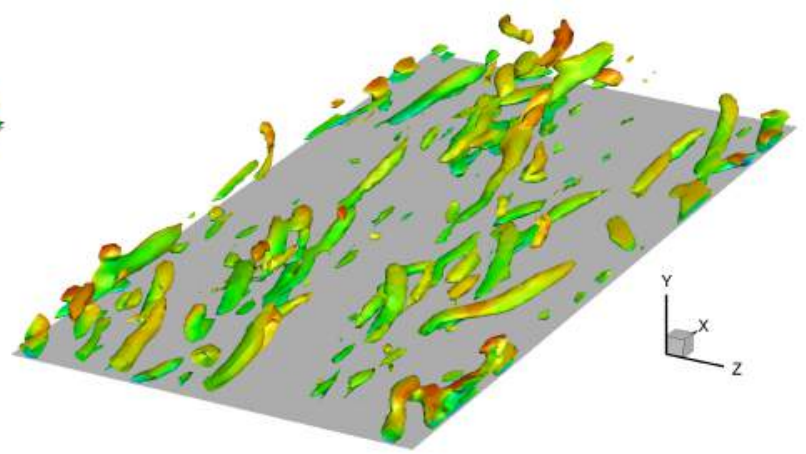

(d) WENO $5^{\text {th }}$

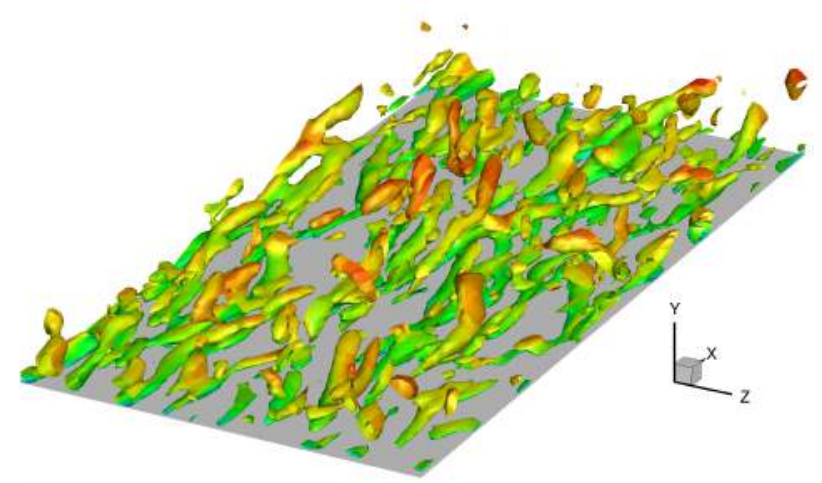

(e) WENO $9^{\text {th }}$

Figure 5: $Q$-criterion iso-surfaces on $64^{3}$ grid with LM corrections (iso-value $=0.5$ colored by streamwise velocity.) 


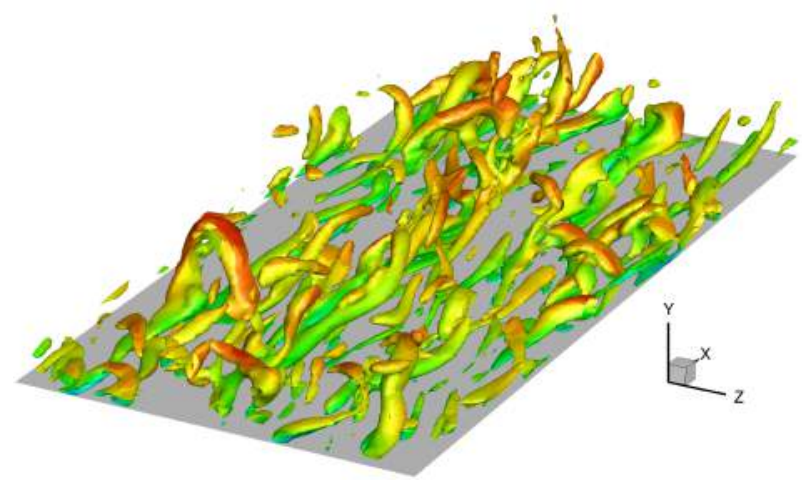

(a) MC $2^{\text {nd }}$

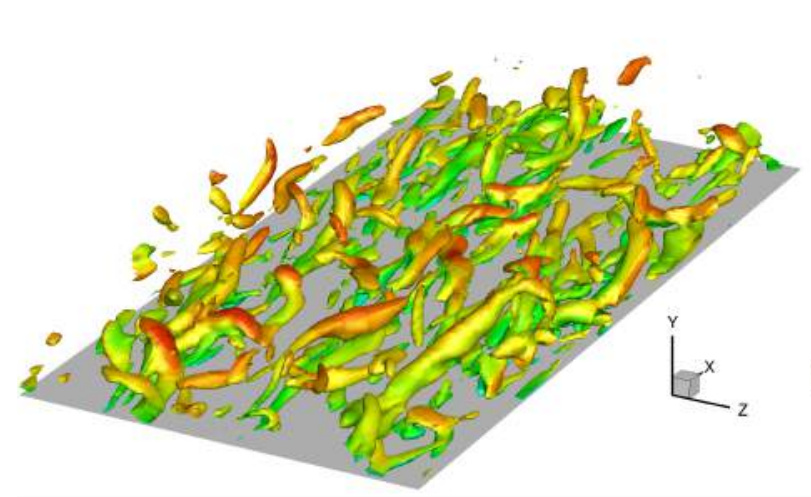

(c) MUSCL $5^{\text {th }}$

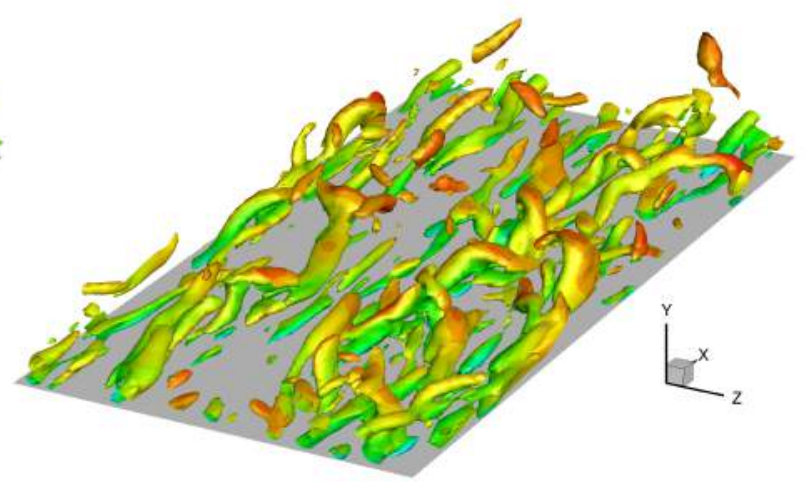

(b) MUSCL $3^{\text {rd }}$

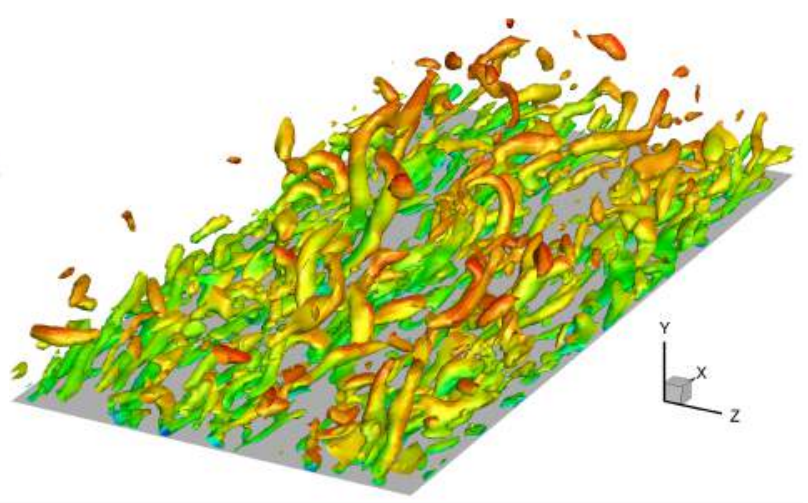

(d) WENO $5^{\text {th }}$

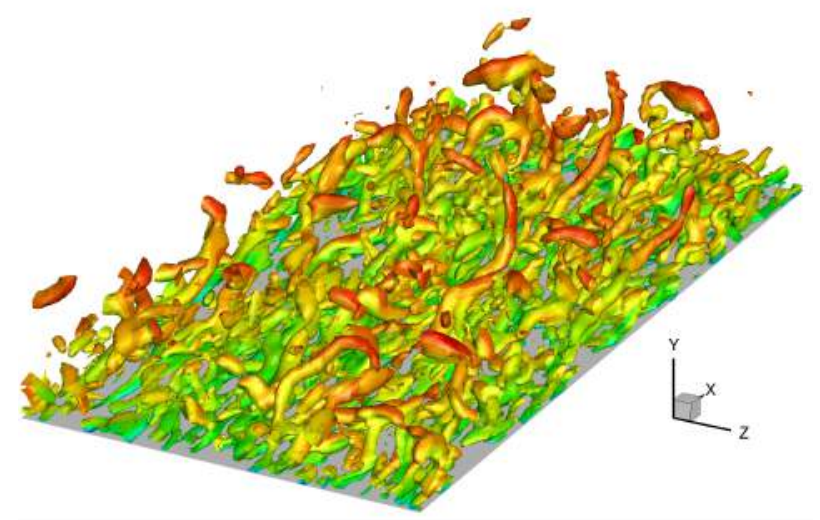

(e) WENO $9^{\text {th }}$

Figure 6: $Q$-criterion iso-surfaces on $96^{3}$ grid with LM corrections (iso-value $=0.5$ colored by streamwise velocity.) 


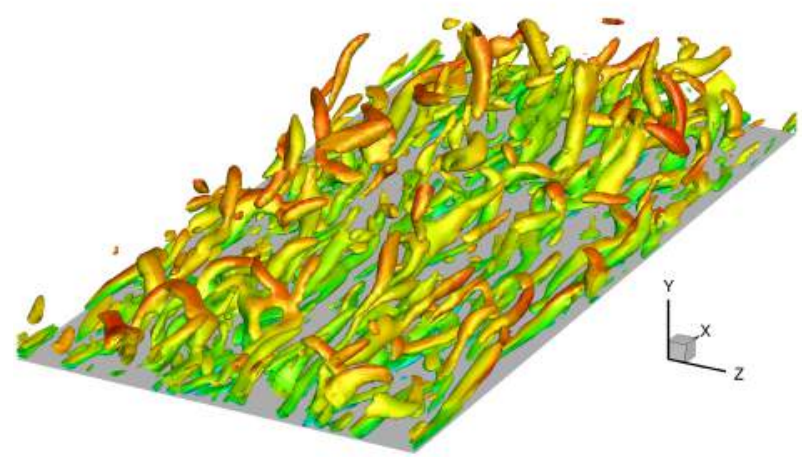

(a) MC $2^{\text {nd }}$

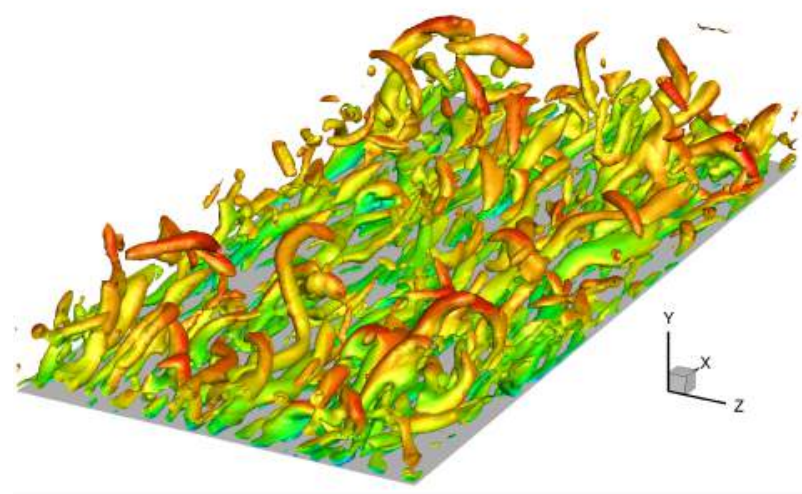

(c) MUSCL $5^{\text {th }}$

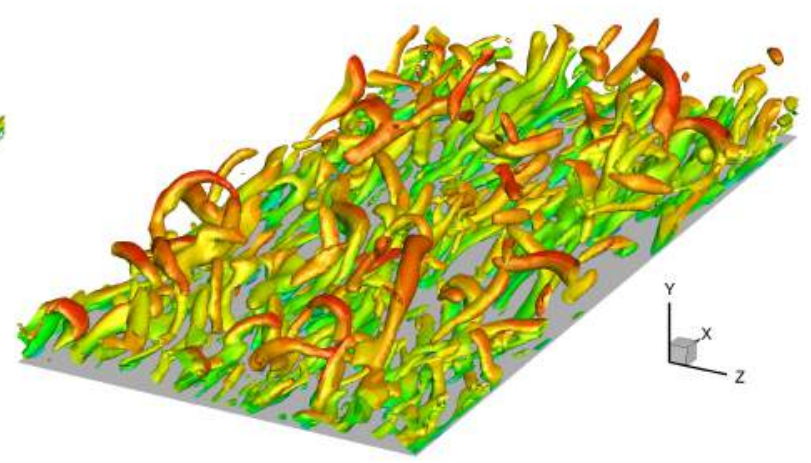

(b) MUSCL $3^{\text {rd }}$

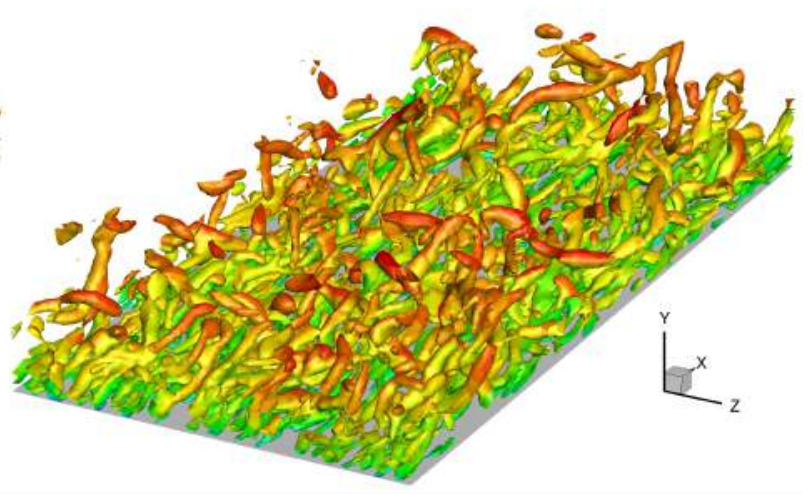

(d) WENO $5^{\text {th }}$

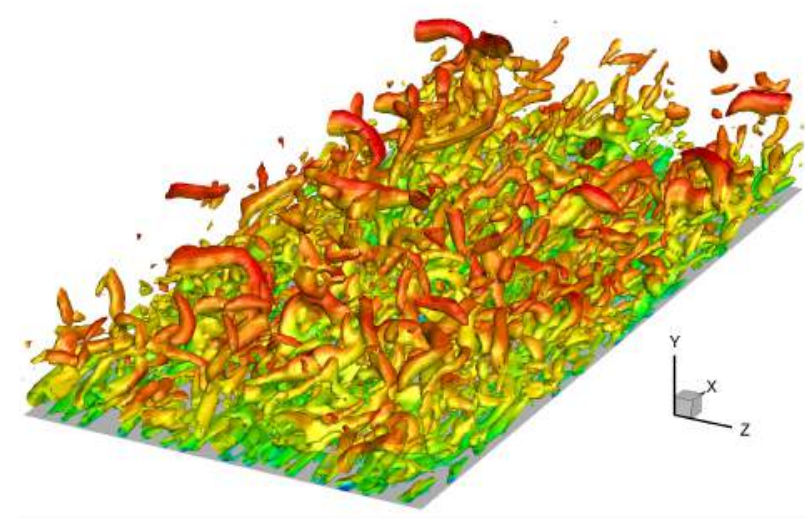

(e) WENO $9^{\text {th }}$

Figure 7: $Q$-criterion iso-surfaces on $128^{3}$ grid with $L M$ corrections (iso-value $=0.5$ colored by streamwise velocity.) 


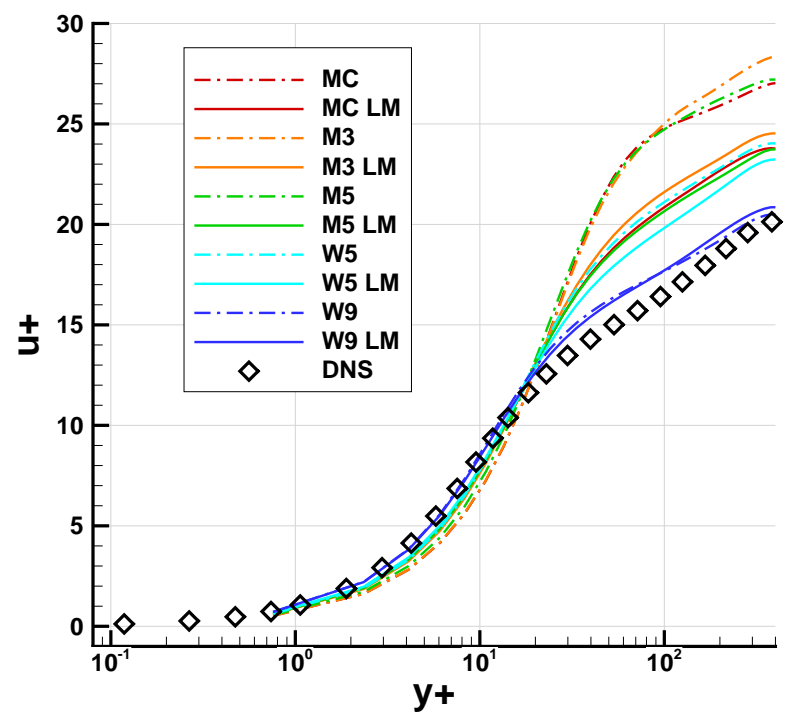

(a) $64^{3}$ grid

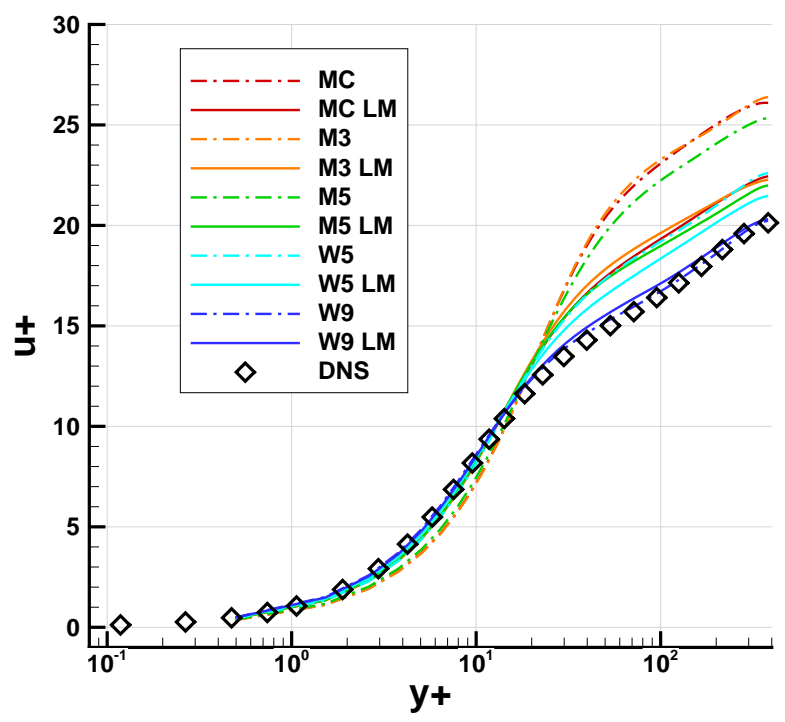

(b) $96^{3}$ grid

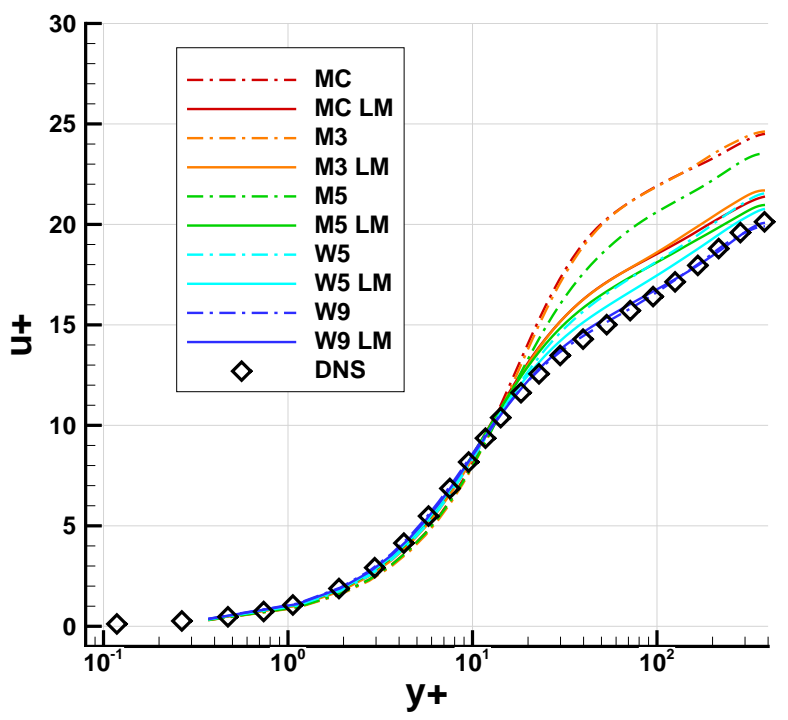

(c) $128^{3}$ grid

Figure 8: Velocity profiles in wall units for different schemes and grid resolutions 


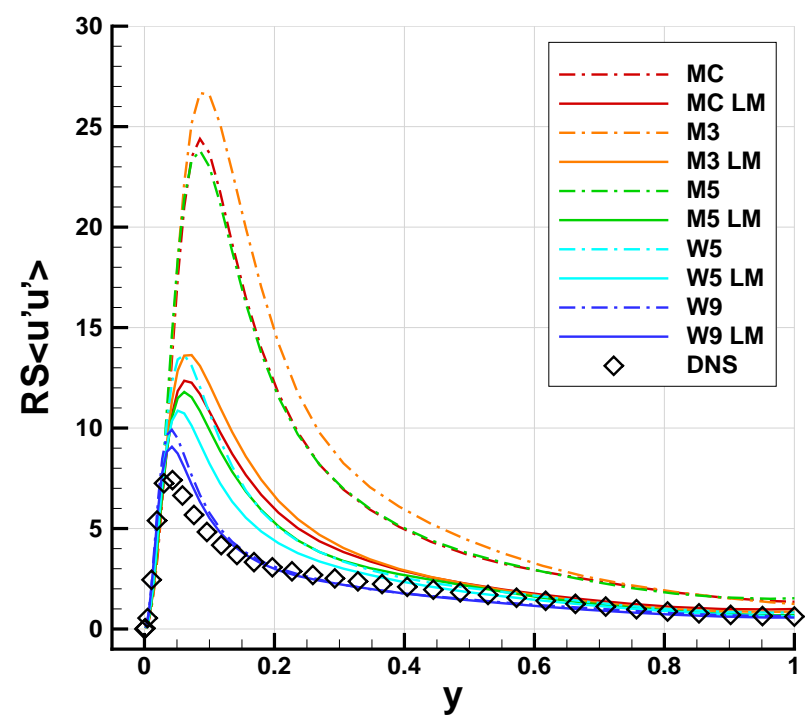

(a) $64^{3}$

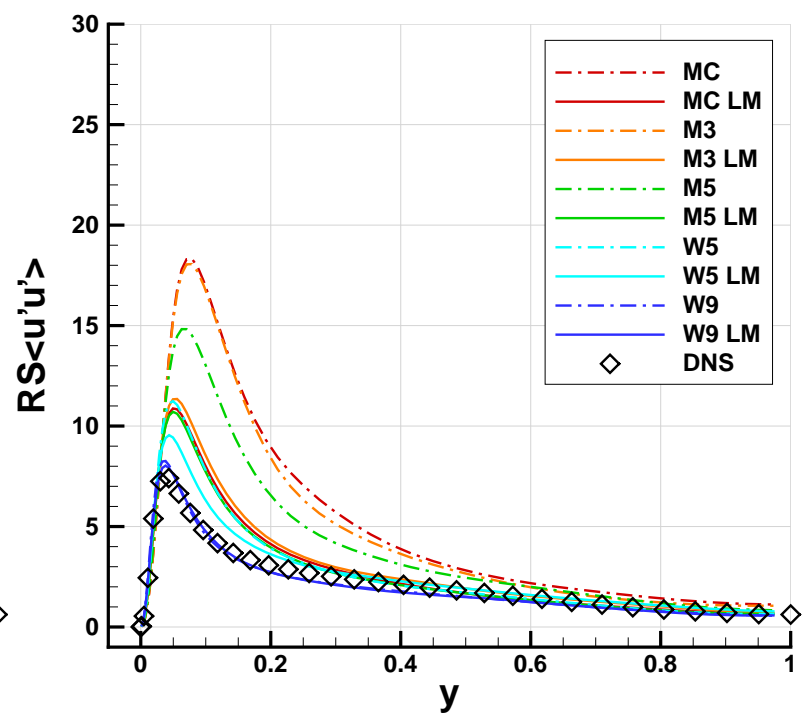

(b) $96^{3}$

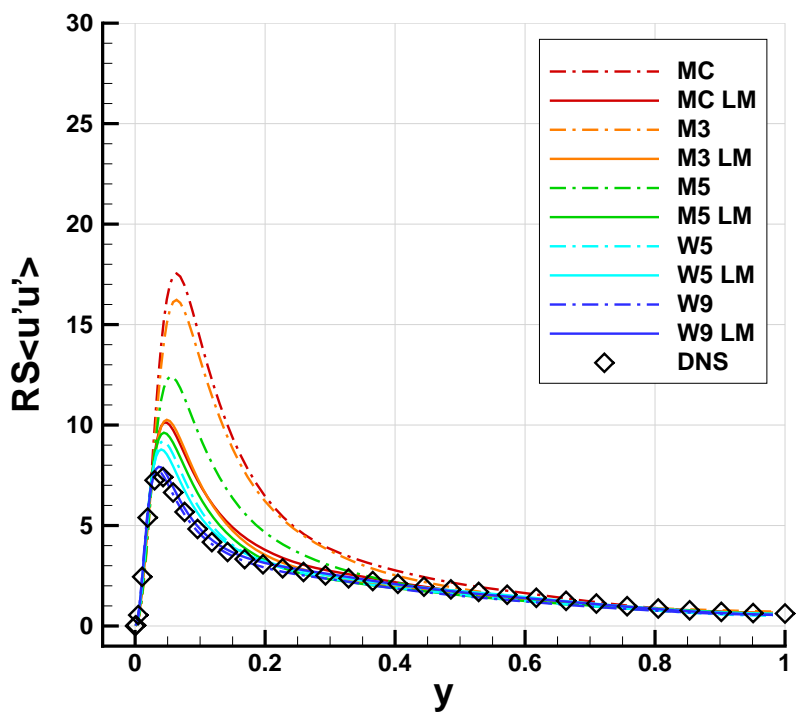

(c) $128^{3}$

Figure 9: The streamwise velocity $\operatorname{RS}\left(u^{\prime} u^{\prime}\right)$ calculated by different ILES schemes 


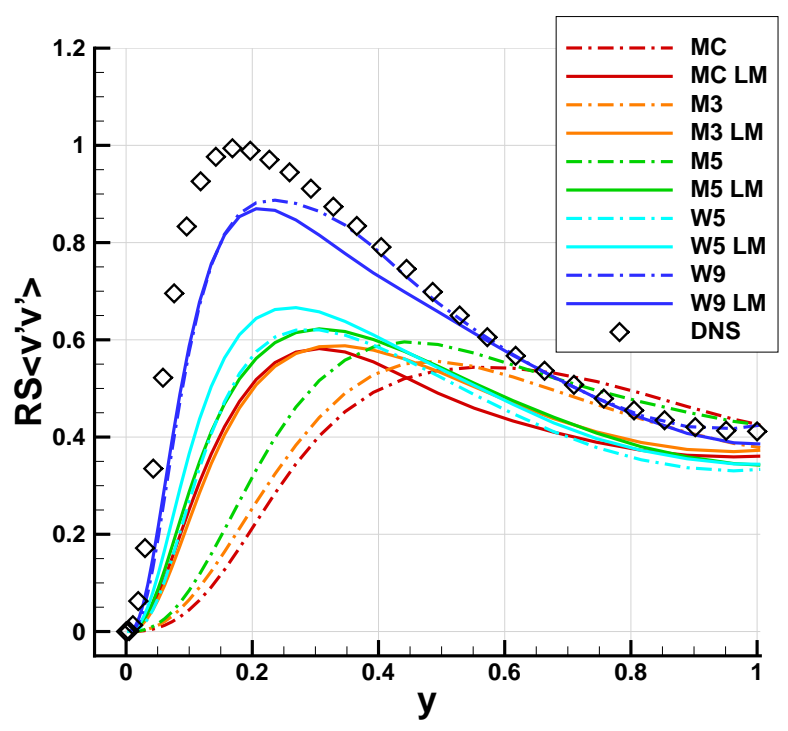

(a) $64^{3}$

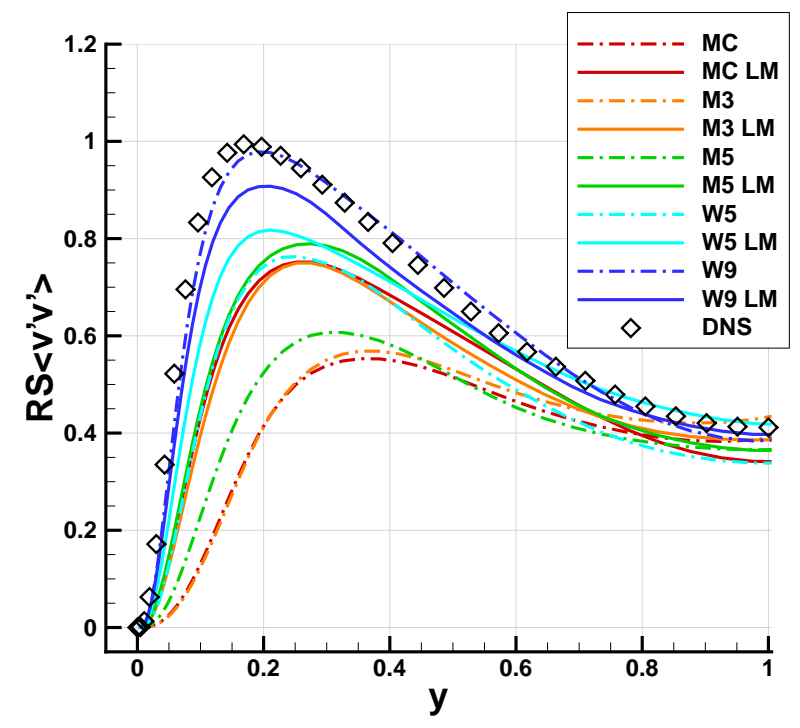

(b) $96^{3}$

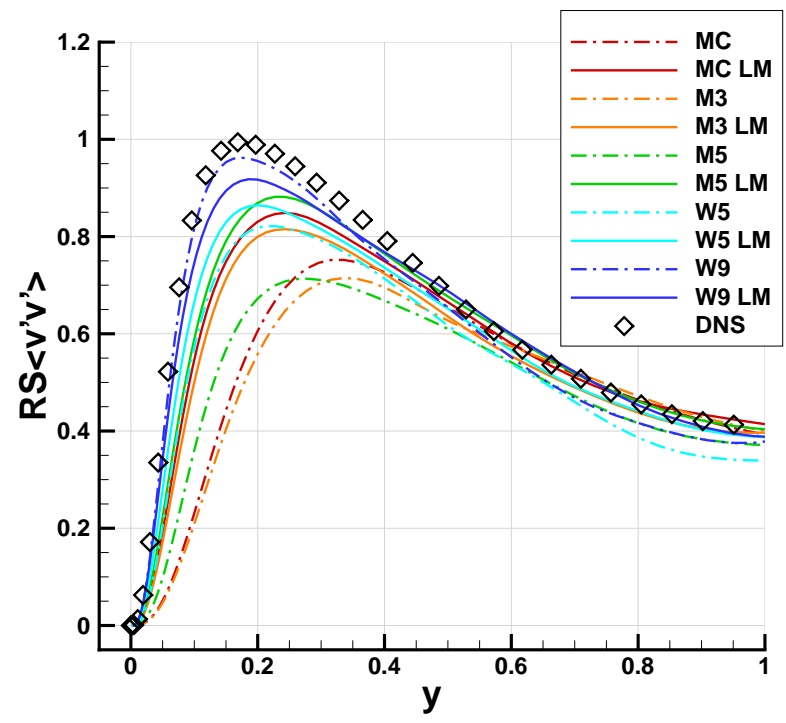

(c) $128^{3}$

Figure 10: The wall-normal velocity $\operatorname{RS}\left(v^{\prime} v^{\prime}\right)$ calculated by different ILES schemes 


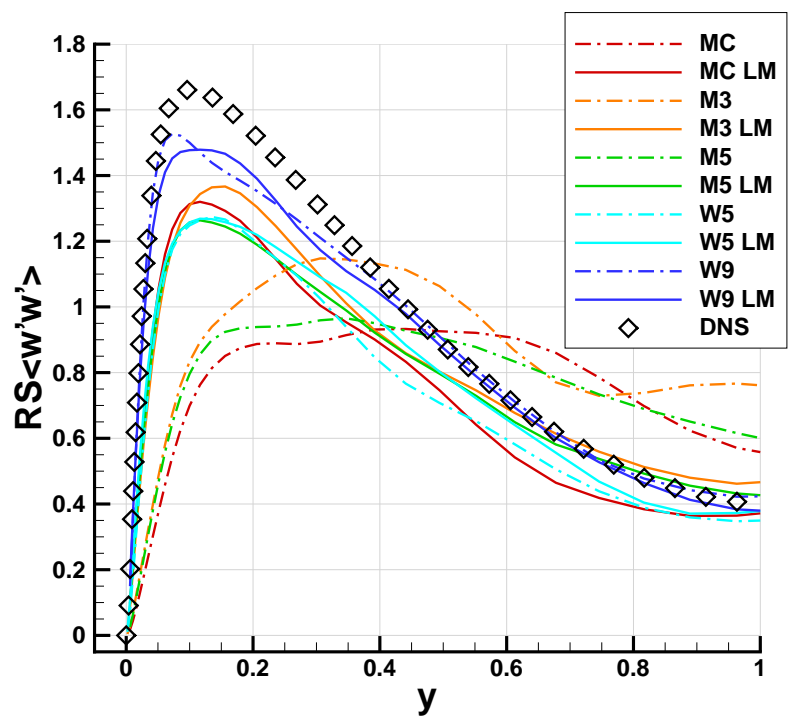

(a) $64^{3}$

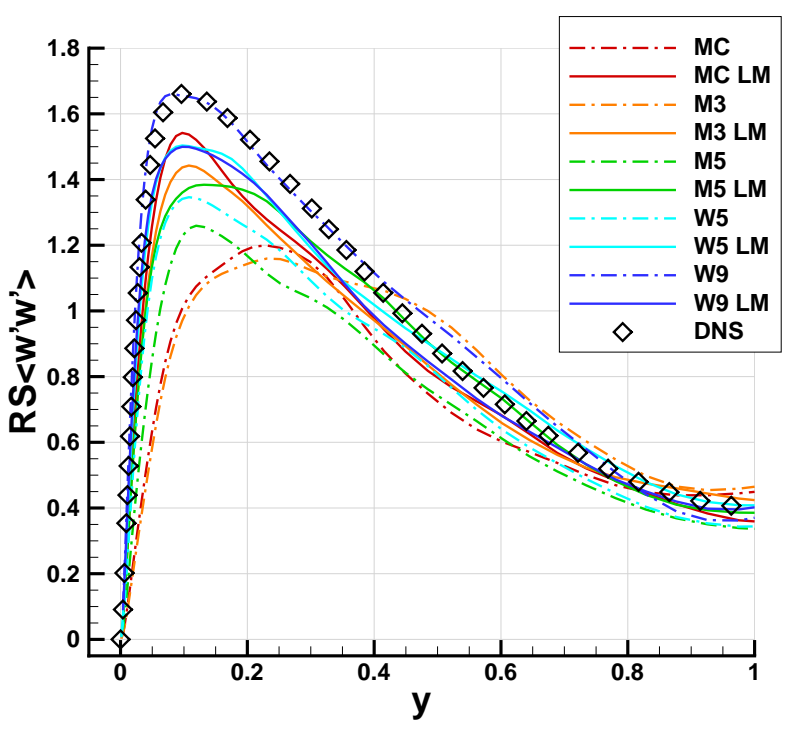

(b) $96^{3}$

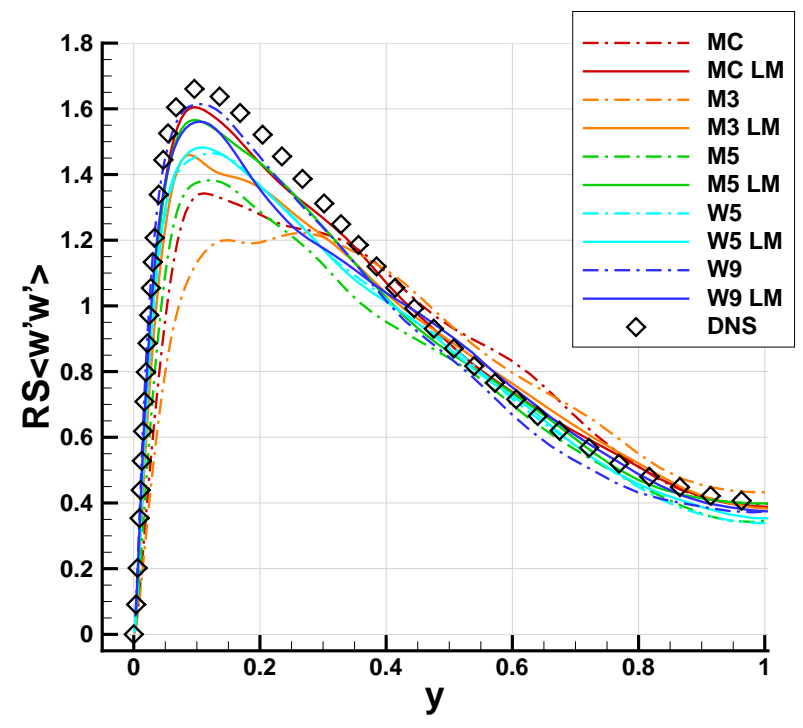

(c) $128^{3}$

Figure 11: The spanwise velocity $\operatorname{RS}\left(w^{\prime} w^{\prime}\right)$ calculated by different ILES schemes 


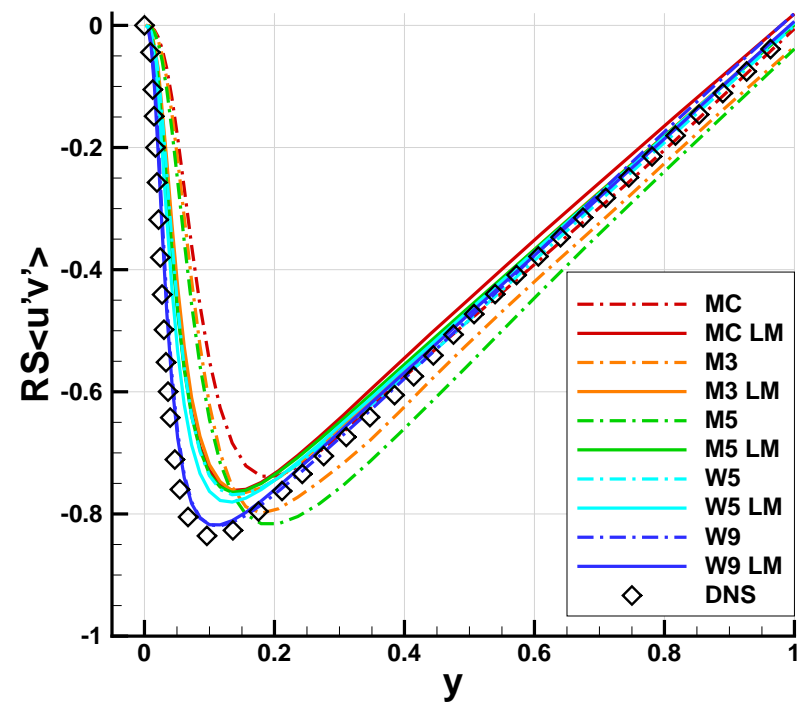

(a) $64^{3}$

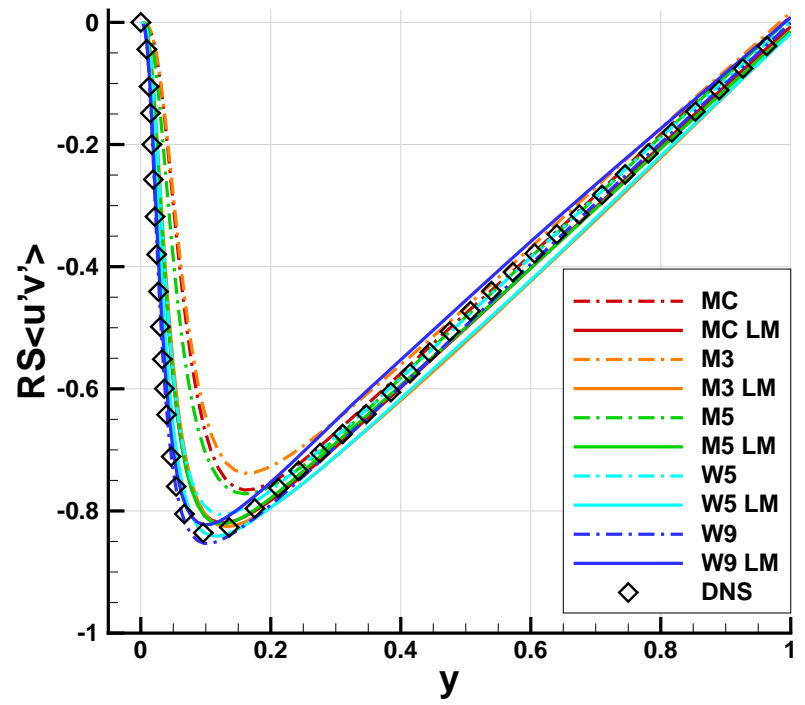

(b) $96^{3}$

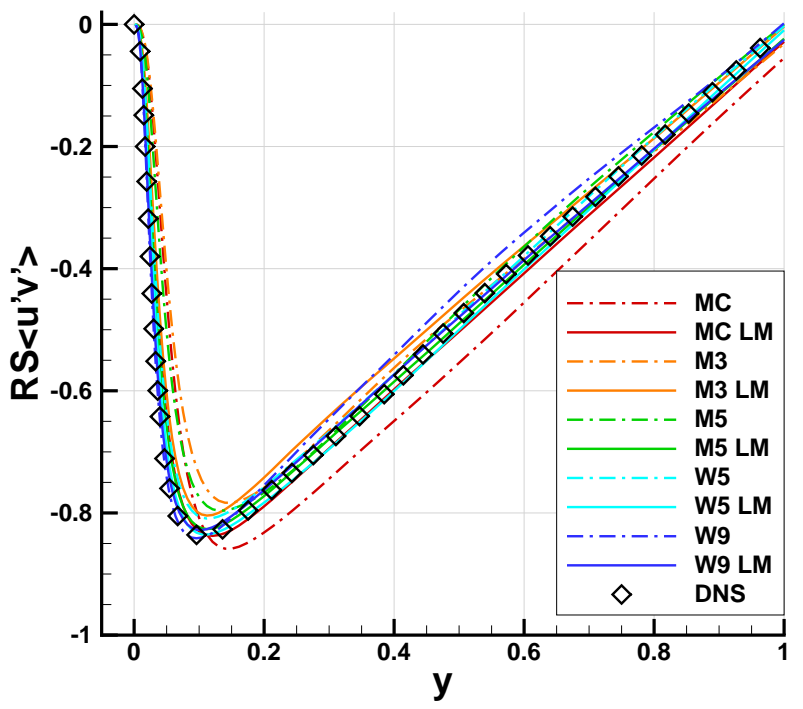

(c) $128^{3}$

Figure 12: The streamwise and wall-normal velocities $\operatorname{RS}\left(u^{\prime} v^{\prime}\right)$ calculated by different ILES schemes 
$\mathrm{S}\left(w^{\prime}\right)$ near the midstream, which takes very small values fluctuating about zero. Increasing the grid resolution helps in obtaining a near wall flatness value that gradually approaches the DNS, and holds true for all the velocity components. This is most prominently visible for the W9 scheme. Regarding skewness, refining the grid further causes the nearwall peak value for the streamwise and wall-normal components to converge closer to the DNS value. Again, this appears to be most evident for the W9 scheme.

The wall-normal skewness profiles of some schemes, in particular the MUSCL scheme, exhibit an unusual positive peak value near the wall prior to converging towards the DNS negative value peak. This is due to the limiting process of the MUSCL and WENO schemes in the near wall region. It is known that strongly imposed no-slip conditions often lead to inaccurate resolution of the near wall turbulence, particularly for coarse boundary-layer meshes. To circumvent this shortcoming, a weakly imposed Dirichlet boundary conditions was suggested [125] and later applied to a turbulent channel flow [126], however, showing that such an approach is less effective for grids employing a stretching function. This kind of condition was not investigated during the course of the present study.

Similar to the observations made for the mean velocity and Reynolds stress profiles, the higher order statistics also confirm that: (i) the $9^{\text {th }}$-order WENO scheme without LM corrections gives overall the best results; (ii) the LM correction has a more pronounced effect on the lower order schemes; and (iii) that the WENO schemes capture the turbulent statistics more accurately than the MUSCL schemes. The W9 is the only scheme that predicts the streamwise flatness profile adequately on the under-resolved $64^{3}$ grid, satisfactorily on the $96^{3}$ and gives a near-DNS solution on the $128^{3}$ grid. The rest of the schemes exhibit noticeable discrepancies to the DNS data, though in some cases, e.g. the wall-normal skewness (Figure 18), the M5 and W5 schemes also perform reasonably well.

Figures 20 and 21 show the difference between the schemes employed and DNS for the streamwise Reynolds stress, flatness and skewness. The numerical schemes in conjunction with the LM correction provide fairly accurate results for the streamwise Reynolds stress $y>0.7$. The least accurate schemes are the M2 and M3 schemes, especially for $y<0.7$, with significant discrepancies near $y=0.1\left(y^{+} \approx 40\right)$, which corresponds to the log-law region. The differences between the schemes and DNS are reflected more clearly on the flatness and skewness. The differences are reduced as the grid is refined. Moreover, the LM correction clearly improves the accuracy of all schemes apart from the W9.

The grid-convergence characteristics of the best schemes, namely the M5 and W9 schemes, are separately shown in Figure 22 and Figure 23, respectively. The grid convergence behavior of the M5 suggests that it could benefit from finer resolution in the near-wall region. Note that since the peak location of the streamwise velocity Reynolds stress is predicted fairly accurately, it is the magnitude which is overestimated. An underlining cause for this could be the under-prediction of the wall-normal and, more importantly, spanwise velocity Reynolds stresses, as previously shown in Figures 10 and 11. Hence, it is argued that the M5 scheme would benefit mostly by increasing the spanwise resolution. The W9 scheme gives the most accurate results, very close to DNS even for the $96^{3}$ resolution.

In Figures 24 to 27, a comparison of the MUSCL and WENO schemes is further carried out for the energy spectra of the spatial fluctuation of the velocity components in the streamwise and spanwise (homogeneous) directions. The best, highest-order variants from these schemes have been selected, namely the M5 and W9, on the $128^{3}$ grid at two locations, one in the mid-stream at $y^{+}=395$, and the other near the wall at $y^{+} \approx 30$ within the buffer layer. It is shown that the W9 scheme provides energy spectra that closely match in magnitude and shape those of the DNS.

The LM correction improves all the energy spectra obtained by the MUSCL schemes. Regarding the M5 scheme, the improvement is apparent by the substantial increase of the energy spectrum, particularly in the low wavenumber regime at $y^{+}=395$ but also at $y^{+} \approx 30$. Despite the above improvements, the magnitude of the energy spectra still remains lower than those obtained by the DNS. The susceptibility of the W9 scheme to the LM correction is mostly evident near the grid cut-off wavenumbers and is caused by the interaction between the dispersive truncation errors and the LM correction. Furthermore, due to the low numerical dissipation in the W9 scheme, the amplified dispersive errors are not sufficiently damped, thus manifesting in the numerical solution.

In the case of $y^{+}=395$, there is an unnatural leveling-off of the energy spectra near the grid-cell (implicit filter) cut-off wavenumber due to the lower mesh resolution in this area. This behaviour is reminiscent of that witnessed in under-resolved simulations using spectral methods and agrees with previous findings in the literature [19, 51-54, 59], where it has also been suggested [53, 55, 56] that explicit LES filtering should be introduced at widths greater than that of the cell size in order to reduce, or avoid altogether, the complex interactions between the numerical (implicit) and SGS model (explicit) dissipation. In contrast to the midstream location, the spectra near the wall do not exhibit such (strong) adverse effects from aliasing and truncation errors. The observations made about the effects of the LM 


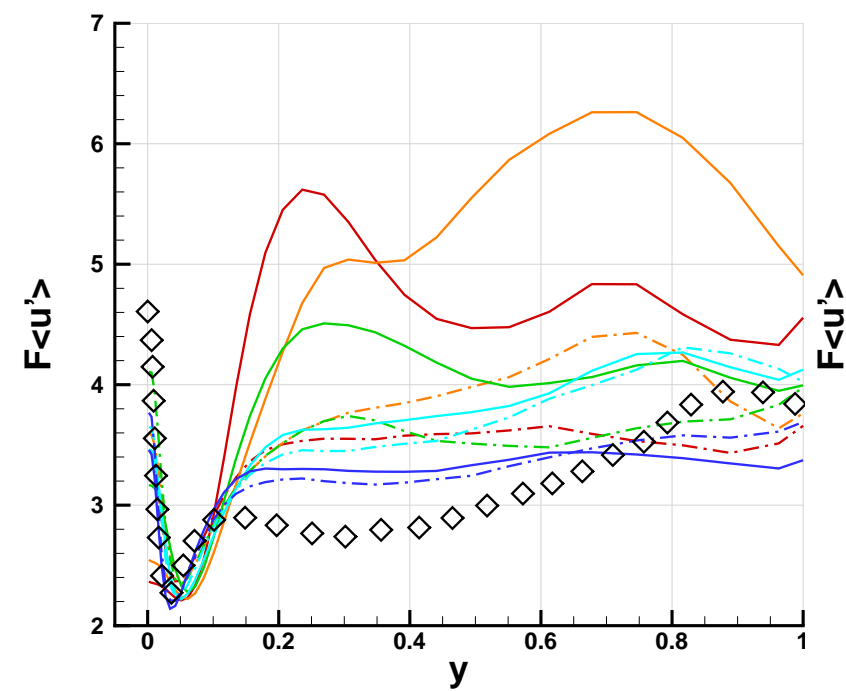

(a) $64^{3}$

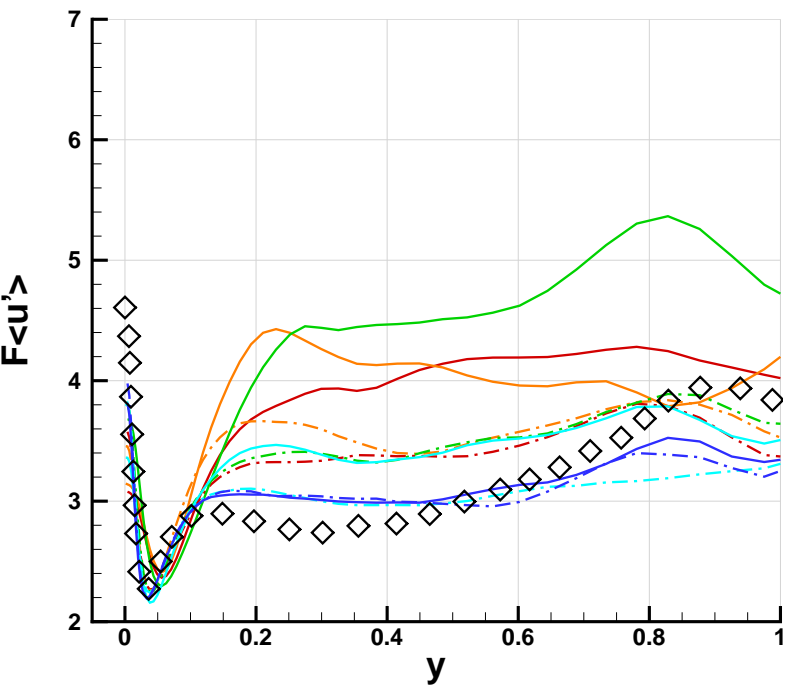

(b) $96^{3}$

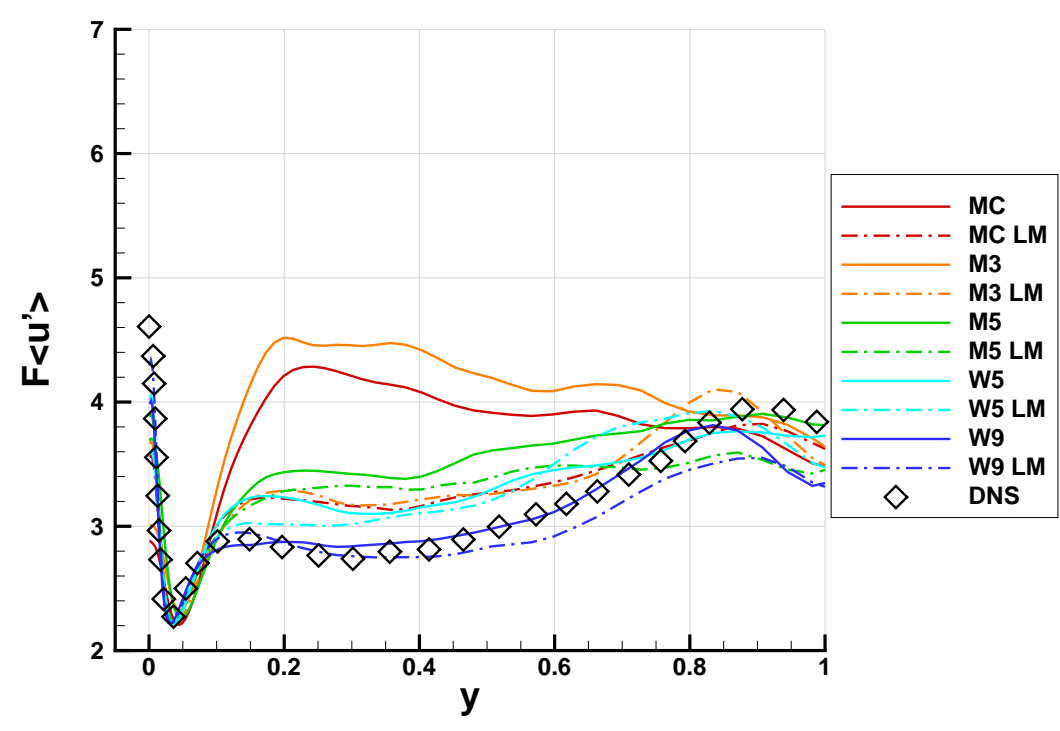

(c) $128^{3}$

Figure 13: Flatness of the streamwise velocity 


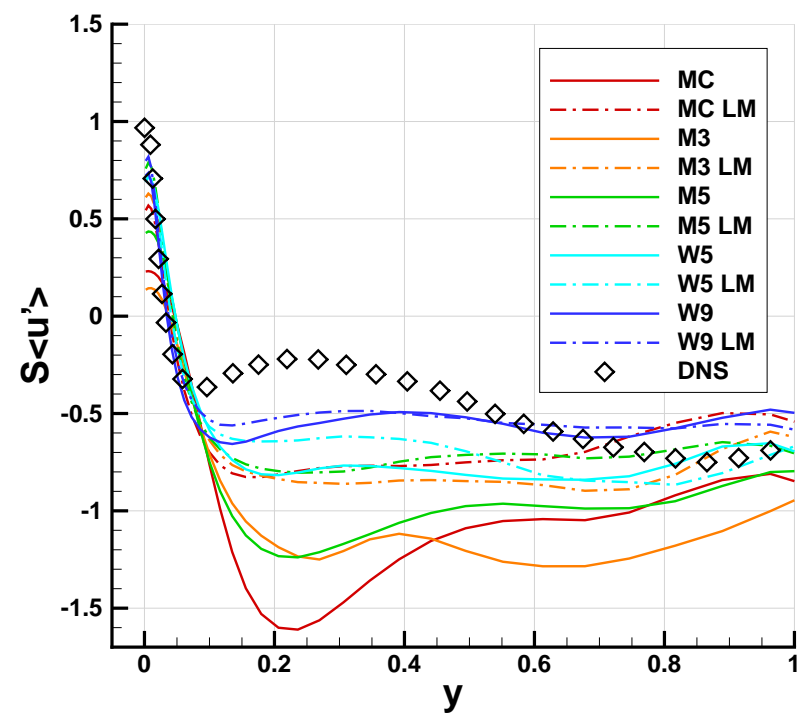

(a) $64^{3}$

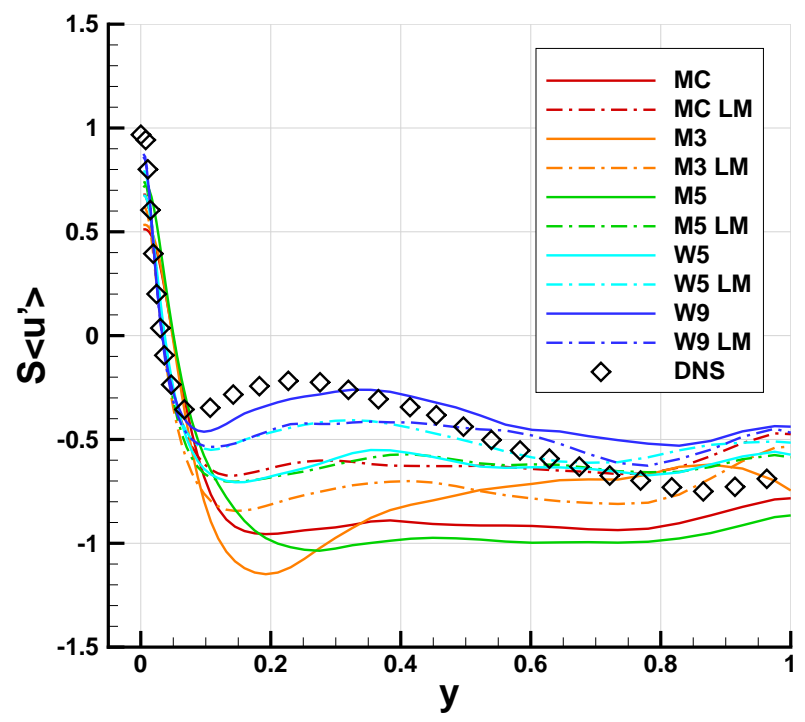

(b) $96^{3}$

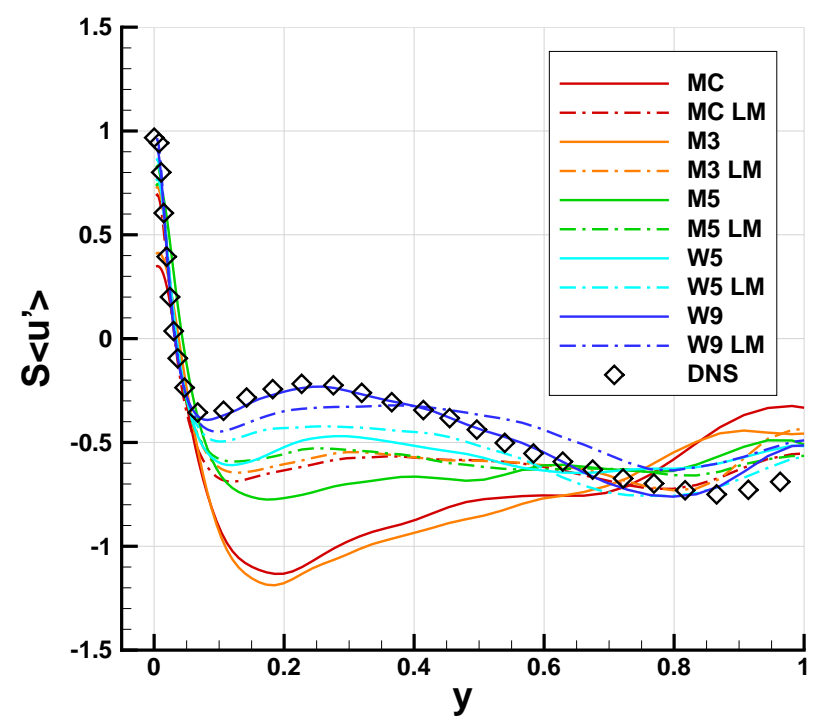

(c) $128^{3}$

Figure 14: Skewness of the streamwise velocity 


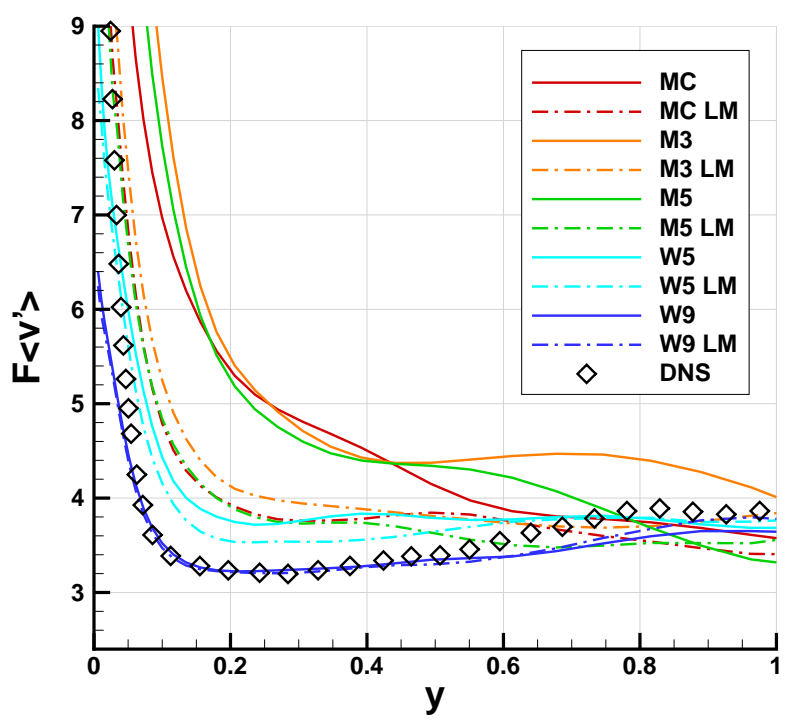

(a) $64^{3}$

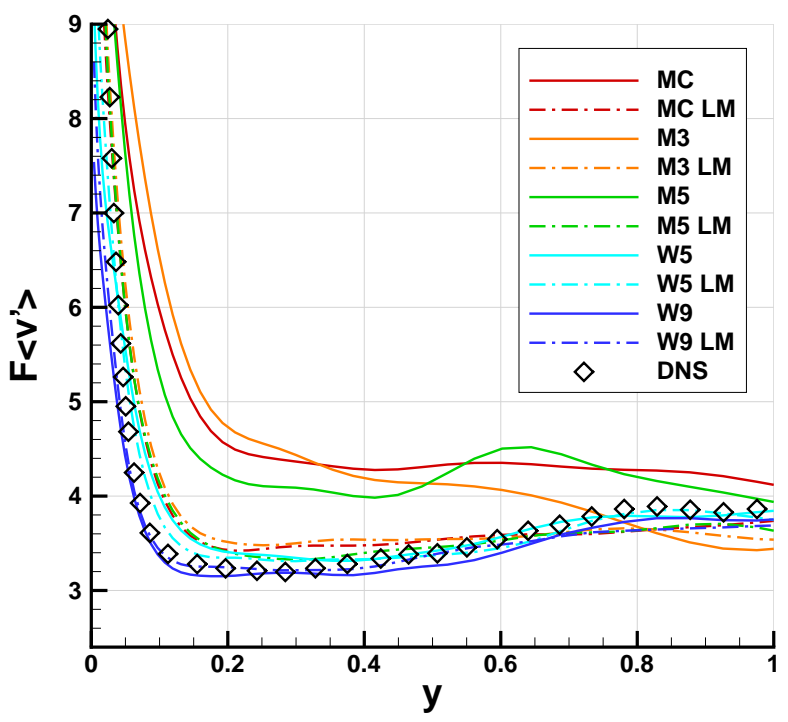

(b) $96^{3}$

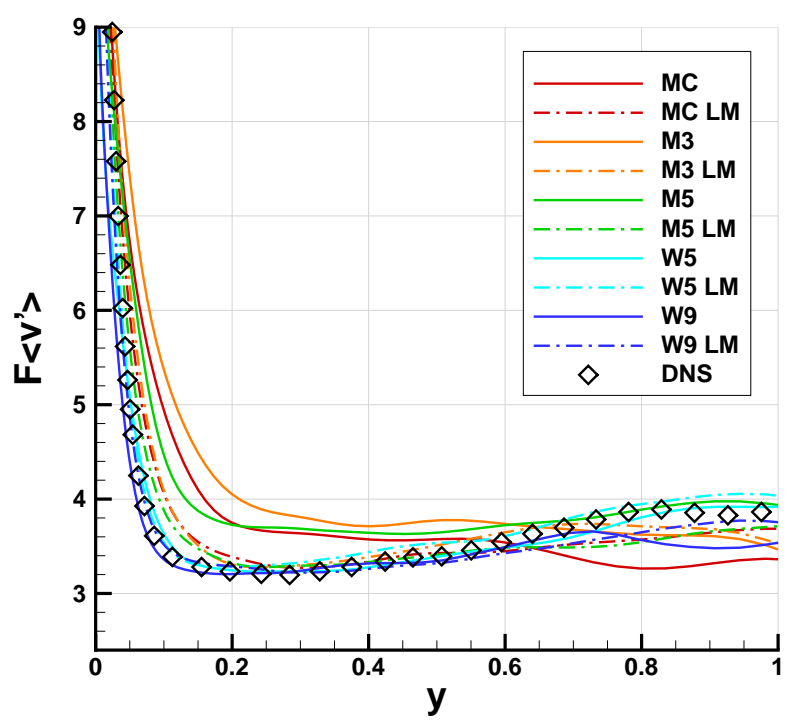

(c) $128^{3}$

Figure 15: Flatness of the wall-normal velocity 


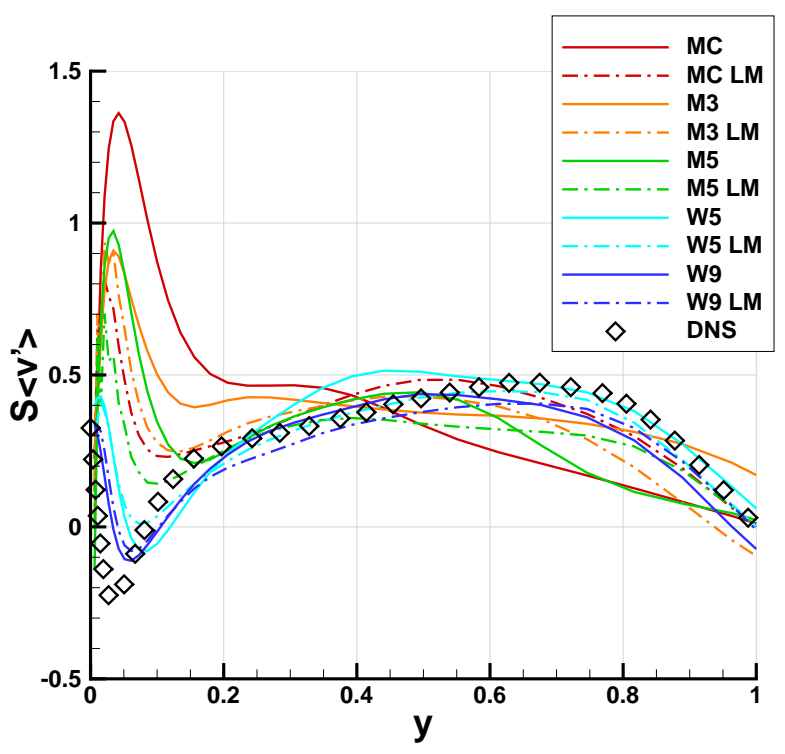

(a) $64^{3}$

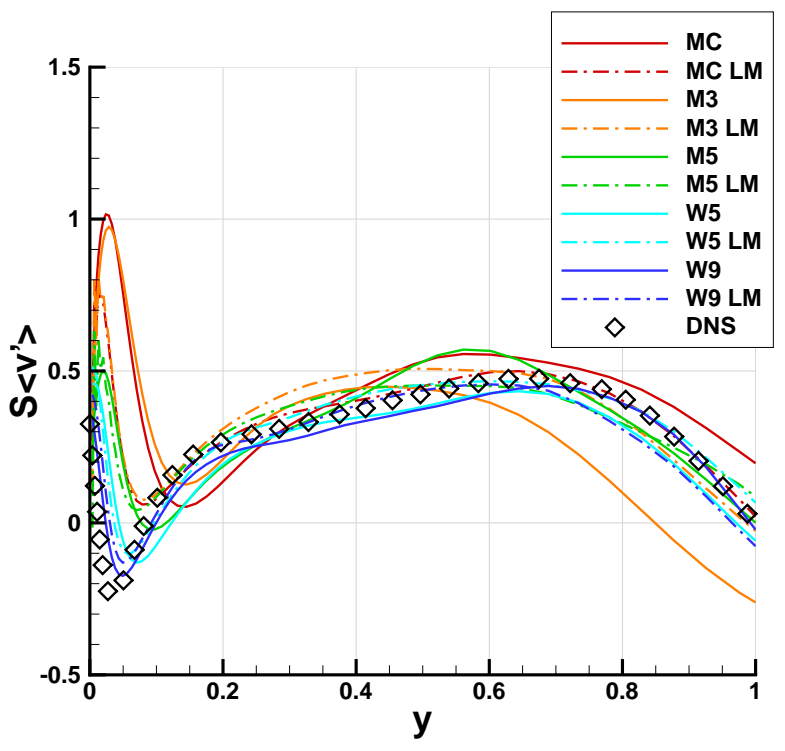

(b) $96^{3}$

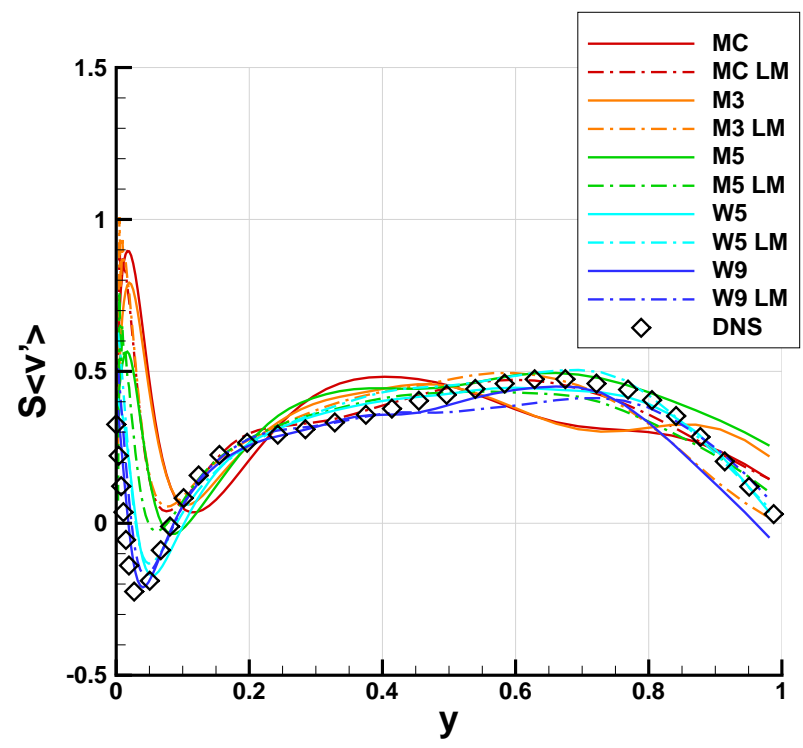

(c) $128^{3}$

Figure 16: Skewness of the wall-normal velocity 


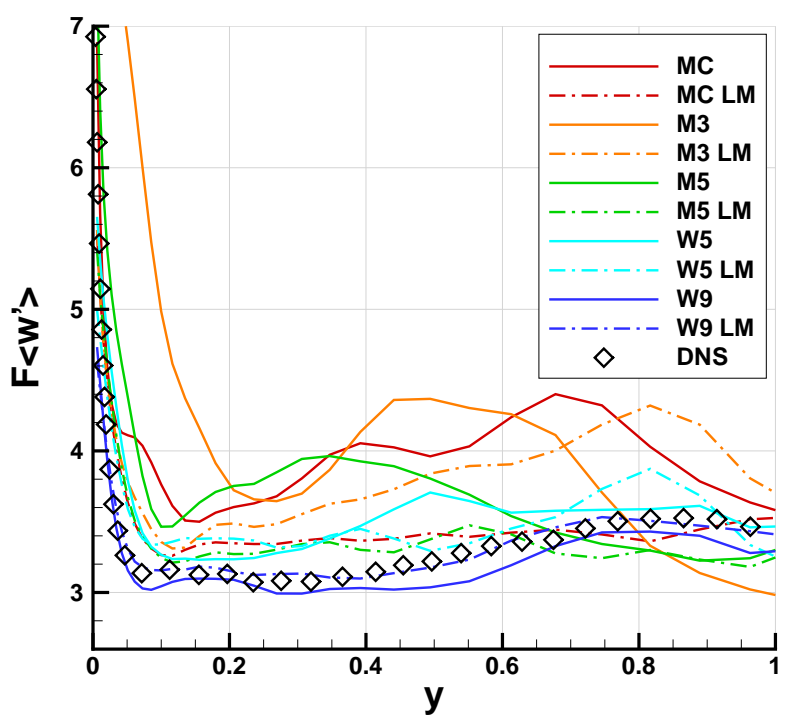

(a) $64^{3}$

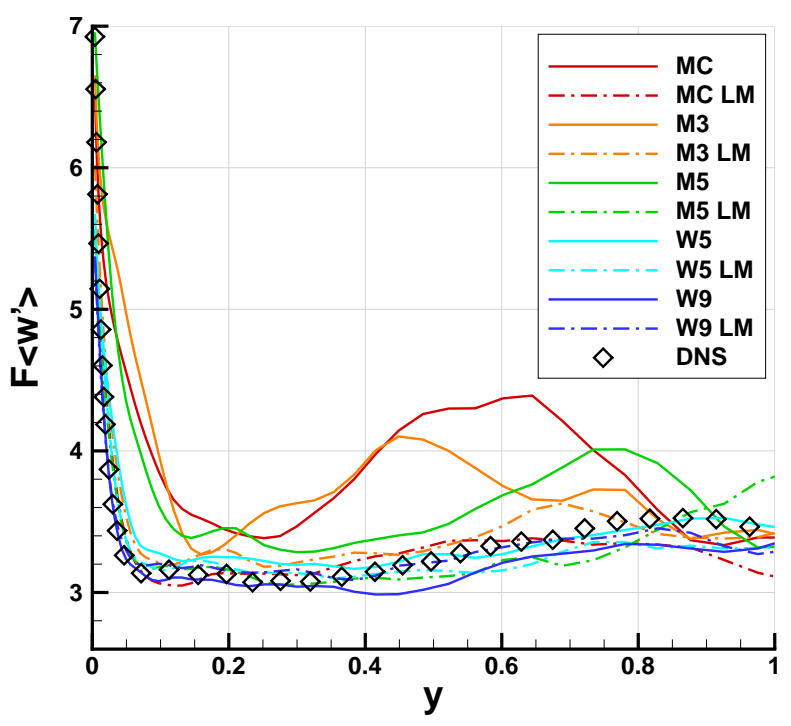

(b) $96^{3}$

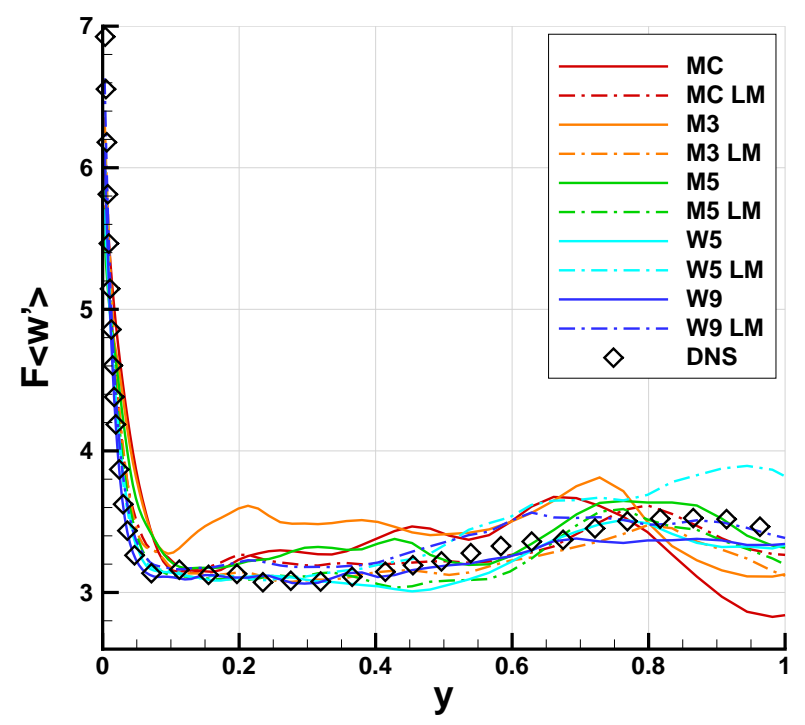

(c) $128^{3}$

Figure 17: Flatness of the spanwise velocity 


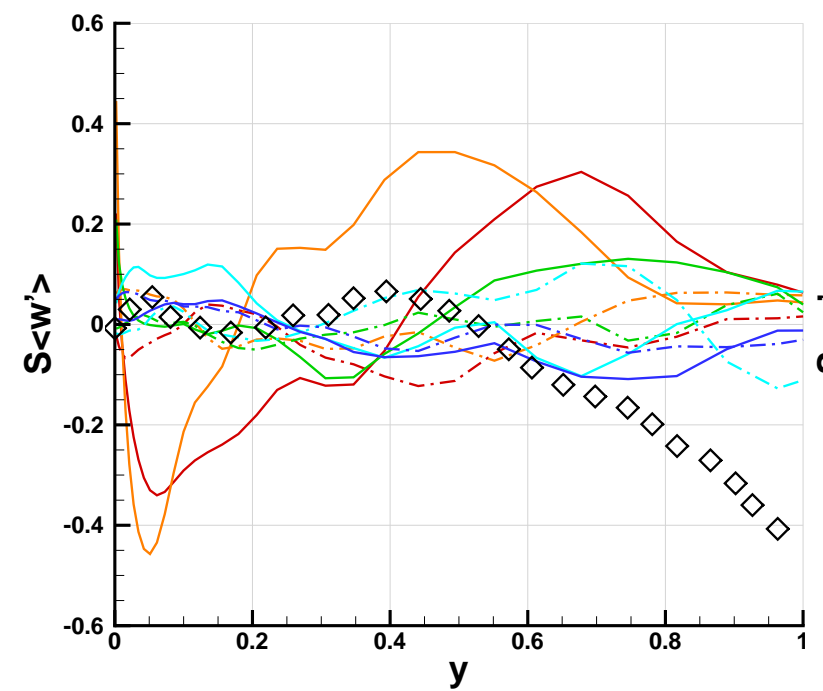

(a) $64^{3}$

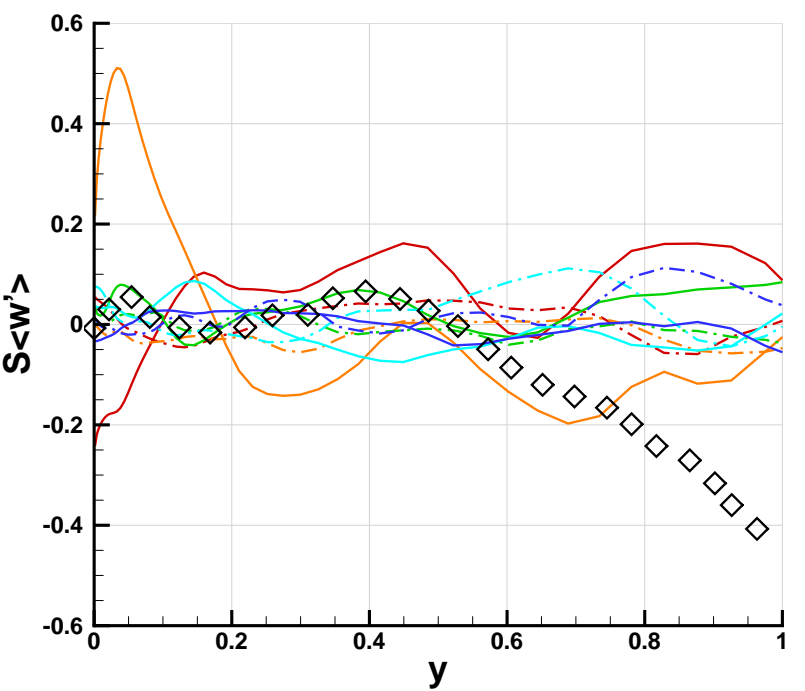

(b) $96^{3}$

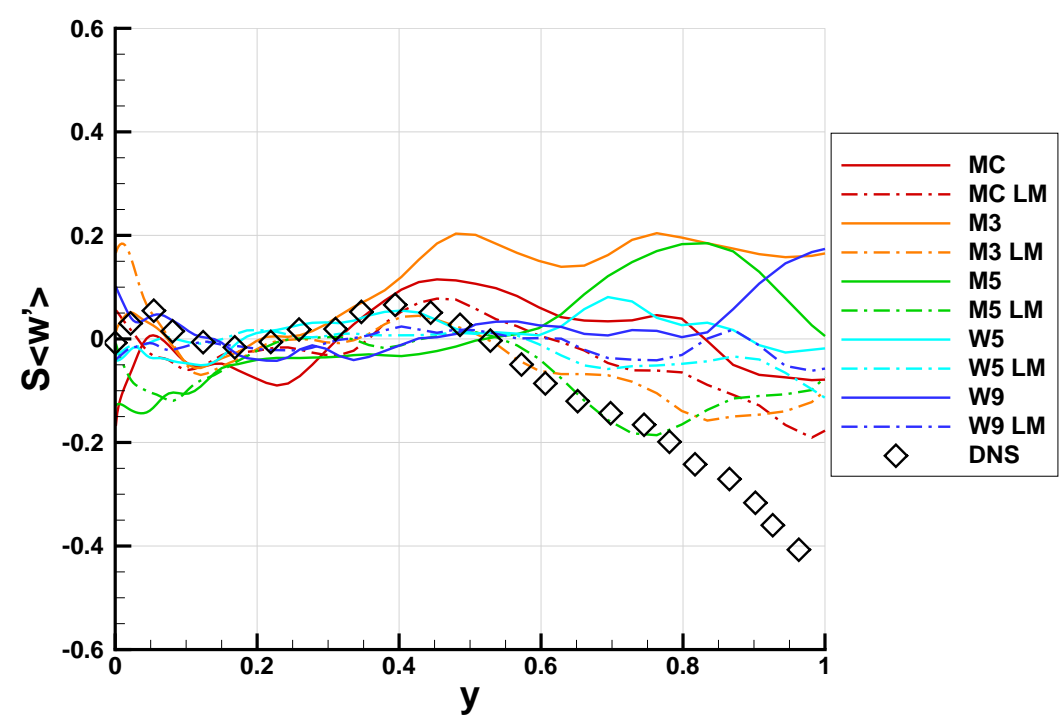

(c) $128^{3}$

Figure 18: Skewness of the spanwise velocity 


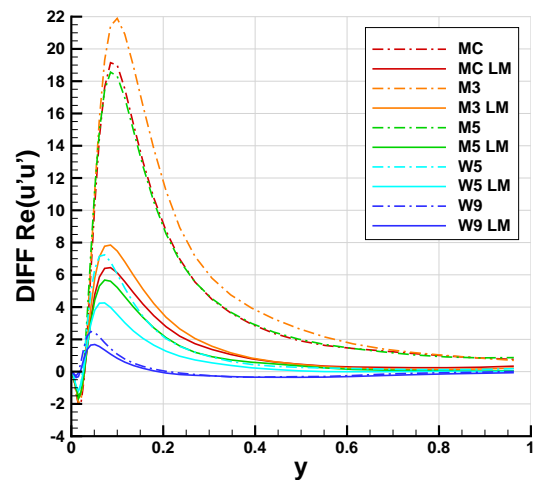

(a) $64^{3}$

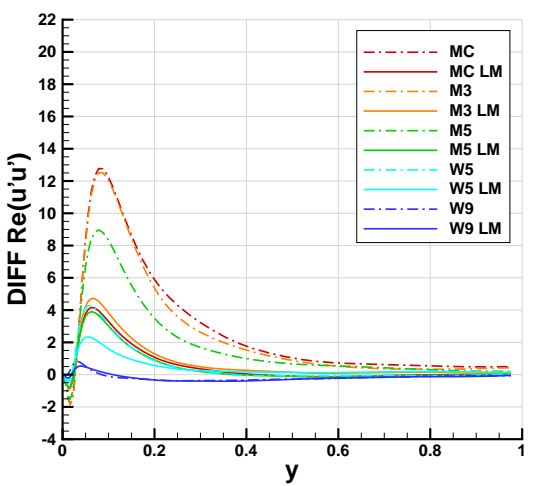

(b) $96^{3}$

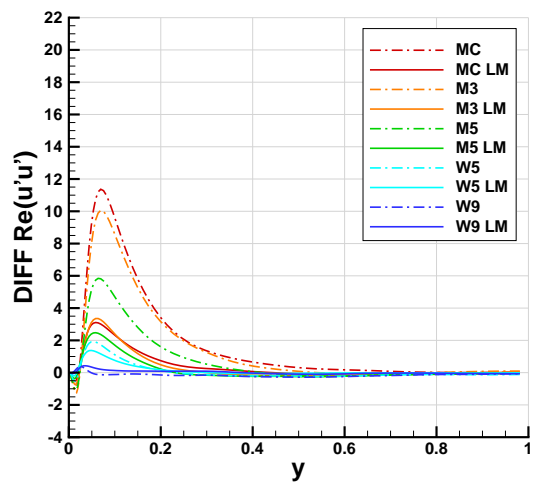

(c) $128^{3}$

Figure 19: Grid convergence for streamwise velocity stresses $\operatorname{Re}\left(u^{\prime} u^{\prime}\right)$

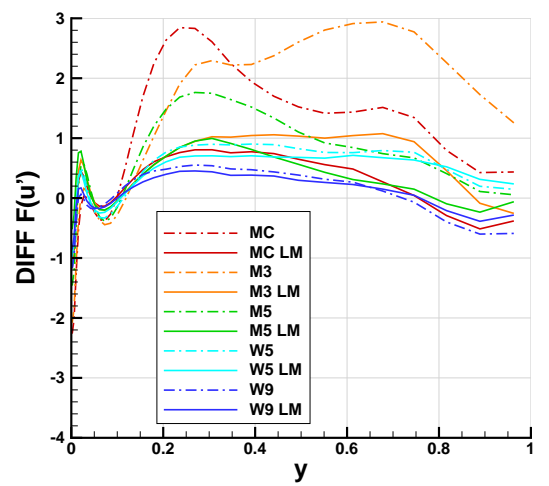

(a) $64^{3}$

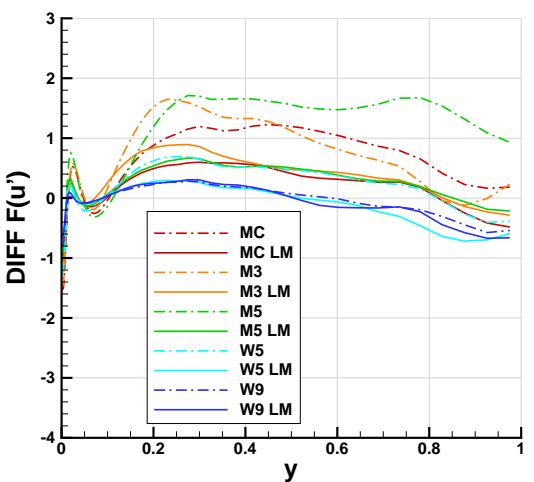

(b) $96^{3}$

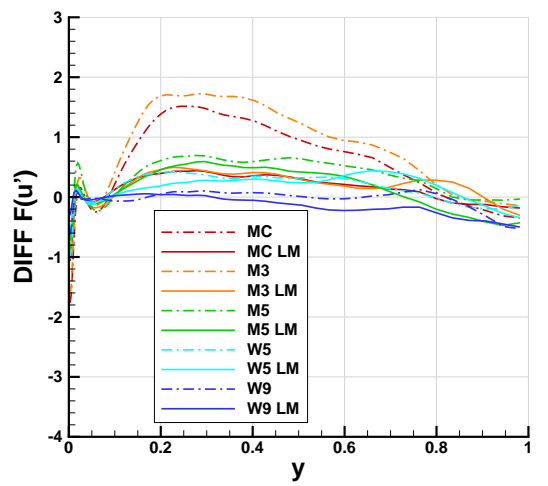

(c) $128^{3}$

Figure 20: Grid convergence for streamwise flatness $F\left(u^{\prime}\right)$

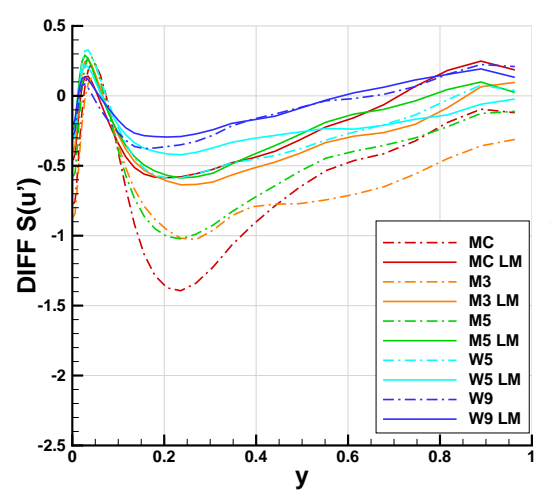

(a) $64^{3}$

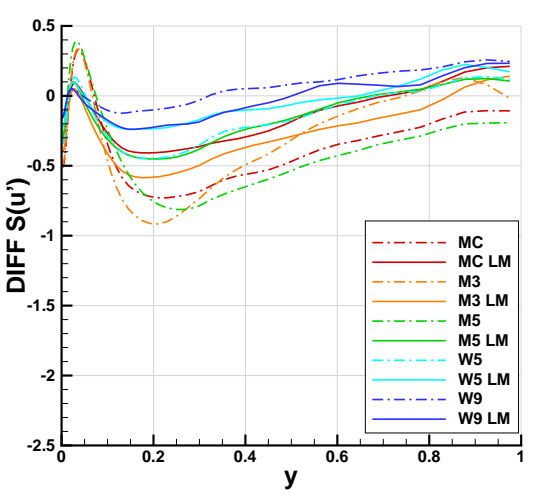

(b) $96^{3}$

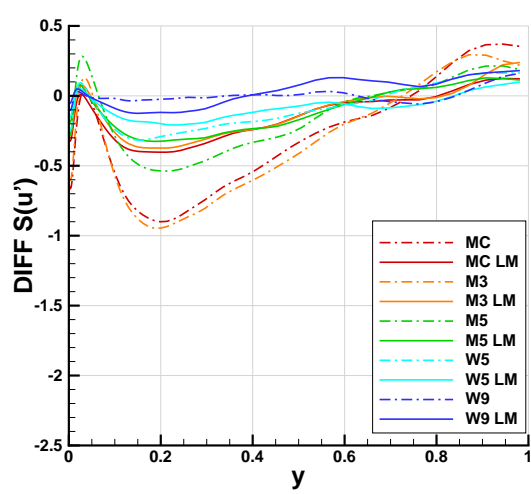

(c) $128^{3}$

Figure 21: Grid convergence for streamwise skewness $S\left(u^{\prime}\right)$ 


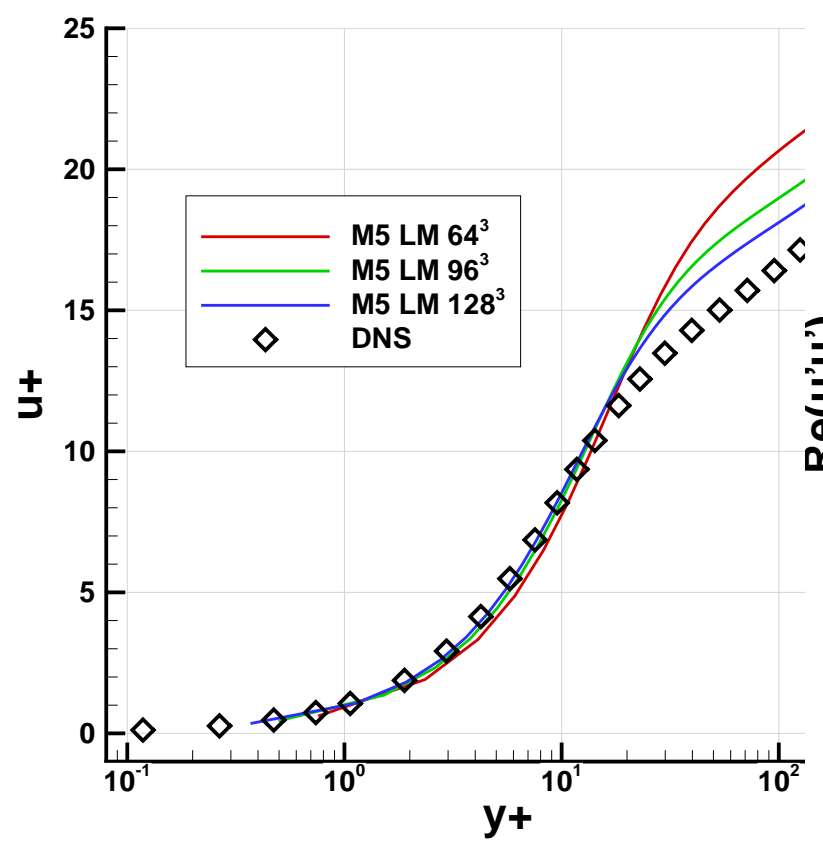

(a) $u+$ vs. $y+$

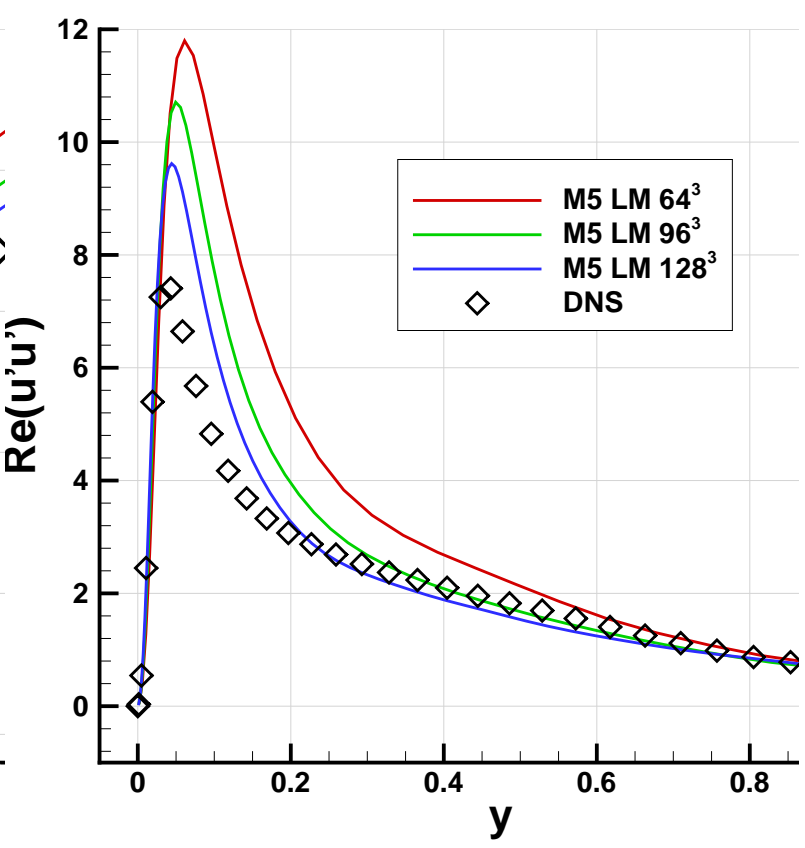

(b) $\operatorname{Re}\left(u^{\prime} u^{\prime}\right)$ vs. $y$

Figure 22: Grid convergence for MUSCL $5^{\text {th }}$ with LM correction

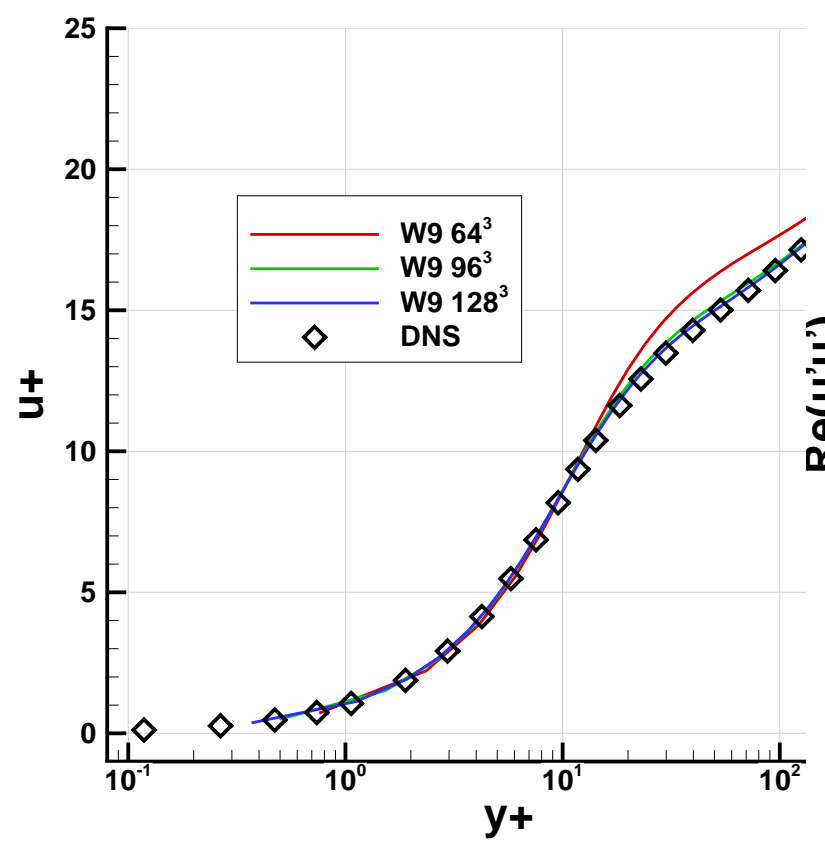

(a) $u+$ vs. $y+$

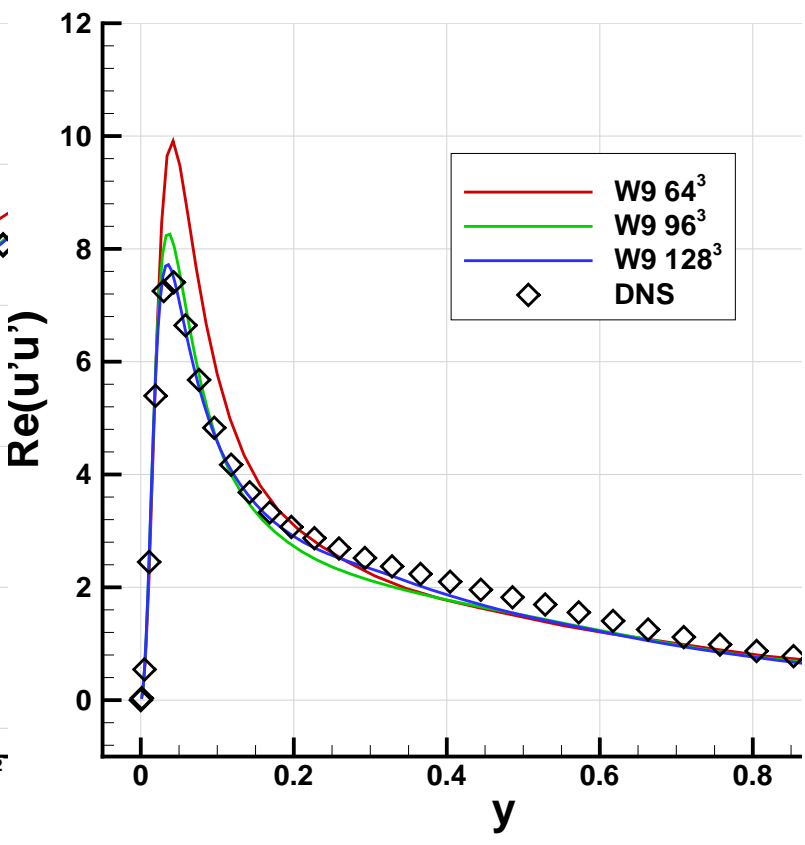

(b) $\operatorname{Re}\left(u^{\prime} u^{\prime}\right)$ vs. $y$

Figure 23: Grid convergence for WENO $9^{\text {th }}$ 


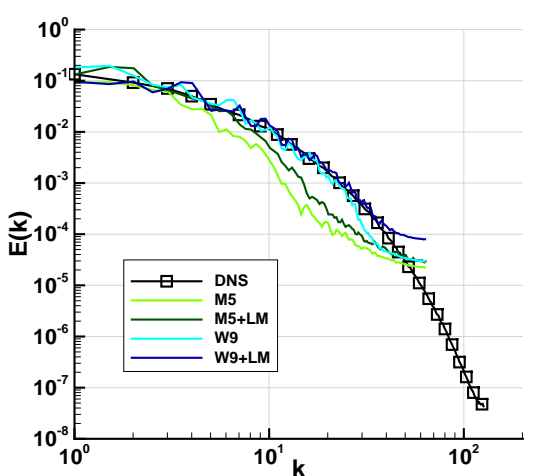

(a) Streamwise velocity

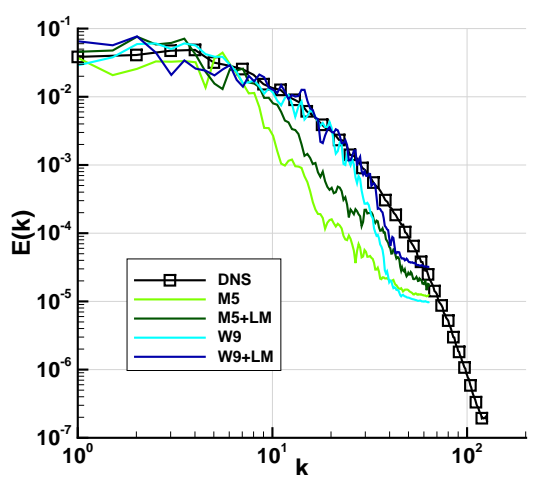

(b) Wall-normal velocity

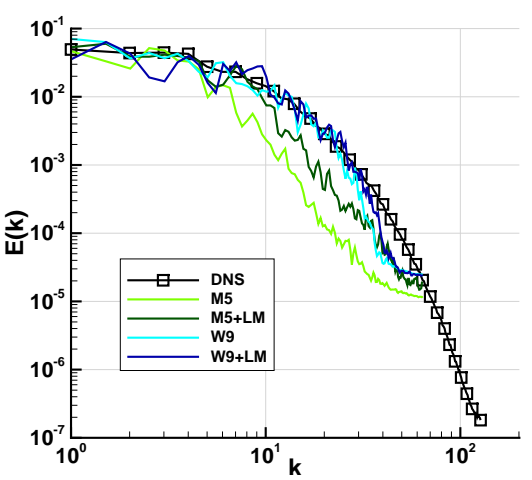

(c) Spanwise velocity

Figure 24: Energy Spectra on $128^{3}$ grid at $y^{+}=395$ in streamwise direction

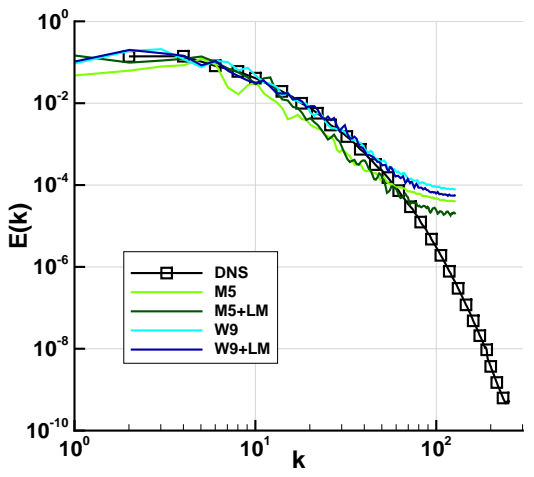

(a) Streamwise velocity

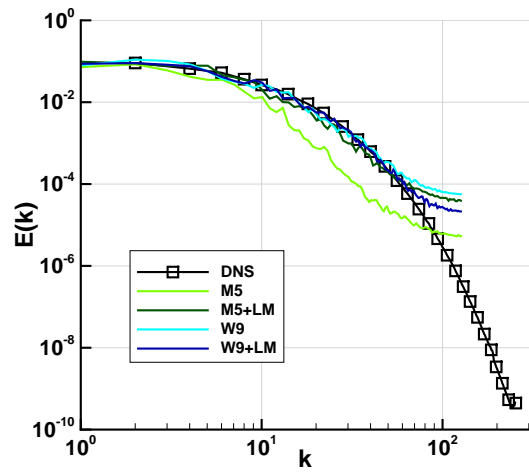

(b) Wall-normal velocity

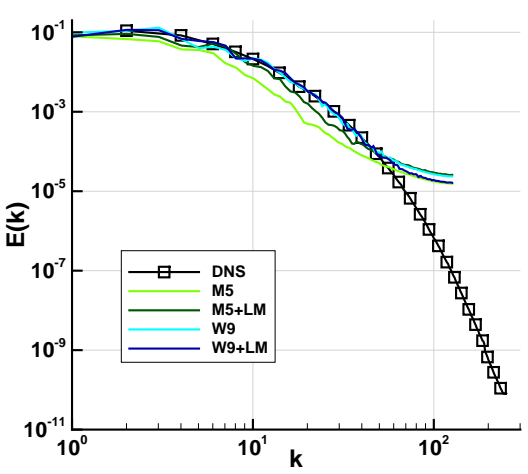

(c) Spanwise velocity

Figure 25: Energy Spectra on $128^{3}$ grid at $y^{+}=395$ in spanwise direction

correction on the W9 scheme at larger wavenumbers agree with previous computational evidence from DNS databases [127-130] suggesting that the interactions between large resolved scales and unresolved scales are less significant than those between small resolved scales and unresolved scales.

By integrating the ILES and DNS energy spectra an estimate of the total resolved energy can be obtained. A quantitative comparison of the spectra results for DNS, MUSCL $5^{\text {th }}$ and WENO $9^{\text {th }}$ is presented in Table 2 and Table 3 for the total resolved energy. The WENO $9^{\text {th }}$ order gives results closer to DNS than the MUSCL $5^{\text {th }}$. The LM correction consistently improves the accuracy of the MUSCL $5^{\text {th }}$ order, however, its effects are more ambiguous with respect to WENO $9^{\text {th }}$ order.

The domain size was chosen to be sufficiently large to contain all turbulent scales resolved and it is thus expected that the temporal variability of the resolved friction Reynolds number should remain low. An indication of the above is provided by calculating the standard deviation of the resolved friction Reynolds number $\sigma\left(\operatorname{Re}_{\tau}\right)$ over the total number of samples $\left(N_{s}\right)$ used to obtain the averaged statistical profiles:

$$
\sigma\left(R e_{\tau}\right)=\sqrt{\frac{1}{N_{s}} \sum_{n=1}^{N_{s}}\left[\operatorname{Re}_{\tau}(n)-\overline{R e}_{\tau}\right]^{2}}
$$

where $\operatorname{Re}_{\tau}(n)=\bar{\rho} u_{\tau} \delta / \bar{\mu}$ is the resolved friction Reynolds number at time-step $n$, and $\overline{R e}_{\tau}$ is the ensemble average of 


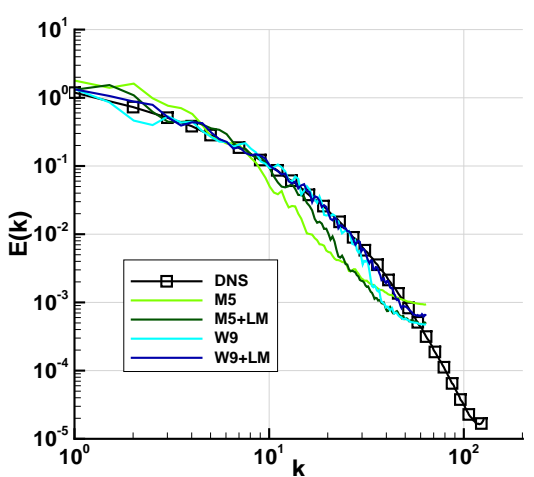

(a) Streamwise velocity

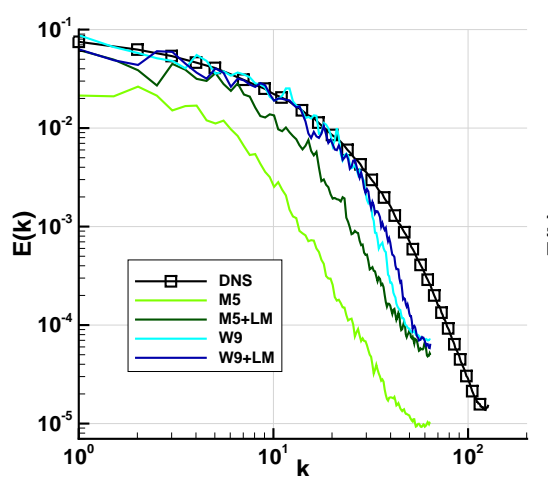

(b) Wall-normal velocity

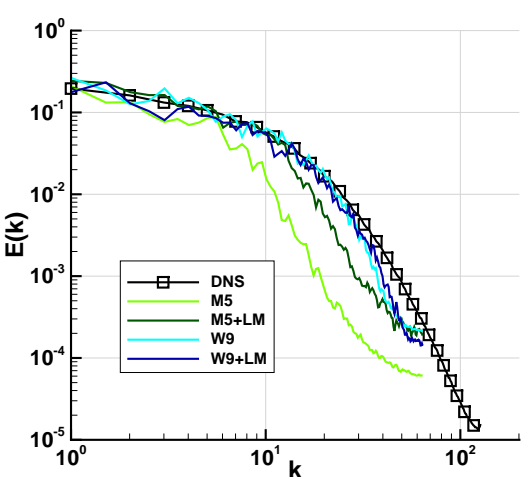

(c) Spanwise velocity

Figure 26: Energy Spectra on $128^{3}$ grid at $y^{+}=30$ in streamwise direction

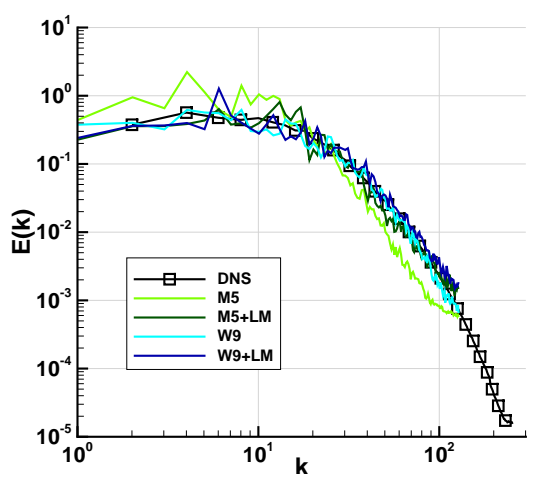

(a) Streamwise velocity

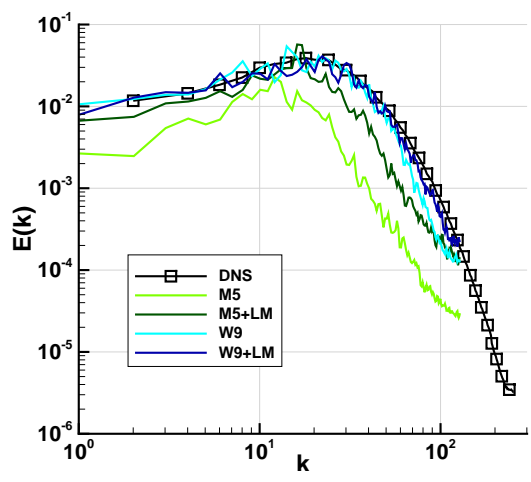

(b) Wall-normal velocity

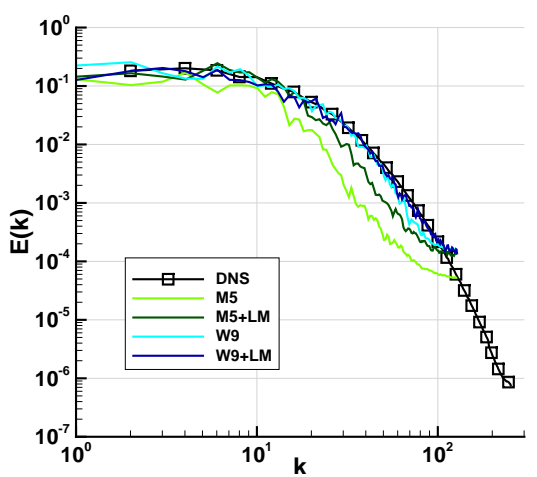

(c) Spanwise velocity

Figure 27: Energy Spectra on $128^{3}$ grid at $y^{+}=30$ in spanwise direction 


\begin{tabular}{|c|ccc|}
\hline $\begin{array}{c}\text { Streamwise } \\
\text { Spectrum }\end{array}$ & $\begin{array}{c}\text { Streamwise } \\
\text { Velocity }\end{array}$ & $\begin{array}{c}\text { Wall-normal } \\
\text { Velocity }\end{array}$ & $\begin{array}{c}\text { Spanwise } \\
\text { Velocity }\end{array}$ \\
\hline MUSCL 5 $^{\text {th }}$ & 7.132 & 0.138 & 0.821 \\
MUSCL 5 $^{\text {th }}+\mathbf{L M}$ & 5.310 & 0.376 & 1.409 \\
WENO 9 $^{\text {th }}$ & 4.873 & 0.636 & 1.612 \\
WENO 9 $^{\text {th }}+\mathbf{L M}$ & 5.385 & 0.580 & 1.395 \\
DNS $^{\text {Spanwise }}$ & 5.105 & 0.675 & 1.593 \\
\hline Spectrum $^{\text {Streamwise }}$ & Wall-normal & Spanwise \\
\hline MUSCL 5 $^{\text {th }}$ & 17.193 & 0.302 & 1.594 \\
MUSCL 5 $^{\text {th }}+\mathbf{L M}$ & 11.891 & 0.776 & 2.774 \\
WENO 9 $^{\text {th }}$ & 10.810 & 1.245 & 3.118 \\
WENO 9 $^{\text {th }}+\mathbf{L M}$ & 11.607 & 1.204 & 2.788 \\
DNS $^{\text {DUN }}$ & 11.241 & 1.386 & 3.189 \\
\hline
\end{tabular}

Table 2: Total resolved Energy Spectra near the wall $\left(y^{+} \approx 30\right)$

\begin{tabular}{|c|ccc|}
\hline $\begin{array}{c}\text { Streamwise } \\
\text { Spectrum }\end{array}$ & $\begin{array}{c}\text { Streamwise } \\
\text { Velocity }\end{array}$ & $\begin{array}{c}\text { Wall-normal } \\
\text { Velocity }\end{array}$ & $\begin{array}{c}\text { Spanwise } \\
\text { Velocity }\end{array}$ \\
\hline MUSCL 5 $^{\text {th }}$ & 0.333 & 0.235 & 0.213 \\
MUSCL 5 $^{\text {th }}+\mathbf{L M}$ & 0.543 & 0.369 & 0.304 \\
WENO 9 $^{\text {th }}$ & 0.675 & 0.404 & 0.375 \\
WENO 9 $^{\text {th }}+\mathbf{L M}$ & 0.573 & 0.408 & 0.382 \\
DNS $^{\text {Spanwise }}$ & 0.572 & 0.406 & 0.399 \\
Spectrum $^{\text {Streamwise }}$ & Wall-normal & Spanwise \\
\hline MUSCL 5 $^{\text {th }}$ & 0.690 & 0.440 & 0.392 \\
MUSCL 5 $^{\text {th }}+\mathbf{L M}$ & 1.111 & 0.718 & 0.573 \\
WENO 9 $^{\text {th }}$ & 1.326 & 0.763 & 0.712 \\
WENO 9 $^{\text {th }}+\mathbf{L M}$ & 1.232 & 0.735 & 0.728 \\
DNS $^{\text {DUNity }}$ & 1.187 & 0.773 & 0.754 \\
\hline
\end{tabular}

Table 3: Total resolved Energy Spectra near the midstream $\left(y^{+} \approx 392\right)$ 


\begin{tabular}{|c|cc|cc|}
\hline Grid: 64 $^{3}$ & $\overline{R e}_{\tau}$ & $\sigma\left(R e_{\tau}\right)$ & $\mathbf{L M :} \overline{R e}_{\tau}$ & $\mathbf{L M : ~} \sigma\left(R e_{\tau}\right)$ \\
\hline MC 2 2 $^{\text {nd }}$ & 279.60 & 10.35 & 320.47 & 5.51 \\
MUSCL 3 $^{\text {rd }}$ & 269.90 & 8.78 & 309.78 & 6.16 \\
MUSCL 5 $^{\text {th }}$ & 277.85 & 6.76 & 322.20 & 3.90 \\
WENO 5 $^{\text {th }}$ & 315.87 & 5.72 & 331.37 & 3.51 \\
WENO 9 9 $^{\text {th }}$ & 372.17 & 3.64 & 367.58 & 2.91 \\
\hline Grid: 96 $^{3}$ & & & & \\
\hline MC 2 2 $^{\text {nd }}$ & 292.97 & 9.25 & 343.77 & 4.19 \\
MUSCL 3 $^{\text {rd }}$ & 290.05 & 8.02 & 342.21 & 3.33 \\
MUSCL 5 5 $^{\text {th }}$ & 304.34 & 5.81 & 349.28 & 4.24 \\
WENO 5 5 $^{\text {th }}$ & 343.24 & 3.91 & 360.83 & 3.96 \\
WENO 9 $^{\text {th }}$ & 387.70 & 3.53 & 382.11 & 3.02 \\
\hline${\text { Grid: } 128^{3}}^{3}$ & & & \\
\hline MC 2 2d & 309.14 & 7.16 & 359.10 & 4.45 \\
MUSCL 3 $^{\text {rd }}$ & 308.26 & 7.02 & 355.14 & 3.08 \\
MUSCL 5 $^{\text {th }}$ & 325.65 & 2.88 & 366.61 & 2.24 \\
WENO 5 $^{\text {th }}$ & 360.85 & 2.75 & 374.75 & 2.96 \\
WENO 9 $^{\text {th }}$ & 393.13 & 1.85 & 386.60 & 2.28 \\
\hline
\end{tabular}

Table 4: Resolved friction Reynolds numbers $\left(\overline{\operatorname{Re}}_{\tau}\right)$ and standard deviation $(\sigma)$

the resolved friction Reynolds number over the entire sample range:

$$
\overline{R e}_{\tau}=\frac{1}{N_{s}} \sum_{n=1}^{N_{s}} \operatorname{Re}_{\tau}(n)
$$

The standard deviation $(\sigma)$ is indicative of the ability of high-order scheme to sustain a turbulent field; lower values attained suggest greater accuracy and lower intermittency. The $\sigma$ values obtained for all configurations examined herein are summarized in Table 4 along with their resolved friction Reynolds number. W9 encompasses the lowest value out of all high-order schemes examined, with a $\sigma$ value of below 4 for all mesh resolutions, with or without LM correction. On the other hand, the $2^{\text {nd }}$-order MUSCL scheme (MC) showed the greatest variability with a value as high as 10 on the coarse grid. Apart from the WENO schemes on the fine grid $\left(128^{3}\right)$, the use of the LM correction led to significant reductions of $\sigma$, especially for the lower-order MUSCL schemes. Concerning the resolved friction Reynolds number, the LM correction improves the ILES results of the MUSCL schemes by 10-20\%.

The computational requirements in LES is another important issue and will remain so in the foreseeable future despite the advancements in high-performance computing. To analyse the computational efficiency of each numerical scheme at different grid resolutions, the results of Table 4 are used to establish the error reduction versus computational cost. The numerical error, $\varepsilon_{N}$, is estimated by the average resolved friction Reynolds number $\left(\overline{R e}_{\tau}\right)$ of Equation (4):

$$
\varepsilon_{N}=\frac{395-\overline{R e}_{\tau}}{395} \times 100 \%
$$

with the target reference friction Reynolds number being 395. Note that the DNS of [30] reported a resolved friction 


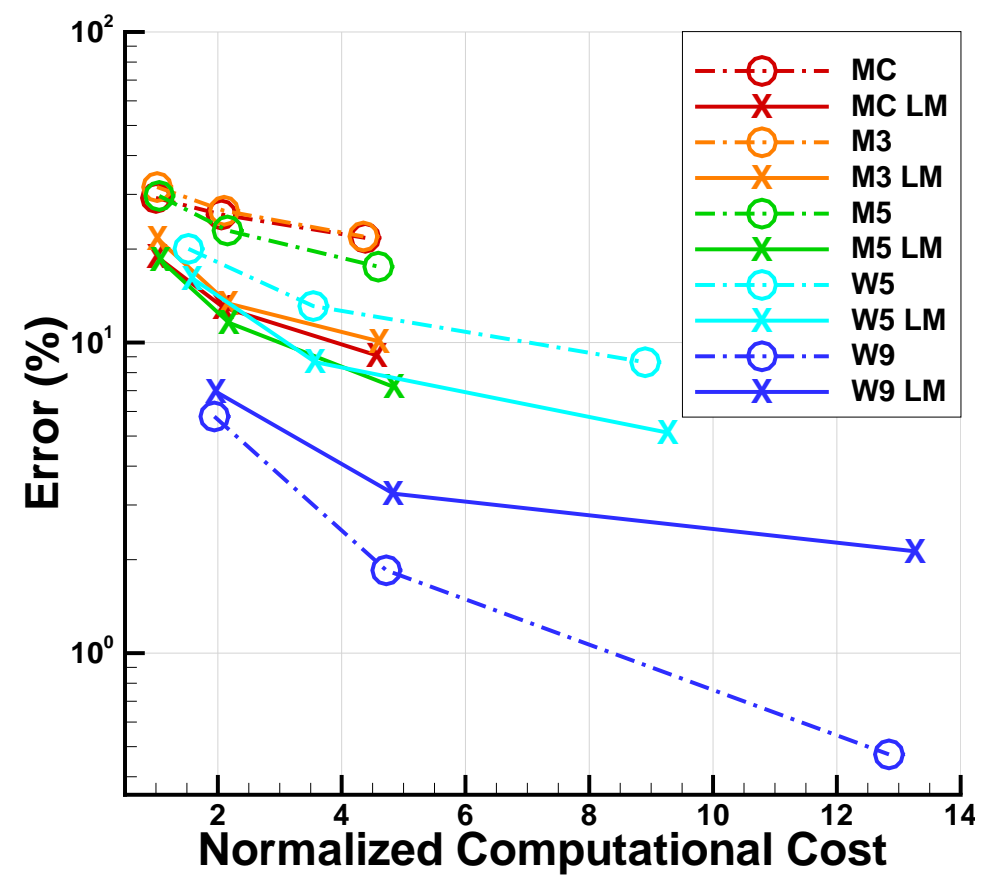

Figure 28: Error vs. normalized computational cost

Reynolds number of $R e_{\tau}^{D N S}=392.24$.

The computational cost is estimated using as reference the fastest simulation, namely the $2^{\text {nd }}$-order MC scheme without the LM correction on the coarse $64^{3}$ grid. Dividing the computational time of each simulation by the reference value, a normalised computational time is obtained.

The obtained error $\left(\varepsilon_{N}\right)$ vs computational cost for each scheme is plotted in Figure 28. The $128^{3}$ grid contains exactly 8 times more cells than the coarse grid and 2.4 time than the medium grid, thus the computational time is expected to increase accordingly. The WENO schemes are more accurate than the MUSCL ones, but at the same time are much more computationally intensive. Note that due to the simplicity of the LM correction [86], its overhead in the total simulation time is almost negligible as seen in Figure 28. The W9 scheme is approximately 1.4 to 2.7 times more expensive than the W5 and M5 schemes, respectively. However, the WENO schemes enable the simulations to approach the target DNS value at much coarser meshes. The W9 scheme on the $64^{3}$ grid was capable of obtaining a friction Reynolds number that was closer to the target value of 395 than any of the remaining schemes, even when used on a finer grid $\left(128^{3}\right)$ in conjunction with the LM correction. Specifically, the W9 scheme on the coarse grid $\left(64^{3}\right)$ is approximately as computationally expensive as any of the MUSCL schemes are on the medium grid $\left(96^{3}\right)$ for approximately half the error. For example, the coarse grid ILES using W9 gives better results than the fine grid ILES using M5 and LM correction for half of the computational cost. As a final remark, we should note that the analysis above should be considered by taking into account that there is an uncertainty associated with the DNS and that if one accounts for the error bounds in the DNS solution (with reference to the experiment), then this might elucidate the error associated with the W9 scheme with and without the LM correction.

\section{Conclusions}

A detailed investigation of the accuracy and efficiency of high-order MUSCL and WENO schemes in the framework of ILES has been conducted for a weakly-compressible, fully-developed, turbulent channel flow. The study has led to useful insights with respect to the effects of these schemes on the accuracy of ILES in wall-bounded turbulent flows, and a summary of the key conclusions is provided below. 
The LM correction [87] significantly improves the accuracy of the simulations, yielding results on coarse grids that are as accurate as those obtained on finer grids of twice the size. On the finer mesh, the extent of the improvement for all MUSCL schemes is such that the solutions compare well to data obtained by DNS for a wide range of the statistical quantities investigated.

The LM correction can significantly reduce the numerical dissipation intrinsic to shock-capturing methods at relatively low Mach numbers, leading to a drastic improvement in the resolution and capturing of turbulent structures. As a consequence, the turbulent boundary layer statistics appear to converge towards those obtained by DNS, suggesting that the LM correction leads to physically correct results when applied to flows with no-slip walls. Most importantly, this is accomplished at a very small computational overhead. The effects of the LM correction appear to be greater when it is implemented in conjunction with second- and third-order methods. The fifth-order MUSCL and WENO schemes also benefit from the LM correction, with the WENO $5^{\text {th }}$-order to a lesser degree.

The ninth-order WENO (W9) scheme gives by far the most accurate results out of all the schemes investigated in this study. The results obtained by W9 closely match the DNS data, with some noticeable differences appearing only for the higher order statistical quantities of flatness and skewness on the coarse grid. The W9 demonstrated to be capable of accurately resolving practically all statistical quantities examined without requiring the LM correction even on the coarsest grid. The high-order statistics W9 results suggested that the inclusion of the LM correction may adversely affect the accuracy of its results.

It can be argued that the order of the leading even-order truncation error term of high-order schemes such as WENO $5^{\text {th }}$ is greater than two [131] and therefore does not match the order of the dissipation differential form found in turbulence models. However, in [132] it was shown that for a successful ILES scheme, the nonlinear dissipation must be either of the same form as that observed experimentally and also derived mathematically in turbulence theory, or otherwise have minimal interference with the terms associated with conservation form. Consequently, the ninth-order scheme is found to have the least numerical dissipation out of all schemes employed and for this reason performed better even on the coarse grid. No other scheme was capable of resolving the near-wall region so adequately despite the use of low-Mach corrections and finer meshes.

The increased accuracy and intrinsic low Mach number dissipation properties of W9 come at an increased computational cost. This is the main disadvantage of the W9 scheme. Nonetheless, its accuracy (with respect to the target friction Reynolds number) even on the coarse grid was stellar, just under $6 \%$, whereas the best result obtained by the fine grid simulations using the M5 scheme in conjunction with the LM correction was slightly over 7\%. The M5 with LM correction on the fine grid required more than twice the computational time compared to the W9 on the coarse grid and, additionally, the W9 gave significantly more accurate results.

The energy spectra suggest that inadequate resolution of the near wall region leads to a reduction in accuracy of the resolved Reynolds stresses and higher order statistics. High-order numerical methods such as W9 are naturally less dissipative and offer the potential of adequately resolving a wider range of the energy spectrum, almost up until the grid cut-off wavenumber. At this point, the problem is not just the amount of numerical dissipation, but also the backscatter-like accumulation of the turbulent kinetic energy at the near cut-off wavenumbers due to dispersive (odd-order truncation terms) errors, similar to unresolved spectral simulations.

\section{References}

[1] J. Boris, F. Grinstein, E. Oran, R. Kolbe, New insights into large eddy simulation, Fluid Dynamics Research 10 (46) (1992) 199 - 228, doi:10.1016/0169-5983(92)90023-P.

[2] C. Hirt, Heuristic stability theory for finite-difference equations, Journal of Computational Physics 2 (4) (1968) 339-355, ISSN 0021-9991, doi:10.1016/0021-9991(68)90041-7.

[3] J. A. Domaradzki, S. Radhakrishnan, Effective eddy viscosities in implicit modeling of decaying high Reynolds number turbulence with and without rotation, Fluid Dynamics Research 36 (46) (2005) 385-406, doi:10.1016/j.fluiddyn.2004.08.004.

[4] L. G. Margolin, W. J. Rider, A rationale for implicit turbulence modelling, International Journal for Numerical Methods in Fluids 39 (9) (2002) 821-841, doi:10.1002/fld.331.

[5] W. Rider, L. Margolin, From numerical analysis to implicit subgrid turbulence modeling, 16th AIAA Computational Fluid Dynamics Conference 2003-4101, doi:10.2514/6.2003-4101.

[6] L. G. Margolin, W. J. Rider, The design and construction of implicit LES models, International Journal for Numerical Methods in Fluids 47 (10-11) (2005) 1173-1179, ISSN 1097-0363, doi:10.1002/fld.862.

[7] L. G. Margolin, W. J. Rider, F. F. Grinstein, Modeling turbulent flow with implicit LES, Journal of Turbulence 7 (15) (2006) 27, doi: $10.1080 / 14685240500331595$. 
[8] D. Drikakis, J. Rider, High-Resolution Methods for Incompressible and Low-Speed Flows, vol. 1, Springer-Verlag, ISBN 978-3-540-221364, doi:10.1007/b137615, 2004.

[9] F. F. Grinstein, L. G. Margolin, W. J. Rider, Implicit Large Eddy Simulation: Computing Turbulent Fluid Dynamics, Cambridge University Press, ISBN 9781139463058, 2007.

[10] A. Harten, High resolution schemes for hyperbolic conservation laws, Journal of Computational Physics 49 (3) (1983) 357-393, doi: 10.1016/0021-9991(83)90136-5.

[11] A. Harten, High resolution schemes for hyperbolic conservation laws, Journal of Computational Physics 135 (2) (1997) 260-278, doi: 10.1006/jcph.1997.5713.

[12] E. Toro, Riemann Solvers and Numerical Methods for Fluid Dynamics: A Practical Introduction, Springer, 1999.

[13] D. Drikakis, Advances in turbulent flow computations using high-resolution methods, Progress in Aerospace Sciences 39 (67) (2003) 405424, doi:10.1016/S0376-0421(03)00075-7.

[14] H. Eckelmann, The structure of the viscous sublayer and the adjacent wall region in a turbulent channel flow, Journal of Fluid Mechanics 65 (1974) 439-459, doi:10.1017/S0022112074001479.

[15] A. K. M. F. Hussain, W. C. Reynolds, Measurements in fully developed turbulent channel flow, Journal of Fluids Engineering 97 (1975) 568-578, doi:10.1115/1.3448125.

[16] A. V. Johansson, P. H. Alfredsson, On the structure of turbulent channel flow, Journal of Fluid Mechanics 122 (1982) 295-314, doi: 10.1017/S0022112082002225.

[17] T. Wei, W. W. Willmarth, Reynolds-number effects on the structure of a turbulent channel flow, Journal of Fluid Mechanics 204 (1989) 57-95, doi:10.1017/S0022112089001667.

[18] E.-S. Zanoun, F. Durst, H. Nagib, Evaluating the law of the wall in two-dimensional fully developed turbulent channel flows, Physics of Fluids 15 (10) (2003) 3079-3089, doi:10.1063/1.1608010.

[19] A. Kravchenko, P. Moin, On the effect of numerical errors in large eddy simulations of turbulent flows, Journal of Computational Physics 131 (2) (1997) 310-322, doi:10.1006/jcph.1996.5597.

[20] S. T. Bose, P. Moin, D. You, Grid-independent large-eddy simulation using explicit filtering, Physics of Fluids 22 (10) (2010) 1-11, doi: 10.1063/1.3485774.

[21] B. J. Geurts, Elements of direct and large-eddy simulation, Edwards, 2004.

[22] J. Kim, Progress in pipe and channel flow turbulence, 19612011, Journal of Turbulence 13 (45) (2012) 1-19, doi:10.1080/14685248.2012. 726358.

[23] J. Jiménez, Near-wall turbulence, Physics of Fluids 25 (10) (2013) 1-28, doi:10.1063/1.4824988.

[24] J. Jiménez, How linear is wall-bounded turbulence?, Physics of Fluids 25 (11) (2013) 1-20, doi:10.1063/1.4819081.

[25] A. Cimarelli, E. De Angelis, C. M. Casciola, Paths of energy in turbulent channel flows, Journal of Fluid Mechanics 715 (2013) 436-451, doi: $10.1017 / \mathrm{jfm} .2012 .528$.

[26] L. Wei, A. Pollard, Direct numerical simulation of compressible turbulent channel flows using the discontinuous Galerkin method, Computers \& Fluids 47 (1) (2011) 85-100, doi:10.1016/j.compfluid.2011.02.015.

[27] J.-B. Chapelier, M. de la Llave Plata, F. Renac, E. Lamballais, Evaluation of a high-order discontinuous Galerkin method for the DNS of turbulent flows, Computers \& Fluids 95 (2014) 210-226, doi:10.1016/j.compfluid.2014.02.015.

[28] J. Kim, P. Moin, R. Moser, Turbulence statistics in fully developed channel flow at low Reynolds number, Journal of Fluid Mechanics 177 (1987) 133-166, doi:10.1017/S0022112087000892.

[29] N. N. Mansour, J. Kim, P. Moin, Reynolds-stress and dissipation-rate budgets in a turbulent channel flow, Journal of Fluid Mechanics 194 (1988) 15-44, doi:10.1017/S0022112088002885.

[30] R. D. Moser, J. Kim, N. N. Mansour, Direct numerical simulation of turbulent channel flow up to Re $\operatorname{Re}_{\tau}=590$, Physics of Fluids 11 (4) (1999) 943-945, doi:10.1063/1.869966.

[31] J. C. Del Álamo, J. Jiménez, P. Zandonade, R. D. Moser, Scaling of the energy spectra of turbulent channels, Journal of Fluid Mechanics 500 (2004) 135-144, doi:10.1017/S002211200300733X.

[32] H. Abe, H. Kawamura, Y. Matsuo, Surface heat-flux fluctuations in a turbulent channel flow up to $\operatorname{Re}_{\tau}=1020$ with $\operatorname{Pr}=0.025$ and 0.71 , International Journal of Heat and Fluid Flow 25 (3) (2004) 404-419, doi:10.1016/j.ijheatfluidflow.2004.02.010.

[33] S. Hoyas, J. Jiménez, Scaling of the velocity fluctuations in turbulent channels up to $\operatorname{Re}_{\tau}=2003$, Physics of Fluids 18 (1) (2006) $1-4$, doi:10.1063/1.2162185.

[34] J. Jiménez, S. Hoyas, M. P. Simens, Y. Mizuno, Turbulent boundary layers and channels at moderate Reynolds numbers, Journal of Fluid Mechanics 657 (2010) 335-360, doi:10.1017/S0022112010001370.

[35] X. Wu, P. Moin, Transitional and turbulent boundary layer with heat transfer, Physics of Fluids 22 (8) (2010) 1-8, doi:10.1063/1.3475816.

[36] A. W. Vreman, J. G. M. Kuerten, Comparison of direct numerical simulation databases of turbulent channel flow at $R e_{\tau}=180$, Physics of Fluids 26 (1) (2014) 1-21, doi:10.1063/1.4861064.

[37] G. N. Coleman, J. Kim, R. D. Moser, A numerical study of turbulent supersonic isothermal-wall channel flow, Journal of Fluid Mechanics 305 (1995) 159-183, doi:10.1017/S0022112095004587.

[38] Y. Morinishi, S. Tamano, K. Nakabayashi, Direct numerical simulation of compressible turbulent channel flow between adiabatic and isothermal walls, Journal of Fluid Mechanics 502 (2004) 273-308, doi:10.1017/S0022112003007705.

[39] L. Duan, I. Beekman, M. P. Martín, Direct numerical simulation of hypersonic turbulent boundary layers. Part 2. Effect of wall temperature, Journal of Fluid Mechanics 655 (2010) 419-445, doi:10.1017/S0022112010000959.

[40] D. Taieb, G. Ribert, Direct numerical simulation and large-eddy simulation of supersonic channel flow, Journal of Propulsion and Power 29 (5) (2013) 1064-1075, doi:10.2514/1.B34777.

[41] Y. Tsuji, S. Imayama, P. Schlatter, P. H. Alfredsson, A. V. Johansson, I. Marusic, N. Hutchins, J. Monty, Pressure fluctuation in highReynolds-number turbulent boundary layer: results from experiments and DNS, Journal of Turbulence 13 (50) (2012) 1-19, doi:10.1080/ 14685248.2012 .734625$.

[42] J. Philip, R. Baidya, N. Hutchins, J. P. Monty, I. Marusic, Spatial averaging of streamwise and spanwise velocity measurements in wall- 
bounded turbulence using $\vee$ - and ×-probes, Measurement Science and Technology 24 (11) (2013) 115302, doi:10.1088/0957-0233/24/11/ 115302.

[43] S. Ghosh, H. Foysi, R. Friedrich, Compressible turbulent channel and pipe flow: similarities and differences, Journal of Fluid Mechanics 648 (2010) 155-181, doi:10.1017/S0022112009993004.

[44] C. Chin, J. Monty, A. Ooi, Reynolds number effects in DNS of pipe flow and comparison with channels and boundary layers, International Journal of Heat and Fluid Flow 45 (0) (2014) 33-40, doi:10.1016/j.ijheatfluidflow.2013.11.007.

[45] J. P. Monty, M. S. Chong, Turbulent channel flow: comparison of streamwise velocity data from experiments and direct numerical simulation, Journal of Fluid Mechanics 633 (2009) 461-474, doi:10.1017/S0022112009007769.

[46] H. Ng, J. Monty, N. Hutchins, M. Chong, I. Marusic, Comparison of turbulent channel and pipe flows with varying Reynolds number, Experiments in Fluids 51 (5) (2011) 1261-1281, doi:10.1007/s00348-011-1143-x.

[47] S. Stolz, N. A. Adams, An approximate deconvolution procedure for large-eddy simulation, Physics of Fluids 11 (7) (1999) 1699-1701, doi:10.1063/1.869867.

[48] S. Stolz, N. A. Adams, L. Kleiser, An approximate deconvolution model for large-eddy simulation with application to incompressible wall-bounded flows, Physics of Fluids 13 (4) (2001) 997-1015, doi:10.1063/1.1350896.

[49] S. Hickel, N. A. Adams, J. A. Domaradzki, An adaptive local deconvolution method for implicit LES, Journal of Computational Physics 213 (1) (2006) 413-436, doi:10.1016/j.jcp.2005.08.017.

[50] S. Hickel, N. A. Adams, On implicit subgrid-scale modeling in wall-bounded flows, Physics of Fluids 19 (10) (2007) 1-13, doi:10.1063/1. 2773765.

[51] S. Ghosal, An analysis of numerical errors in large-eddy simulations of turbulence, Journal of Computational Physics 125 (1) (1996) 187206, doi:10.1006/jcph.1996.0088.

[52] B. J. Geurts, J. Fröhlich, A framework for predicting accuracy limitations in large-eddy simulation, Physics of Fluids 14 (6) (2002) 41-44, doi: $10.1063 / 1.1480830$.

[53] F. K. Chow, P. Moin, A further study of numerical errors in large-eddy simulations, Journal of Computational Physics 184 (2) (2003) 366-380, doi:10.1016/S0021-9991(02)00020-7.

[54] J. Meyers, B. J. Geurts, M. Baelmans, Database analysis of errors in large-eddy simulation, Physics of Fluids 15 (9) (2003) $2740-2755$, doi:10.1063/1.1597683.

[55] T. S. Lund, H. J. Kaltenbach, Experiments with explicit filtering for LES using a finite-difference method, in: CTR Annual Research Briefs 1995, Center for Turbulence Research, Stanford University and NASA Ames Research Center, Stanford, California, 91-105, URL http://ntrs.nasa.gov/archive/nasa/casi.ntrs.nasa.gov/19960022301.pdf, 1995.

[56] T. S. Lund, On the use of discrete filters for large eddy simulation, Center for Turbulence Research Annual Research Briefs, URL http: //www.stanford.edu/group/ctr/ResBriefs97/lund.pdf, 1997.

[57] J. Gullbrand, Dynamic modeling in large-eddy simulation of turbulent channel flow: Investigation of two-dimensional versus threedimensional test filtering, International Journal of Numerical Methods for Heat \& Fluid Flow 14 (2004) 467-492, doi:10.1108/ 09615530410532259.

[58] T. T. Brandt, Usability of explicit filtering in large eddy simulation with a low-order numerical scheme and different subgrid-scale models, International Journal for Numerical Methods in Fluids 57 (7) (2008) 905-928, doi:10.1002/fld.1658.

[59] M. Klein, An attempt to assess the quality of large eddy simulations in the context of implicit filtering, Flow, Turbulence and Combustion 75 (1-4) (2005) 131-147, doi:10.1007/s10494-005-8581-6.

[60] J. Gullbrand, F. K. Chow, The effect of numerical errors and turbulence models in large-eddy simulations of channel flow, with and without explicit filtering, Journal of Fluid Mechanics 495 (2003) 323-341, doi:10.1017/S0022112003006268.

[61] J. Gullbrand, Grid-independent large-eddy simulation in turbulent channel flow using three-dimensional explicit filtering, Center for Turbulence Research Annual Research Briefs (2003) 331-342URL http://ctr.stanford.edu/ResBriefs03/gullbrand.pdf.

[62] T. J. R. Hughes, A. A. Oberai, L. Mazzei, Large eddy simulation of turbulent channel flows by the variational multiscale method, Physics of Fluids 13 (6) (2001) 1784-1799, doi:10.1063/1.1367868.

[63] Y. Bazilevs, V. Calo, J. Cottrell, T. Hughes, A. Reali, G. Scovazzi, Variational multiscale residual-based turbulence modeling for large eddy simulation of incompressible flows, Computer Methods in Applied Mechanics and Engineering 197 (1-4) (2007) 173 - 201, ISSN 0045-7825, doi:10.1016/j.cma.2007.07.016.

[64] A. Oberai, J. Liu, D. Sondak, T. Hughes, A residual based eddy viscosity model for the large eddy simulation of turbulent flows, Computer Methods in Applied Mechanics and Engineering 282 (0) (2014) 54 - 70, ISSN 0045-7825, doi:10.1016/j.cma.2014.08.014.

[65] U. Piomelli, Wall-layer models for large-eddy simulations, Progress in Aerospace Sciences 44 (6) (2008) 437-446, doi:10.1016/j.paerosci. 2008.06.001.

[66] P. Spalart, W. Jou, M. Strelets, S. Allmaras, Comments of feasibility of LES for wings, and on a hybrid RANS/LES approach, in: International Conference on DNS/LES, 1997.

[67] N. V. Nikitin, F. Nicoud, B. Wasistho, K. D. Squires, P. R. Spalart, An approach to wall modeling in large-eddy simulations, Physics of Fluids 12 (7) (2000) 1629-1632, doi:10.1063/1.870414.

[68] F. Hamba, A hybrid RANS/LES simulation of turbulent channel flow, Theoretical and Computational Fluid Dynamics 16 (5) (2003) 387403, doi:10.1007/s00162-003-0089-x.

[69] F. Hamba, A hybrid RANS/LES simulation of high-Reynolds-number channel flow using additional filtering at the interface, Theoretical and Computational Fluid Dynamics 20 (2) (2006) 89-101, doi:10.1007/s00162-006-0009-y.

[70] U. Piomelli, E. Balaras, H. Pasinato, K. D. Squires, P. R. Spalart, The inner-outer layer interface in large-eddy simulations with wall-layer models, International Journal of Heat and Fluid Flow 24 (4) (2003) 538-550, doi:10.1016/S0142-727X(03)00048-1.

[71] F. Tessicini, L. Temmerman, M. Leschziner, Approximate near-wall treatments based on zonal and hybrid RANS-LES methods for LES at high Reynolds numbers, International Journal of Heat and Fluid Flow 27 (5) (2006) 789-799, doi:10.1016/j.ijheatfluidflow.2006.03.024.

[72] D. Walters, S. Bhushan, M. Alam, D. Thompson, Investigation of a Dynamic Hybrid RANS/LES Modelling Methodology for Finite-Volume CFD Simulations, Flow, Turbulence and Combustion 91 (3) (2013) 643-667, ISSN 1386-6184, doi:10.1007/s10494-013-9481-9. 
[73] L. Temmerman, M. Hadžiabdić, M. Leschziner, K. Hanjalić, A hybrid two-layer URANS-LES approach for large eddy simulation at high Reynolds numbers, International Journal of Heat and Fluid Flow 26 (2) (2005) 173-190, doi:10.1016/j.ijheatfluidflow.2004.07.006.

[74] K. Abe, A hybrid LES/RANS approach using an anisotropy-resolving algebraic turbulence model, International Journal of Heat and Fluid Flow 26 (2) (2005) 204-222, doi:10.1016/j.ijheatfluidflow.2004.08.009.

[75] C. Duprat, G. Balarac, O. Métais, P. M. Congedo, O. Brugière, A wall-layer model for large-eddy simulations of turbulent flows with/out pressure gradient, Physics of Fluids 23 (1) (2011) 1-12, doi:10.1063/1.3529358.

[76] L. Davidson, The PANS model in a zonal hybrid RANS-LES formulation, International Journal of Heat and Fluid Flow 46 (0) (2014) 112-126, doi:10.1016/j.ijheatfluidflow.2014.01.002.

[77] S. T. Bose, P. Moin, A dynamic slip boundary condition for wall-modeled large-eddy simulation, Physics of Fluids 26 (1) (2014) 1-18, doi:10.1063/1.4849535.

[78] Z. Chen, S. Hickel, A. Devesa, J. Berland, N. A. Adams, Wall modeling for implicit large-eddy simulation and immersed-interface methods, Theoretical and Computational Fluid Dynamics 28 (1) (2014) 1-21, doi:10.1007/s00162-012-0286-6.

[79] S. Utyuzhnikov, Towards development of unsteady near-wall interface boundary conditions for turbulence modeling, Computer Physics Communications 185 (11) (2014) 2879-2884, ISSN 0010-4655, doi:10.1016/j.cpc.2014.07.009.

[80] C. Fureby, F. F. Grinstein, Large eddy simulation of high-Reynolds-number free and wall-bounded flows, Journal of Computational Physics 181 (1) (2002) 68-97, doi:10.1006/jcph.2002.7119.

[81] C. Fureby, F. F. Grinstein, Recent progress on MILES for high Reynolds number flows, Journal of Fluids Engineering 124 (2002) 848-861, doi:10.1115/1.1516576.

[82] F. F. Grinstein, C. Fureby, C. R. DeVore, On MILES based on flux-limiting algorithms, International Journal for Numerical Methods in Fluids 47 (10-11) (2005) 1043-1051, doi:10.1002/fld.925.

[83] C. Fureby, Towards the use of large eddy simulation in engineering, Progress in Aerospace Sciences 44 (6) (2008) 381-396, doi:10.1016/j. paerosci.2008.07.003.

[84] D. Drikakis, C. Fureby, F. F. Grinstein, D. Youngs, Simulation of transition and turbulence decay in the Taylor-Green vortex, Journal of Turbulence 8 (20) (2007) 1-12, doi:10.1080/14685240701250289.

[85] J. A. Domaradzki, Large eddy simulations without explicit eddy viscosity models, International Journal of Computational Fluid Dynamics 24 (10) (2010) 435-447, doi:10.1080/10618562.2010.535792.

[86] B. Thornber, A. Mosedale, D. Drikakis, D. Youngs, R. Williams, An improved reconstruction method for compressible flows with low Mach number features, Journal of Computational Physics 227 (10) (2008) 4873-4894, doi:10.1016/j.jcp.2008.01.036.

[87] B. Thornber, D. Drikakis, R. Williams, D. Youngs, On entropy generation and dissipation of kinetic energy in high-resolution shockcapturing schemes, Journal of Computational Physics 227 (10) (2008) 4853-4872, doi:10.1016/j.jcp.2008.01.035.

[88] D. Drikakis, Embedded turbulence model in numerical methods for hyperbolic conservation laws, International Journal for Numerical Methods in Fluids 39 (2002) 763-781, doi:10.1002/fld.328.

[89] M. Hahn, D. Drikakis, Large eddy simulation of compressible turbulence using high-resolution methods, International Journal for Numerical Methods in Fluids 47 (8-9) (2005) 971-977, doi:10.1002/fld.882.

[90] B. Thornber, A. Mosedale, D. Drikakis, On the implicit large eddy simulations of homogeneous decaying turbulence, Journal of Computational Physics 226 (2) (2007) 1902-1929, doi:10.1016/j.jcp.2007.06.030.

[91] M. Hahn, D. Drikakis, Assessment of large-eddy simulation of internal separated flow, Journal of Fluids Engineering, Transactions of the ASME 131 (7) (2009) 0712011-07120115, doi:10.1115/1.3130243.

[92] M. Hahn, D. Drikakis, Implicit large-eddy simulation of swept-wing flow using high-resolution methods, AIAA Journal 47 (3) (2009) 618-630, doi:10.2514/1.37806.

[93] B. Thornber, D. Drikakis, Implicit large eddy simulation of a deep cavity using high-resolution methods, AIAA Journal 46 (10) (2008) 2634-2645, doi:10.2514/1.36856.

[94] D. Drikakis, M. Hahn, A. Mosedale, B. Thornber, Large eddy simulation using high-resolution and high-order methods, Philosophical Transactions of the Royal Society A: Mathematical, Physical and Engineering Sciences 367 (1899) (2009) 2985-2997, doi:10.1098/rsta. 2008.0312.

[95] E. Toro, M. Spruce, W. Speares, Restoration of the contact surface in the HLL-Riemann solver, Shock waves 4 (1) (1994) 25-34, doi: 10.1007/BF01414629.

[96] A. Harten, P. Lax, B. Leer, On upstream differencing and Godunov-type schemes for hyperbolic conservation laws, SIAM Review 25 (1) (1983) 35-61, doi:10.1137/1025002.

[97] B. van Leer, Towards the ultimate conservative difference scheme I. The quest of monotonicity, in: H. Cabannes, R. Temam (Eds.), Proceedings of the Third International Conference on Numerical Methods in Fluid Mechanics, vol. 18 of Lecture Notes in Physics, Springer Berlin Heidelberg, 163-168, doi:10.1007/BFb0118673, 1973.

[98] B. van Leer, Towards the ultimate conservative difference scheme III. Upstream-centered finite-difference schemes for ideal compressible flow, Journal of Computational Physics 23 (3) (1977) 263-275, doi:10.1016/0021-9991(77)90094-8.

[99] J. Zółtak, D. Drikakis, Hybrid upwind methods for the simulation of unsteady shock-wave diffraction over a cylinder, Computer Methods in Applied Mechanics and Engineering 162 (1(4)) (1998) 165-185, doi:10.1016/S0045-7825(97)00342-3.

[100] K. H. Kim, C. Kim, Accurate, efficient and monotonic numerical methods for multi-dimensional compressible flows: Part I: Spatial discretization, Journal of Computational Physics 208 (2) (2005) 527-569, doi:10.1016/j.jcp.2005.02.021.

[101] K. H. Kim, C. Kim, Accurate, efficient and monotonic numerical methods for multidimensional compressible flows: Part II: Multidimensional limiting process, Journal of Computational Physics 208 (2005) 570-615, doi:10.1016/j.jcp.2005.02.022.

[102] G.-S. Jiang, C.-W. Shu, Efficient implementation of weighted ENO schemes, Journal of Computational Physics 126 (1) (1996) 202-228, doi:10.1006/jcph.1996.0130.

[103] D. S. Balsara, C.-W. Shu, Monotonicity preserving weighted essentially non-oscillatory schemes with increasingly high order of accuracy, Journal of Computational Physics 160 (2) (2000) 405-452, doi:10.1006/jcph.2000.6443.

[104] X.-D. Liu, S. Osher, T. Chan, Weighted essentially non-oscillatory schemes, Journal of Computational Physics 115 (1) (1994) 200-212, 
doi:10.1006/jcph.1994.1187.

[105] J. Cheng, C.-W. Shu, Positivity-preserving Lagrangian scheme for multi-material compressible flow, Journal of Computational Physics 257, Part A (0) (2014) 143-168, ISSN 0021-9991, doi:10.1016/j.jcp.2013.09.047.

[106] J. Shi, Y.-T. Zhang, C.-W. Shu, Resolution of high order WENO schemes for complicated flow structures, Journal of Computational Physics 186 (2) (2003) 690-696, ISSN 0021-9991, doi:10.1016/S0021-9991(03)00094-9.

[107] R. Zhang, M. Zhang, C.-W. Shu, On the order of accuracy and numerical performance of two classes of finite volume WENO schemes, Communications in Computational Physics 9 (2011) 807-827, doi:10.4208/cicp.291109.080410s.

[108] X. Zhang, C.-W. Shu, Positivity-preserving high order finite difference WENO schemes for compressible Euler equations, Journal of Computational Physics 231 (5) (2012) 2245-2258, ISSN 0021-9991, doi:10.1016/j.jcp.2011.11.020.

[109] C.-W. Shu, On high-order accurate weighted essentially non-oscillatory and discontinuous Galerkin schemes for compressible turbulence simulations, Philosophical Transactions of the Royal Society A: Mathematical, Physical and Engineering Sciences 371 (1982), doi:10.1098/ rsta.2012.0172.

[110] D. S. Balsara, C. Altmann, C.-D. Munz, M. Dumbser, A sub-cell based indicator for troubled zones in RKDG schemes and a novel class of hybrid RKDG+HWENO schemes, Journal of Computational Physics 226 (1) (2007) 586-620, ISSN 0021-9991, doi:10.1016/j.jcp.2007.04. 032

[111] A. Mosedale, D. Drikakis, Assessment of very high-order of accuracy in LES models, Journal of Fluids Engineering 129 (12) (2007) 1497-1503, doi:10.1115/1.2801374.

[112] P. Tsoutsanis, V. A. Titarev, D. Drikakis, WENO schemes on arbitrary mixed-element unstructured meshes in three space dimensions, Journal of Computational Physics 230 (4) (2011) 1585-1601.

[113] V. A. Titarev, P. Tsoutsanis, D. Drikakis, WENO schemes for mixed-element unstructured meshes, Communications in Computational Physics 8 (3) (2010) 585-609.

[114] P. Tsoutsanis, A. Antoniadis, D. Drikakis, WENO schemes on arbitrary unstructured meshes for laminar, transitional and turbulent flows, Journal of Computational Physics 256 (2014) 254-276.

[115] E. M. Taylor, M. Wu, M. P. Martn, Optimization of nonlinear error for weighted essentially non-oscillatory methods in direct numerical simulations of compressible turbulence, Journal of Computational Physics 223 (1) (2007) 384-397, ISSN 0021-9991, doi:10.1016/j.jcp. 2006.09.010.

[116] S. B. Pope, Turbulent flows, Cambridge University Press, 2000.

[117] M. Terracol, P. Sagaut, C. Basdevant, A multilevel algorithm for large-eddy simulation of turbulent compressible flows, Journal of Computational Physics 167 (2) (2001) 439-474, doi:10.1006/jcph.2000.6687.

[118] C. Fletcher, Computational techniques for fluid dynamics, vol. 2, Springer Verlag, doi:10.1007/978-3-642-58229-5, 1991.

[119] E. Lenormand, P. Sagaut, L. Ta Phuoc, Large eddy simulation of subsonic and supersonic channel flow at moderate Reynolds number, International Journal for Numerical Methods in Fluids 32 (4) (2000) 369-406, doi:10.1002/(SICI)1097-0363(20000229)32:4〈369:: AID-FLD943>3.0.CO;2-6

[120] E. Lenormand, P. Sagaut, L. T. Phuoc, P. Comte, Subgrid-scale models for large-eddy simulations of compressible wall bounded flows, AIAA Journal 38 (8) (2000) 1340-1350, doi:10.2514/2.1133.

[121] P. G. Huang, G. N. Coleman, P. Bradshaw, Compressible turbulent channel flows: DNS results and modelling, Journal of Fluid Mechanics 305 (1995) 185-218, doi:10.1017/S0022112095004599.

[122] V. Deschamps, Simulation numerique de la turbulence inhomogene incompressible dans un ecoulement de canal plan, Tech. Rep. 1988/5, Onera, 29, Avenue de la Division Leclerc, 92320 Chatillon, France, 1988.

[123] C. Brun, M. Petrovan Boiarciuc, M. Haberkorn, P. Comte, Large eddy simulation of compressible channel flow, Theoretical and Computational Fluid Dynamics 22 (3-4) (2008) 189-212, doi:10.1007/s00162-007-0073-y.

[124] J. C. R. Hunt, A. Way, P. Moin, Eddies, stream, and convergence zones in turbulent flows, Center for Turbulence Research Report CTR-S88, Center for turbulence research, Standford University, URL http: //ctr.stanford.edu/Summer/201306111537.pdf, 1988.

[125] Y. Bazilevs, T. Hughes, Weak imposition of Dirichlet boundary conditions in fluid mechanics, Computers \& Fluids 36 (1) (2007) 12 - 26, ISSN 0045-7930, doi:10.1016/j.compfluid.2005.07.012, challenges and Advances in Flow Simulation and Modeling.

[126] Y. Bazilevs, C. Michler, V. Calo, T. Hughes, Weak Dirichlet boundary conditions for wall-bounded turbulent flows, Computer Methods in Applied Mechanics and Engineering 196 (49-52) (2007) 4853 - 4862, ISSN 0045-7825, doi:10.1016/j.cma.2007.06.026.

[127] J. A. Domaradzki, R. W. Metcalfe, R. S. Rogallo, J. J. Riley, Analysis of subgrid-scale eddy viscosity with use of results from direct numerical simulations, Phys. Rev. Lett. 58 (1987) 547-550, doi:10.1103/PhysRevLett.58.547.

[128] J. A. Domaradzki, W. Liu, M. E. Brachet, An analysis of subgridscale interactions in numerically simulated isotropic turbulence, Physics of Fluids A: Fluid Dynamics 5 (7) (1993) 1747-1759, doi:10.1063/1.858850.

[129] J. A. Domaradzki, W. Liu, C. Hrtel, L. Kleiser, Energy transfer in numerically simulated wallbounded turbulent flows, Physics of Fluids 6 (4) (1994) 1583-1599, doi:10.1063/1.868272.

[130] A. J. Young, W. D. McComb, Effective viscosity due to local turbulence interactions near the cutoff wavenumber in a constrained numerical simulation, Journal of Physics A: Mathematical and General 33 (13), doi:10.1088/0305-4470/33/13/103.

[131] M. Oliveira, J. Su, P. Xie, C. Liu, Truncation error, dissipation and dispersion terms of fifth order WENO and of WCS for 1D conservation law, International Journal of Computer Mathematics 87 (2) (2010) 339-352, doi:10.1080/00207160802036890.

[132] W. J. Rider, The relationship of MPDATA to other high-resolution methods, International Journal for Numerical Methods in Fluids 50 (10) (2006) 1145-1158, ISSN 1097-0363, doi:10.1002/fld.1084. 